JOURNAL OF THE

AMERICAN MATHEMATICAL SOCIETY

Volume 23, Number 4, October 2010, Pages 967-1012

S 0894-0347(10)00667-3

Article electronically published on April 21, 2010

\title{
INTEGRAL MODELS FOR SHIMURA VARIETIES OF ABELIAN TYPE
}

\author{
MARK KISIN
}

\section{Contents}

Introduction 967

1. Reductive groups and $p$-divisible groups $\quad 971$

\begin{tabular}{l|l|} 
(1.1) Cocharacters and filtrations & 971 \\
\hline
\end{tabular}

(1.2) Review of S-modules $\quad 973$

(1.3) Reductive groups and crystalline representations

(1.4) Application to $p$-divisible groups $\quad 978$

(1.5) Deformation theory $\quad 981$

2. Integral canonical models of Hodge type $\quad 986$

\begin{tabular}{l|l} 
(2.1) Shimura varieties & 986
\end{tabular}

(2.2) Absolute Hodge cycles $\quad 989$

\begin{tabular}{ll|} 
(2.3) Integral models & 992 \\
\hline
\end{tabular}

3. Integral canonical models of abelian type $\quad 995$

(3.1) Twisting abelian varieties $\quad 995$

(3.2) The action of $G^{\text {ad }}(\mathbb{Q})$

(3.3) Connected Shimura varieties 1001

(3.4) Integral models II 1005

References

1011

\section{INTRODUCTION}

The problem of constructing smooth models of Shimura varieties was raised by Langlands in his Jugendtraum paper La as a (very small) part of his program to describe the zeta function of a Shimura variety in terms of automorphic $L$ functions. The point of this paper is to show the existence of reasonable models for many Shimura varieties, namely for those which are of abelian type.

To explain some of the properties these integral models ought to satisfy, consider a Shimura datum $(G, X)$ and a Shimura variety $\operatorname{Sh}_{K}(G, X)$ attached to a compact open subgroup $K \subset G\left(\mathbb{A}_{f}\right)$. We will assume that $K$ has the form $K_{p} K^{p}$, where $K_{p} \subset$ $G\left(\mathbb{Q}_{p}\right), K^{p} \subset G\left(\mathbb{A}_{f}^{p}\right)$, and $\mathbb{A}_{f}^{p}$ denotes the finite adeles with trivial $p$-component. By results of Shimura, Deligne, Borovoi and Milne it is known that $\operatorname{Sh}_{K}(G, X)$ has a model over a number field $E=E(G, X) \subset \mathbb{C}$. Let $\mathcal{O}$ be the ring of integers of $E$

Received by the editors November 4, 2008 and, in revised form, December 16, 2009.

2010 Mathematics Subject Classification. Primary 11G18; Secondary 14G35.

The author was partially supported by NSF grant DMS-0017749000. 
and $v \mid p$ a prime of $\mathcal{O}$. Langlands suggested that when $K_{p}$ is hyperspecial [Ti, 3.8], the tower of $E$-schemes

$$
\operatorname{Sh}_{K_{p}}(G, X)=\lim _{K^{p}} \operatorname{Sh}_{K_{p} K^{p}}(G, X)
$$

ought to have a $G\left(\mathbb{A}_{f}^{p}\right)$-equivariant extension to a tower of smooth $\mathcal{O}_{(v) \text {-schemes }}$ $\mathscr{S}_{K_{p}}(G, X)$. The condition on $K_{p}$ means that $K_{p}=G_{\mathbb{Z}_{p}}\left(\mathbb{Z}_{p}\right)$ for a reductive group $G_{\mathbb{Z}_{p}}$ over $\mathbb{Z}_{p}$ with generic fibre $G$. The example of Siegel modular varieties $(G=$ GSp) already shows that, in general, it is not reasonable to ask for a smooth model if $K_{p}$ is merely maximal compact. Hyperspecial subgroups exist if and only if $G$ is quasi-split at $p$ and split over an unramified extension of $\mathbb{Q}_{p}$.

Without a further condition the existence of $\mathscr{S}_{K_{p}}(G, X)$ is formally vacuous, since one could take $\mathscr{S}_{K_{p}}(G, X)=\operatorname{Sh}_{K_{p}}(G, X)$. If the varieties $\operatorname{Sh}_{K}(G, X)$ are proper one can ask that $\mathscr{S}_{K_{p}}(G, X)$ be proper. In general, Milne [Mi 1] suggested that $\mathscr{S}_{K_{p}}(G, X)$ should satisfy a certain extension property. One of the consequences of this property is that the pro-scheme $\mathscr{S}_{K_{p}}(G, X)$ satisfies the valuative criterion for properness for discrete valuation rings of mixed characteristic. In those cases when one imagines that $\mathscr{S}_{K_{p}}(G, X)$ should be a moduli space for abelian varieties, this is justified by the Néron-Ogg-Shafarevich criterion for good reduction of an abelian variety. In fact the extension property formulated by Milne is stronger and has the added benefit of characterizing the pro-scheme $\mathscr{S}_{K_{p}}(G, X)$. We refer to $\$ 2.3$ for a precise formulation. Milne termed a smooth $G\left(\mathbb{A}_{f}^{p}\right)$-equivariant model of $\operatorname{Sh}_{K_{p}}(G, X)$ over $\mathcal{O}_{(v)}$, satisfying the extension property, an integral canonical model.

To state our main theorem we recall that a Shimura datum is said to be of Hodge type if there is an embedding $(G, X) \hookrightarrow\left(\mathrm{GSp}, S^{ \pm}\right)$into the Shimura datum for the symplectic group. Concretely, this means that $\operatorname{Sh}_{K}(G, X)$ has an interpretation as a moduli space for abelian varieties equipped with certain Hodge cycles. A datum $(G, X)$ is said to be of abelian type if there is a datum of Hodge type $\left(G_{2}, X_{2}\right)$ and a central isogeny $G_{2}^{\text {der }} \rightarrow G^{\text {der }}$ which induces an isomorphism $\left(G_{2}^{\text {ad }}, X_{2}^{\text {ad }}\right) \stackrel{\sim}{\longrightarrow}$ $\left(G^{\mathrm{ad}}, X^{\mathrm{ad}}\right)$. Then we prove the following 1

Theorem. Suppose that $(G, X)$ is of abelian type and $K_{p} \subset G\left(\mathbb{Q}_{p}\right)$ hyperspecial. Let $v \mid p$ be a prime of the ring of integers $\mathcal{O} \subset E(G, X)$. If $p>2$, then the proscheme $\operatorname{Sh}_{K_{p}}(G, X)$ admits an extension to a $G\left(\mathbb{A}_{f}^{p}\right)$-equivariant system of smooth $\mathcal{O}_{(v)}$-schemes

$$
\mathscr{S}_{K_{p}}(G, X)=\lim _{K^{p}} \mathscr{S}_{K_{p} K^{p}}(G, X)
$$

which has the extension property.

When $p=2$ we can still prove the theorem under some restrictions on $(G, X)$. We refer the reader to the text for a precise statement in this case.

Our construction also shows that $\mathscr{S}_{K}(G, X)$ carries a vector bundle with an integrable connection, equipped with a family of Hodge tensors. Using this one can obtain natural integral models of the automorphic vector bundles on $\operatorname{Sh}_{K}(G, X)$ (see Mi 2]). This ought to be useful in studying the arithmetic properties of algebraic automorphic forms on $G$.

An important class of Shimura varieties for applications consists of those of PEL type. For these Shimura varieties, integral canonical models were constructed by Kottwitz [Ko, who moreover carried out a large part of Langlands' program on

\footnotetext{
${ }^{1}$ Note that the roles of $G$ and $G_{2}$ are reversed in $\S 3.4$ below.
} 
mod $p$ points in this case. Let us also remark that a proof in the case of abelian type has been claimed by Vasiu. See [Va 1], Va 2, as well as the more recent Va 4, Va 5.

We now describe some of the techniques of this paper in more detail. For an overview, the reader may also wish to consult Ki 4, where we announced these results and sketched some of the arguments. Unsurprisingly, the theorem is first proved for Shimura varieties of Hodge type, and the case of abelian type is deduced from this. (The restrictions when $p=2$ all arise in the first step.) Suppose that $V$ is a $\mathbb{Q}$-vector space equipped with a symplectic pairing $\psi$. We will write $\mathrm{GSp}=\mathrm{GSp}(V, \psi)$. For a Shimura datum of Hodge type, a symplectic embedding $(G, X) \hookrightarrow\left(\mathrm{GSp}, S^{ \pm}\right)$induces an embedding of Shimura varieties

$$
\operatorname{Sh}_{K}(G, X) \hookrightarrow \operatorname{Sh}_{K^{\prime}}\left(\mathrm{GSp}, S^{ \pm}\right)
$$

where $K^{\prime}=K_{p}^{\prime} K^{\prime p} \subset \operatorname{GSp}\left(\mathbb{A}_{f}\right)$ is a compact open subgroup which can be chosen so that $K_{p}^{\prime}$ is maximal compact. The interpretation of $\mathrm{Sh}_{K^{\prime}}\left(\mathrm{GSp}, S^{ \pm}\right)$as a moduli space for abelian varieties leads to a natural integral model $\mathscr{S}_{K^{\prime}}\left(\mathrm{GSp}, S^{ \pm}\right)$. A natural candidate for $\mathscr{S}_{K}(G, X)$ is the normalization of $\mathscr{S}_{K}^{-}(G, X)$, the closure of $\operatorname{Sh}_{K}(G, X)$ in $\mathscr{S}_{K^{\prime}}\left(\mathrm{GSp}, S^{ \pm}\right)$. The pro-scheme $\mathscr{S}_{K_{p}}(G, X)$ is then obviously $G\left(\mathbb{A}_{f}^{p}\right)$ equivariant, and the extension property follows from the corresponding property for $\mathscr{S}_{K_{p}^{\prime}}\left(\mathrm{GSp}, S^{ \pm}\right)$. It remains to show that these schemes are smooth.

This construction is considered in the papers of both Moonen $\mathrm{Mo}$ and Vasiu [Va 1, and in fact goes back to Milne's paper [Mi 1, 2.15]. To try to address the remaining problem of smoothness, Moonen and Vasiu propose relating the complete local ring at a closed characteristic $p$ point of $\mathscr{S}_{K}(G, X)$ to a deformation ring for $p$-divisible groups equipped with a collection of Tate cycles, introduced by Faltings $[\mathrm{Fa}, \S 7] 2$

To apply Faltings' construction one needs a kind of crystalline realization of the group $G$. To explain how we obtain this crystalline version of $G$, let $k$ be a perfect field, $K$ a finite, totally ramified extension of $K_{0}=W(k)[1 / p]$ and $\mathscr{G}$ a $p$-divisible group over $\mathcal{O}_{K}$. Let $L=T_{p} \mathscr{G}^{*}$ be the Tate module of the Cartier dual $\mathscr{G}^{*}$ and $\mathbb{D}$ the Dieudonné module of $\mathscr{G}$. Suppose that $G_{\mathbb{Z}_{p}} \subset \mathrm{GL}(L)$ is a reductive group defined by a finite collection of Galois invariant tensors $\left(s_{\alpha}\right) \subset L^{\otimes}$ (see (1.3) below for the precise definition of a tensor). The $p$-adic comparison isomorphism

$$
L \otimes_{\mathbb{Z}_{p}} B_{\text {cris }} \stackrel{\sim}{\longrightarrow} \mathbb{D} \otimes_{W(k)} B_{\text {cris }}
$$

allows us to think of the $s_{\alpha}$ as being Frobenius invariant tensors in $\operatorname{Fil}^{0} \mathbb{D}[1 / p]^{\otimes}$. The key which allows us to apply Faltings' construction is the following.

\footnotetext{
${ }^{2}$ Of course Vasiu's papers claim to carry out this proposal using his theory of well positioned tensors. Using the notation above, let $\left(t_{\alpha}\right) \in L[1 / p]^{\otimes}$ be a family of tensors fixed by $G$ and containing a perfect alternating pairing on $L[1 / p]$. The family is said to be well positioned if for any faithfully flat $\mathbb{Z}_{p}$-algebra $R$, and any finite free $R$-module $M$ equipped with an isomorphism $M[1 / p] \stackrel{\sim}{\longrightarrow} L[1 / p] \otimes_{\mathbb{Z}_{p}} R$ such that $\left(t_{\alpha}\right) \subset M^{\otimes}$, the closure of $G \otimes_{\mathbb{Z}_{p}} R$ in $\operatorname{GL}(M)$ is a reductive group scheme. We make no use of this notion in this paper. Thus, for example, Proposition (0.2) is a much more specific statement about the behavior of $G$-structures under the $p$-adic comparison isomorphism. In particular, it does not imply the existence of a family of well positioned tensors.
} 
Proposition (0.2). Suppose that $p>2$ or that $\mathscr{G}^{*}$ is connected. Then

(1) $s_{\alpha} \in \mathbb{D}^{\otimes}$ (not just after inverting $p$ ).

(2) The $s_{\alpha}$ define a reductive subgroup of $G_{W} \subset \mathrm{GL}(\mathbb{D})$.

The proposition is proved using the theory of our previous paper Ki 1. In fact we are able to prove something much more general: If $L$ is a Galois stable lattice in a crystalline representation $V$, then the theory of Ki 1 produces a natural, Frobenius stable lattice $L_{\text {cris }} \subset D_{\text {cris }}(L)$. For any closed, reductive subgroup $G_{\mathbb{Z}_{p}} \subset \operatorname{GL}(L)$ defined by a collection of Galois invariant tensors, there is a corresponding reductive subgroup $G_{W} \subset \operatorname{GL}\left(L_{\text {cris }}\right)$. The restriction in the proposition when $p=2$ arises because we do not know whether $L_{\text {cris }}$ may be identified with $\mathbb{D}$ in this case.

With (0.2) in hand we can consider Faltings' deformation ring. This produces a formally smooth quotient $R_{G}$ of the versal deformation ring $R$ of $\mathscr{G}_{0}=\mathscr{G}_{W_{W(k)}} k$. Roughly speaking, $R_{G}$ carries a $p$-divisible group whose crystal is equipped with Frobenius invariant tensors in degree 0 which deform the $s_{\alpha}$. Faltings shows that $R_{G}$ is versal for such deformations, at least for maps into formally smooth complete local $W(k)$-algebras. Using results related to $(0.2)$ above, we are able to show that there is a version of this result for maps into rings of integers in finite extensions of $W(k)[1 / p]$.

Finally consider a closed point $x \in \mathscr{S}_{K}(G, X)$ in characteristic $p$, and let $\mathcal{A}_{x}$ be the corresponding abelian variety. Applying the above theory to the $p$-divisible group of $\mathcal{A}_{x}$, with the $\left(s_{\alpha}\right)$ a suitable collection of Hodge tensors, allows us to identify the complete local ring at $x$ with $R_{G}$.

To deduce the case of abelian type from the case of Hodge type we follow Deligne's construction of canonical models for these varieties [De 3]. Suppose that $K_{2, p} \subset G_{2}\left(\mathbb{Q}_{p}\right)$ is hyperspecial, and let $\operatorname{Sh}_{K_{p}}(G, X)^{+} \subset \operatorname{Sh}_{K_{p}}(G, X)$ denote the connected component corresponding to $1 \in G\left(\mathbb{A}_{f}\right)$. Then $\operatorname{Sh}_{K_{p}}(G, X)^{+}$depends only on the derived group $G^{\text {der }}$, and one can obtain $\operatorname{Sh}_{K_{p}}(G, X)^{+}$from $\operatorname{Sh}_{K_{2, p}}\left(G_{2}, X_{2}\right)^{+}$by dividing by the action of a finite group $\Delta$. Combining this with the (not completely trivial) combinatorics which govern the connected components of $\operatorname{Sh}_{K_{p}}(G, X)$ one can construct $\operatorname{Sh}_{K}(G, X)$ from $\operatorname{Sh}_{K_{2, p}}\left(G_{2}, X_{2}\right)$.

Moonen [Mo, §3] observed that one can make an analogous construction using the components of $\mathscr{S}_{K_{2, p}}\left(G_{2}, X_{2}\right)$. When $p \nmid|\Delta|$ he used a general result about quotients by groups of prime to $p$ order to show that, if $\mathscr{S}_{K_{2, p}}\left(G_{2}, X_{2}\right)$ consists of smooth schemes, then so does the resulting tower $\mathscr{S}_{K}(G, X)$.

To prove this in general one needs to show that $\Delta$ acts freely on $\mathscr{S}_{K_{2, p}}\left(G_{2}, X_{2}\right)$. The description of the complex points of a Shimura variety as a double quotient shows that, roughly speaking, the elements of $\Delta$ arise from the adelic points of $\operatorname{ker}\left(G_{2}^{\text {der }} \rightarrow G^{\text {der }}\right)$ and from the cokernel of $G_{2}^{\text {der }}(\mathbb{Q}) \rightarrow G^{\text {der }}(\mathbb{Q})$. The action of the latter group is related to the action of $G_{2}^{\text {ad }}(\mathbb{Q})$ on $\operatorname{Sh}\left(G_{2}, X_{2}\right)$ considered by Deligne. We give a moduli theoretic description of the action of $G_{2}^{\text {ad }}(\mathbb{Q})$ which can be "reduced $\bmod p$ ", and using it we are able to show that the action of $\Delta$ is always free.

\section{ACKNOWLEDGMENTS}

It is a pleasure to thank A. Beilinson, O. Bültel, B. Conrad, O. Gabber, R. Kottwitz, B. Moonen, M. Nori and G. Pappas for useful comments regarding various versions of this paper. We also thank the referee for a careful and perceptive reading of the paper. 


\section{Reductive Groups AND $p$-Divisible Groups}

(1.1) Cocharacters and filtrations. A connected reductive group scheme $G$ over $S$ is a flat $S$-group scheme whose fibres are geometrically connected and reductive. (This is what is called simply a reductive group scheme in DG.

By a reductive group over a base $S$ we shall mean a flat $S$-group $G$ together with a closed, connected reductive subgroup $G^{0} \subset G$ such that $G / G^{0}$ is finite étale. We call $G^{0}$ the connected component of the identity of $G$. We will use this more general notion in $\S \S 1.3-1.4$ below; however, the main applications of this paper will be concerned with connected reductive groups.

In this subsection we study the condition that a filtration on a representation of a connected reductive group $G$ corresponds to a parabolic subgroup of $G$.

Lemma (1.1.1). Let $S=\operatorname{Spec} A$ be an affine scheme, and $M$ a finite free $A$ module with a decreasing, finite length filtration $M^{\bullet}$, such that $\mathrm{gr}^{\bullet} M$ is finite flat over $A$.

Let $G \subset \mathrm{GL}(M)$ be a closed, connected reductive subgroup over $S$. Denote by $P \subset G$ the closed subgroup which respects the filtration $M^{\bullet}$ and $U \subset P$ the closed subgroup which acts trivially on $\mathrm{gr} \bullet M$.

Then the following conditions are equivalent.

(1) The filtration $M^{\bullet}$ admits a splitting such that the corresponding cocharacter $\mu: \mathbb{G}_{m} \rightarrow \mathrm{GL}(M)$ factors through $G$.

(2) $P \subset G$ is a parabolic subgroup, $U \subset P$ is its unipotent radical, and the grading on $\mathrm{gr} \bullet M$ is induced by a cocharacter $\nu: \mathbb{G}_{m} \rightarrow P / U$.

Proof. (1) $\Longrightarrow$ (2): Note that $\mu$ factors through $P$. Hence once we know that $P$ and $U$ are flat, then we may take $\nu$ to be the composite $\mathbb{G}_{m} \stackrel{\mu}{\rightarrow} P \rightarrow P / U$.

Thus it suffices to prove that $P$ is parabolic with unipotent radical $U$. For this we may work locally in the étale topology of $S$, and assume that $G$ admits a split maximal torus $T \stackrel{\sim}{\longrightarrow} \mathbb{G}_{m}^{r}$ which contains the image of $\mu$. Let $\mathfrak{t}$ and $\mathfrak{g}$ denote the Lie algebras of $T$ and $G$ respectively, and consider the decomposition

$$
\mathfrak{g}=\mathfrak{t}+\bigoplus_{\alpha \in \Phi} \mathfrak{g}^{\alpha}
$$

of $\mathfrak{g}$ into root spaces under the adjoint action of $T$ [DG, I, 4.7.3]. Let $\Phi^{\prime} \subset \Phi$ (resp. $\left.\Phi^{\prime \prime} \subset \Phi\right)$ be the set of roots $\alpha$ such that $\alpha \circ \mu$ is a non-negative (resp. positive) power of the tautological character of $\mathbb{G}_{m}$. Set $\mathfrak{p}=\mathfrak{t}+\bigoplus_{\alpha \in \Phi^{\prime}} \mathfrak{g}^{\alpha}$ and $\mathfrak{u}=\bigoplus_{\alpha \in \Phi^{\prime \prime}} \mathfrak{g}^{\alpha}$.

By [DG, XXVI, 1.4] $\mathfrak{p}$ is the Lie algebra of a parabolic subgroup $P^{\prime} \subset G\left(\Phi^{\prime}\right.$ satisfies the condition (iii) of loc. cit). The action of $\mathfrak{p}$ on $M$ respects the filtration, so we have $P^{\prime} \subset P$. Since $P^{\prime}$ is flat over $S$, to show that $P^{\prime}$ is open in $P$ it suffices to check fibre by fibre, so we may assume that $S=k$, an algebraically closed field. Then an element of $s \in \mathfrak{g}$ acts on $M$ respecting the filtration if and only if it is contained in $\mathfrak{p}$. Hence Lie $P=\mathfrak{p}$. To see this write $s=\sum_{\beta \in \mathbb{Z}} s_{\beta}$, where $\mu$ has weight $\beta$ on $s_{\beta} \in \mathfrak{g}$. If $\beta_{0}$ is the smallest $\beta$ such that $s_{\beta} \neq 0$, choose $m \in M$ of some weight $j$ under the action on $\mu$, and such that $s_{\beta_{0}} m \neq 0$. Then

$$
\mu(z) \cdot s m=(\operatorname{ad} \mu(z))(s) \mu(z) m=\sum_{\beta \in \mathbb{Z}} z^{\beta+j} s_{\beta} m .
$$

Hence the component of $s m$ in degree $\beta_{0}+j$ is $s_{\beta_{0}} m \neq 0$, and $s$ preserves the filtration if and only if $\beta_{0} \geq 0$. 
It follows that $P^{\prime}$ is open in $P$. As $P^{\prime}$ has connected fibres, this implies that $P^{\prime}$ is normal in $P$, and hence that $P=P^{\prime}$. Similarly $\mathfrak{u}$ is the Lie algebra of a unipotent subgroup $U^{\prime} \subset G$ by [DG, XXII 5.11.3] and an argument similar to that above shows that $U^{\prime}$ is open in $U$. However, the fibres of $U$ are clearly unipotent, so we have $U=U^{\prime}$.

$(2) \Longrightarrow(1)$. The group $P / U$ is connected reductive, and since $S$ is affine, there exists a Levi subgroup $L \subset P$, which maps isomorphically to $P / U$ [DG, XXVI, 2.3]. Hence we may take $\mu$ to be $\nu$ composed with the inclusion $P / U \stackrel{\sim}{\longrightarrow} L \subset P$.

(1.1.2) If $M$ and $M^{\bullet}$ are as in (1.1.1) and $G \hookrightarrow \mathrm{GL}(M)$ is a connected reductive subgroup, then we say that a filtration $M^{\bullet}$ is $G$-split if it satisfies the equivalent conditions of (1.1.1). More generally, if $G \hookrightarrow \mathrm{GL}(M)$ is a reductive subgroup, we say that $M^{\bullet}$ is $G$-split if it is $G^{0}$-split, where $G^{0} \subset G$ denotes the connected component of the identity.

When the above embedding arises from an $A$-linear fibre functor on a Tannakian category, the lemma is closely related to [Sa, IV, 2.2.5].

Lemma (1.1.3). With the notation of (1.1.1), suppose that $A$ is a field of characteristic 0 . Then $M^{\bullet}$ is $G$-split if and only if $\langle M\rangle^{\otimes}$, the Tannakian category of $G$-representations generated by $M$, admits a filtration which induces the given filtration on $M$.

Proof. We refer to [Sa, IV §2] for the notion of a filtration on a Tannakian category. If $(1.1 .1)(1)$ holds, then $\mu$ even equips $\langle M\rangle^{\otimes}$ with a grading. Conversely, a filtration on $\langle M\rangle^{\otimes}$ admits a splitting by a theorem of Deligne [Sa, IV, §2.4]. If such a filtration restricts to the given filtration on $M$, then the splitting gives the required cocharacter $\mu$.

Proposition (1.1.4). Suppose that $A$ is a discrete valuation ring with field of fractions $K$ and that $G \hookrightarrow \mathrm{GL}(M)$ and $M^{\bullet}$ are as above.

Then $M^{\bullet}$ is G-split if and only if the induced filtration on $M \otimes_{A} K$ is $\left.G\right|_{K}$-split.

Proof. If $M^{\bullet}$ is $G$-split, then so is the filtration $\left(M \otimes_{A} K\right)^{\bullet}$ on $M \otimes_{A} K$. We prove the converse.

Let $P_{K}$ be the subgroup of $G_{K}$ which respects the filtration on $M \otimes_{A} K$, and $U_{K} \subset P_{K}$ the subgroup which acts trivially on $\operatorname{gr}^{\bullet} M \otimes_{A} K$. If $\left(M \otimes_{A} K\right)^{\bullet}$ is $\left.G\right|_{K^{-}}$ split, then $P_{K}$ is parabolic in $\left.G\right|_{K}, U_{K} \subset P_{K}$ is the unipotent radical of $P_{K}$ and the action of $P_{K} / U_{K}$ on $\operatorname{gr}^{\bullet} M \otimes_{A} K$ is given by a cocharacter $\nu_{K}: \mathbb{G}_{m} \rightarrow P_{K} / U_{K}$.

By [DG, XXVI, 3.5] $P_{K}$ extends uniquely to a parabolic subgroup $P^{\prime} \subset G$. The unipotent radical of $P^{\prime}$ is a smooth subgroup $U^{\prime}$ extending $U_{K}$. Since $P_{K}$ and $U_{K}$ are dense in $P^{\prime}$ and $U^{\prime}$, respectively, $P^{\prime}$ leaves stable the filtration on $M$ and $U^{\prime}$ acts trivially on $\mathrm{gr}^{\bullet} M$.

The cocharacter $\nu: \mathbb{G}_{m} \rightarrow \mathrm{GL}(\mathrm{gr} \bullet M)$ factors through $P^{\prime} / U^{\prime}$ since this is true for its restriction to $K$. As in the proof of (1.1.1), we may identify $P^{\prime} / U^{\prime}$ with a Levi subgroup of $P^{\prime}$ and lift this cocharacter to a cocharacter $\mu: \mathbb{G}_{m} \rightarrow P^{\prime}$ which defines a splitting of the filtration on $M^{\bullet}$. This shows that $M^{\bullet}$ is $G$-split (and hence that $P^{\prime}=P$ and $\left.U^{\prime}=U\right)$.

Proposition (1.1.5). Let $S=\operatorname{Spec} A$ be an affine scheme, $M$ a finite free $A$ module and $G \subset \mathrm{GL}(M)$ a closed, connected reductive subgroup. For any A-algebra $B$, let $\mathrm{G}-\mathrm{Fil}(B)$ denote the set of $G_{B}=G \otimes_{A} B$-split filtrations on $M_{B}=M_{A} \otimes_{A} B$. Then the functor $B \mapsto \mathrm{G}-\mathrm{Fil}(B)$ is representable by a smooth proper $A$-scheme. 
Proof. Let Par denote the functor which assigns to an $A$-algebra $B$ the set of parabolic subgroups $P_{B} \subset G_{B}$. Denote by $\widehat{P a r}$ the functor which assigns to $B$ the set of pairs $\left(P_{B}, \nu\right)$, where $P_{B} \subset G_{B}$ is a parabolic subgroup with unipotent radical $U_{B}$, and $\nu: \mathbb{G}_{m} \rightarrow P_{B} / U_{B}$ is a cocharacter. Then Par is smooth and representable over $A$ [DG, XVI, 3.5] and the morphism $\widetilde{\mathrm{Par}} \rightarrow$ Par is smooth and relatively representable by [DG, XI, 4.2]. Hence $\widetilde{\text { Par }}$ is smooth and representable.

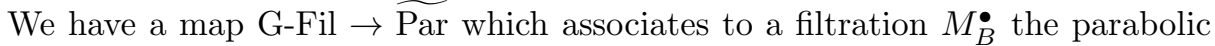
respecting the filtration, together with the cocharacter giving the grading on $\mathrm{gr}^{\bullet} M_{B}$. We will show that G-Fil is represented by a closed and open subscheme of Par. The properness then follows from (1.1.4).

Consider an $A$-algebra $B$, and a parabolic $P_{B}$ of $G_{B}$ with unipotent radical $U_{B}$, together with a cocharacter $\nu: \mathbb{G}_{m} \rightarrow P_{B} / U_{B}$. Fix a Levi subgroup $L_{B} \subset P_{B}$ mapping isomorphically to $P_{B} / U_{B}$, and let $\mu: \mathbb{G}_{m} \rightarrow L_{B}$ be the cocharacter induced by $\nu$. Let $\mathfrak{u}=\operatorname{Lie} U_{B}, \mathfrak{p}=\operatorname{Lie} P_{B}$ and decompose $\mathfrak{u}=\bigoplus_{n \in \mathbb{Z}} \mathfrak{u}^{n}$ and $\mathfrak{p}=\bigoplus_{n \in \mathbb{Z}} \mathfrak{p}^{n}$ under the adjoint action of $\mu$, so that $\mu$ has weight $n$ on $\mathfrak{u}^{n}$ and $\mathfrak{p}^{n}$. Let G-Fil ${ }^{\prime}(B) \subset \widetilde{\operatorname{Par}}(B)$ consist of those pairs $\left(P_{B}, \nu\right)$ such that $\mathfrak{u}^{n}=0$ for $n \leq 0$ and $\mathfrak{p}^{n}=0$ for $n<0$. Any two choices of $L_{B}$ differ by conjugation by an element of $U_{B}$, so this condition does not depend on the choice of $L_{B}$ and G-Fil' is well defined. It is clearly represented by an open and closed subscheme of $\widetilde{\mathrm{Par}}$.

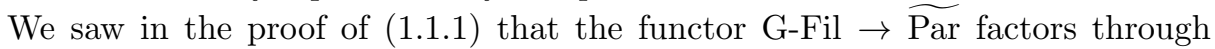
G-Fil'. Conversely, given a point $\left(P_{B}, \nu\right)$ of G-Fil $(B)$ we may choose a Levi subgroup $L_{B} \subset P_{B}$ and consider the filtration given by $\mu$. Let $P_{B}^{\prime}$ and $U_{B}^{\prime}$ denote the parabolic and unipotent subgroups defined by $\mu$ and denote their Lie algebras by $\mathfrak{p}^{\prime}$ and $\mathfrak{u}^{\prime}$, respectively. To see that $P_{B}^{\prime}=P_{B}$, we may work étale locally and assume that $G_{B}$ contains a split maximal torus $T_{B} \subset P_{B}$ with Lie algebra $\mathfrak{t}$ such that $\mu$ factors through $T_{B}$. Decompose $\mathfrak{g}=\mathfrak{t}+\bigoplus_{\alpha \in \Phi} \mathfrak{g}^{\alpha}$ under the adjoint action of $T$. The proof of (1.1.1) shows that $\mathfrak{p} \subset \mathfrak{p}^{\prime}$ and $\mathfrak{u} \subset \mathfrak{u}^{\prime}$. If $\mathfrak{p} \subsetneq \mathfrak{p}^{\prime}$, there is a root space $\mathfrak{g}^{\alpha} \subset \mathfrak{p}^{\prime}$ which is not contained in $\mathfrak{p}$. But then $\mathfrak{g}^{-\alpha} \subset \mathfrak{u}$ [DG, XXII, 5.11.3] and so $\mathfrak{g}^{-\alpha} \subset \mathfrak{u}^{\prime}$ whence $\mathfrak{g}^{\alpha} \nsubseteq \mathfrak{p}^{\prime}$, a contradiction.

It follows that $P_{B}^{\prime}=P_{B}$ and $U_{B}^{\prime}=U_{B}$. In particular these groups are independent of the choice of $L_{B}$, and the filtration depends only on $\nu$ and not on $\mu$. This gives a map G-Fil ${ }^{\prime} \rightarrow$ G-Fil which is a section to the inclusion G-Fil $\rightarrow$ G-Fil'. Hence G-Fil $=$ G-Fil' is open and closed in $\widetilde{\text { Par. }}$.

(1.1.6) In the situation of (1.1.1) let $\mu: \mathbb{G}_{m} \rightarrow G$ be a cocharacter inducing the given filtration $M^{\bullet}$. The centralizer $L$ of $\mu$ in $G$ is a Levi subgroup with Lie algebra $\mathfrak{t}+\bigoplus_{\alpha \in \Phi^{\prime} \backslash \Phi^{\prime \prime}} \mathfrak{g}_{\alpha}$ [DG, XXVI, 5.11.3]. The parabolic subgroup $P^{\prime}$ defined by $\mu^{-1}$ satisfies $P^{\prime} \cap P=L$. We will refer to the unipotent radical $U^{\prime}$ of $P^{\prime}$ as the opposite unipotent defined by $\mu$. We have Lie $G=\operatorname{Lie} P \oplus \operatorname{Lie} U^{\prime}$ [DG, XXVI, 4.3.2].

(1.2) Review of S-modules. We now recall some of the results of [Ki 1] regarding the classification of crystalline representations and $p$-divisible groups.

Let $k$ be a perfect field of characteristic $p, W=W(k)$ its ring of Witt vectors and $K_{0}=W(k)[1 / p]$. Let $K$ be a finite totally ramified extension of $K_{0}$, and $\mathcal{O}_{K}$ its ring of integers. Fix an algebraic closure $\bar{K}$ of $K$, and set $G_{K}=\operatorname{Gal}(\bar{K} / K)$.

We denote by $\operatorname{Rep}_{G_{K}}^{\text {cris }}$ the category of crystalline $G_{K}$-representations, and by $\operatorname{Rep}_{G_{K}}^{\text {criso }}$ the category of $G_{K}$-stable $\mathbb{Z}_{p}$-lattices spanning a representation in $\operatorname{Rep}_{G_{K}}^{\text {cris }}$. 
For $V$ a crystalline representation, recall Fontaine's functors

$$
D_{\text {cris }}(V)=\left(B_{\text {cris }} \otimes_{\mathbb{Q}_{p}} V\right)^{G_{K}} \quad \text { and } \quad D_{\mathrm{dR}}(V)=\left(B_{\mathrm{dR}} \otimes_{\mathbb{Q}_{p}} V\right)^{G_{K}} .
$$

Fix a uniformiser $\pi \in K$, and let $E(u) \in W(k)[u]$ be the Eisenstein polynomial for $\pi$. We set $\mathfrak{S}=W \llbracket u \rrbracket$ equipped with a Frobenius $\varphi$ which acts as the usual Frobenius on $W$ and sends $u$ to $u^{p}$.

Let $\operatorname{Mod}_{/ \mathfrak{S}}^{\varphi}$ denote the category of finite free $\mathfrak{S}$-modules $\mathfrak{M}$ equipped with a Frobenius semi-linear isomorphism 3

$$
1 \otimes \varphi: \varphi^{*}(\mathfrak{M})[1 / E(u)] \stackrel{\sim}{\longrightarrow} \mathfrak{M}[1 / E(u)] .
$$

Note that this definition differs slightly from that of [Ki 1], where we insisted that the above map be induced by a map $\varphi^{*}(\mathfrak{M}) \rightarrow \mathfrak{M}$. This is related to the fact that in loc. cit we considered crystalline representations with Hodge-Tate weights $\leq 0$, whereas here we will allow arbitrary Hodge-Tate weights.

For $i \in \mathbb{Z}$ we set

$$
\operatorname{Fil}^{i} \varphi^{*}(\mathfrak{M})=(1 \otimes \varphi)^{-1}\left(E(u)^{i} \mathfrak{M}\right) \cap \varphi^{*}(\mathfrak{M}) .
$$

Let $\mathcal{O}_{\mathcal{E}}$ denote the $p$-adic completion of $\mathfrak{S}_{(p)}$ and denote by $\operatorname{Mod}_{/ \mathcal{O}_{\mathcal{E}}}^{\varphi}$ the category of finite free $\mathcal{O}_{\mathcal{E}}$-modules $M$ equipped with an isomorphism $\varphi^{*}(M) \stackrel{\sim}{\longrightarrow} M$. We have a functor

$$
\operatorname{Mod}_{/ \mathfrak{S}}^{\varphi} \rightarrow \operatorname{Mod}_{/ \mathcal{O}_{\mathcal{E}}}^{\varphi} ; \quad \mathfrak{M} \mapsto \mathcal{O}_{\mathcal{E}} \otimes_{\mathfrak{S}} \mathfrak{M}
$$

Theorem (1.2.1). There exists a fully faithful tensor functor

$$
\mathfrak{M}: \operatorname{Rep}_{G_{K}}^{\text {criso }} \rightarrow \operatorname{Mod}_{/ \mathfrak{S}}^{\varphi},
$$

which is compatible with the formation of symmetric and exterior powers. If $L$ is in $\operatorname{Rep}_{G_{K}}^{\text {criso }}, V=L \otimes_{\mathbb{Z}_{p}} \mathbb{Q}_{p}$, and $\mathfrak{M}=\mathfrak{M}(L)$, then

(1) There are canonical isomorphisms

$$
D_{\text {cris }}(V) \stackrel{\sim}{\longrightarrow} \mathfrak{M} / u \mathfrak{M}[1 / p] \quad \text { and } \quad D_{\mathrm{dR}}(V) \stackrel{\sim}{\longrightarrow} \varphi^{*}(\mathfrak{M}) \otimes_{\mathfrak{S}} K,
$$

where the map $\mathfrak{S} \rightarrow K$ is given by $u \mapsto \pi$. The first isomorphism is compatible with Frobenius, and the second maps $\operatorname{Fil}^{i} \varphi^{*}(\mathfrak{M}) \otimes_{W} K_{0}$ onto $\operatorname{Fil}^{i} D_{\mathrm{dR}}(V)$ for $i \in \mathbb{Z}$.

(2) There is a canonical isomorphism

$$
\mathcal{O}_{\widehat{\mathcal{E}} \text { ur }} \otimes_{\mathbb{Z}_{p}} L \stackrel{\sim}{\longrightarrow} \mathcal{O}_{\widehat{\mathcal{E} u r}} \otimes_{\mathfrak{S}} \mathfrak{M},
$$

where $\mathcal{O}_{\widehat{\mathcal{E}} \text { ur }}$ is a certain faithfully flat and formally étale $\mathcal{O}_{\mathcal{E}}$-algebra.

(3) If $k^{\prime} / k$ is an algebraic extension of fields, then there exists a canonical $\varphi$ equivariant isomorphism

$$
\mathfrak{M}\left(\left.L\right|_{G_{K^{\prime}}}\right) \stackrel{\sim}{\longrightarrow} \mathfrak{M}(L) \otimes_{\mathfrak{S}} \mathfrak{S}^{\prime},
$$

where $\mathfrak{S}^{\prime}=W\left(k^{\prime}\right) \llbracket u \rrbracket$ and $G_{K^{\prime}}=\operatorname{Gal}\left(\bar{K} \cdot W\left(k^{\prime}\right)[1 / p] / K \cdot W\left(k^{\prime}\right)[1 / p]\right)$.

\footnotetext{
${ }^{3}$ We will need to consider various rings equipped with a fixed lift of Frobenius. Such a lift will be denoted simply by $\varphi$, except if we need to consider different lifts of Frobenius on the same ring, in which case this will of course be made clear in the notation. A Frobenius on a module over such a ring is always semi-linear and will be denoted by the same symbol as the Frobenius lift on the ring.
} 
Proof. This is an exercise in assembling the results of [Ki 1]:

Let $\operatorname{Mod}_{/ \mathfrak{S}}^{\varphi,+}$ denote the full subcategory of $\operatorname{Mod}_{/ \mathfrak{S}}^{\varphi}$ such that the map $1 \otimes \varphi$ induces a map $\varphi^{*}(\mathfrak{M}) \rightarrow \mathfrak{M}$. Let $K_{\infty}=K\left(\sqrt[p^{n}]{\pi}\right)_{n \geq 1}$ be the field obtained from $K$ by adjoining a compatible sequence of $p$-power roots of $\pi$. Write $G_{K_{\infty}}=\operatorname{Gal}\left(\bar{K} / K_{\infty}\right)$ and denote by $\operatorname{Rep}_{G_{K_{\infty}}}^{\circ}$ the category of continuous representations of $G_{K_{\infty}}$ on finite free $\mathbb{Z}_{p}$-modules.

Then we have functors

$$
\operatorname{Mod}_{/ \mathfrak{S}}^{\varphi,+} \stackrel{\mathfrak{M} \mapsto \mathcal{O}_{\mathcal{E}} \otimes_{\mathcal{E}} \mathfrak{M}}{\longrightarrow} \operatorname{Mod}_{/ \mathcal{O}_{\mathcal{E}}}^{\varphi} \stackrel{\sim}{\longrightarrow} \operatorname{Rep}_{G_{K_{\infty}}}^{\circ},
$$

where the second functor is the equivalence of $[\mathrm{Fo}$, A.1.2.7], and the first functor is fully faithful by [Ki 1, 2.1.12] (see also Ki 2, E.4]). Denote by $\operatorname{Rep}_{G_{K_{\infty}}}^{\mathrm{fh}, \circ}$ the essential image of the composite of (1.2.2).

Let $\operatorname{Rep}_{\text {cris }}^{-, \circ}$ denote the category of $G_{K}$-stable $\mathbb{Z}_{p}$-lattices in crystalline representations with Hodge-Tate weights $\leq$ 0. By [Ki 1, 2.1.14], the functor

$$
\operatorname{Rep}_{\text {cris }}^{-, \circ} \rightarrow \operatorname{Rep}_{G_{K_{\infty}}}^{\circ} ;\left.\quad L \mapsto L\right|_{G_{K_{\infty}}}
$$

is fully faithful. If $L$ is in $\operatorname{Rep}_{\text {cris }}^{-\circ}$, then [Ki $\left.1,2.1 .5\right]$ implies that $L[1 / p]$ contains a $G_{K_{\infty}}$-stable lattice in $\operatorname{Rep}_{G_{K_{\infty}}}^{\mathrm{fh}, \mathrm{o}}$, and hence $\left.L\right|_{G_{K_{\infty}}}$ itself is in $\operatorname{Rep}_{G_{K_{\infty}}}^{\mathrm{fh}, \circ}$ by Ki 1, 2.1.15].

We define $\mathfrak{M}$ as the composite

$$
\mathfrak{M}: \operatorname{Rep}_{\text {cris }}^{-, \circ} \rightarrow \operatorname{Rep}_{G_{K_{\infty}}}^{\mathrm{fh}, \circ} \underset{(1.2 .2)}{\stackrel{\sim}{\longrightarrow}} \operatorname{Mod}_{/ \mathfrak{S}}^{\varphi,+} .
$$

To extend this to a functor on $\operatorname{Rep}_{G_{K}}^{\text {criso }}$, let $\mathfrak{S}(1)(\operatorname{resp} . \mathfrak{S}(-1))$ in $\operatorname{Mod}_{/ \mathfrak{S}}^{\varphi}$ be the object with underlying $\mathfrak{S}$-module equal to $\mathfrak{S}$ and $\varphi$ given by sending 1 to $E(0) / p E(u)$ (resp. $p E(u) / E(0))$. Then $\mathfrak{M}\left(\mathbb{Z}_{p}(-1)\right)=\mathfrak{S}(-1)$, for example by Ki 1 , 2.1.5], and for any $L$ in $\operatorname{Rep}_{\text {cris }}^{\circ}$ we set $\mathfrak{M}(L)=\mathfrak{M}(L(-m)) \otimes \mathfrak{S}(1)^{\otimes m}$ for $m$ a positive integer such that $L(-m)[1 / p]$ has all Hodge-Tate weights $\leq 0$.

(1) now follows from [Ki 1, 2.1.5] and the construction of the quasi-inverse functors $D$ and $\mathcal{M}$ in Ki 1, $\$ 1.2$. (2) follows from the construction of the equivalence in [Fo, A.1]. This also implies the compatibility with symmetric tensors: For $n \geq 1$, there is a natural map

$$
\operatorname{Sym}^{n}(\mathfrak{M}(L)) \rightarrow \mathfrak{M}\left(\operatorname{Sym}^{n}(L)\right) .
$$

Using (2) we see that this map is an isomorphism after tensoring by $\otimes_{\mathfrak{S}} \mathcal{O}_{\mathcal{E}}$. Hence it is an isomorphism by [Ki 1, 2.1.9]. The argument for exterior powers is similar.

Finally (3) is easily seen directly from the construction of the functor $\mathfrak{M}$.

(1.3) Reductive groups and crystalline representations. Let $L$ be a $G_{K^{-}}$ stable lattice in a crystalline representation and let $G \subset \mathrm{GL}(L)$ be a reductive group such that the $G_{K}$-action on $L$ factors through $G\left(\mathbb{Z}_{p}\right)$. We will apply the theory of the previous section to produce a "crystalline realization" of $G$.

(1.3.1) Let $R$ be a Noetherian ring and $M$ a finite free $R$-module. We begin by establishing criteria under which a closed $R$-flat subgroup $G \subset \mathrm{GL}(M)$ can be described as the scheme-theoretic stabilizer of a collection of tensors.

It will be convenient to denote simply by $M^{\otimes}$ the direct sum of all the $R$-modules which can be formed from $M$ using the operations of taking duals, tensor products, symmetric powers and exterior powers. Note that $M^{\otimes} \stackrel{\sim}{\longrightarrow} M^{* \otimes}$ so that a tensor in the left-hand side may be regarded in the right-hand side. 
If $\left(s_{\alpha}\right) \subset M^{\otimes}$, and $G \subset \mathrm{GL}(M)$ is the pointwise stabilizer of the $s_{\alpha}$, we say that $G$ is the group defined by the tensors $s_{\alpha}$.

Proposition (1.3.2). Suppose that $R$ is a discrete valuation ring of mixed characteristic, and let $G \subset \mathrm{GL}(M)$ be a closed $R$-flat subgroup whose generic fiber is reductive. Then $G$ is defined by a finite collection of tensors $\left(s_{\alpha}\right) \subset M^{\otimes}$.

Proof. The proof is similar to that of [De 2, Prop. 3.1].

Let $N$ be an $R$-module equipped with an action of $\mathrm{GL}(M)$ and $N^{\prime} \subset N$ a submodule such that $N^{\prime} / N$ is $R$-flat. If $\left(s_{\alpha}\right) \subset N^{\prime}$, then the subgroup of $\operatorname{GL}(M)$ fixing the $s_{\alpha}$ is the same as the subgroup fixing the $s_{\alpha}$ considered as elements of $N$.

Let $\mathcal{O}_{\mathrm{GL}}$ denote the Hopf algebra of $\mathrm{GL}(M)$. For any finite projective $R$-module $W$ with an action of GL(M), let $W_{0}$ denote $W$ with the trivial GL( $\left.M\right)$-action.

We have the inclusion of $R$-schemes $\mathrm{GL}(M) \subset$ End $(M)$, which is fibre by fibre dense. Thus

$$
\mathcal{O}_{\mathrm{GL}}=\underline{\lim }_{n} \operatorname{Sym}^{\bullet}\left(M \otimes M_{0}^{*}\right) \otimes(\operatorname{det} M)^{-n},
$$

with the transition maps being given by multiplication by $\operatorname{det} \otimes \delta^{-1}$, where $\operatorname{det} \epsilon$ $\operatorname{Sym}^{\bullet}\left(M \otimes M_{0}^{*}\right)$ is the determinant and $\delta \in \operatorname{det} M$ is some fixed basis vector. The transition maps in the direct limit remain injective after any base change $R \rightarrow R^{\prime}$. Hence each term in the limit is a direct summand in the next, and it suffices to find tensors $\left(s_{\alpha}\right) \subset \mathcal{O}_{\mathrm{GL}}$ defining $G$.

For any finite projective $R$-module $W$ with an action of $\mathrm{GL}(M)$, the $\mathcal{O}_{\mathrm{GL}^{-}}$ comodule structure on $W$ gives a $\mathrm{GL}(M)$-equivariant map $W \rightarrow W_{0} \otimes_{R} \mathcal{O}_{\mathrm{GL}}$. This map is injective and its cokernel is a direct summand, a section being induced by the identity section $\mathcal{O}_{\mathrm{GL}} \rightarrow R$. Hence it suffices to find elements defining $G$ in any representation of GL $(M)$ on a finite projective $R$-module.

Now let $I \subset \mathcal{O}_{\mathrm{GL}}$ denote the ideal of $G$. Then $G$ is the scheme-theoretic stabilizer of $I$. Let $W \subset \mathcal{O}_{\mathrm{GL}}$ be a finite rank, GL $(M)$-stable, saturated $R$-submodule such that $W \cap I$ contains a set of generators of $I$. Then $G$ is the stabilizer of $W \cap I \subset W$. If $r=\operatorname{rk}_{R} W \cap I$, then $L=\wedge^{r}(W \cap I) \subset \wedge^{r} W$ is a line, and $G$ is the stabilizer of $L$.

Since $G$ has reductive generic fibre the quotient map $\left(\wedge^{r} W\right)^{*} \rightarrow L^{*}$ has a $G$ equivariant splitting over the generic point $\eta \in \operatorname{Spec} R$. Hence there exists a $G$-stable line $\tilde{L}^{*} \subset\left(\wedge^{r} W\right)^{*}$ which maps isomorphically to $L^{*}$ over $\eta$. Now $G$ acts trivially on $L \otimes_{R} \tilde{L}^{*}$ as this is true over $\eta$, and the stabilizer of $L \otimes_{R} \tilde{L}^{*} \subset\left(\wedge^{r} W\right) \otimes_{R}\left(\wedge^{r} W\right)^{*}$ is equal to $G$.

(1.3.3) Let $L$ be a $G_{K}$-stable lattice in a crystalline representation and $G \subset$ $\operatorname{GL}(L)$ a reductive group. Then by (1.3.2) $G$ is defined by a finite collection of tensors $\left(s_{\alpha}\right) \subset L^{\otimes}$, and the $G_{K}$-action on $L$ factors through $G\left(\mathbb{Z}_{p}\right)$ if and only if these tensors are $G_{K}$-invariant.

If this is the case, we may view the tensors $s_{\alpha}$ as morphisms $s_{\alpha}: \mathbf{1} \rightarrow L^{\otimes}$ in $\operatorname{Rep}_{G_{K}}^{\text {criso }}$. Applying the functor $\mathfrak{M}$ of the theorem, we obtain morphisms $\tilde{s}_{\alpha}: \mathbf{1} \rightarrow$ $\mathfrak{M}(L)^{\otimes}$ in $\operatorname{Mod}_{/ \mathfrak{S}}^{\varphi}$.

Proposition (1.3.4). Let $L$ be in $\operatorname{Rep}_{G_{K}}^{\text {criso }}$ and $G \subset \mathrm{GL}(L)$ a reductive $\mathbb{Z}_{p}$-subgroup defined by a finite collection of $G_{K}$-invariant tensors $\left(s_{\alpha}\right) \subset L^{\otimes}$. If $\mathfrak{M}=\mathfrak{M}(L)$, then $\left(\tilde{s}_{\alpha}\right) \subset \mathfrak{M}^{\otimes}$ defines a reductive subgroup of $\mathrm{GL}(\mathfrak{M})$.

If $k$ is separably closed, then there is an $\mathfrak{S}$-linear isomorphism. $\mathfrak{M} \stackrel{\sim}{\longrightarrow} L \otimes_{\mathbb{Z}_{p}} \mathfrak{S}$ which takes the tensor $\tilde{s}_{\alpha}$ to $s_{\alpha}$. In particular, the subgroup $G_{\mathfrak{S}} \subset \mathrm{GL}(\mathfrak{M})$ defined by $\left(\tilde{s}_{\alpha}\right)$ is isomorphic to $G \times \times_{\operatorname{Spec}} \mathbb{Z}_{p}$ Spec $\mathfrak{S}$. 
Proof. Using the compatibility (1.2.1)(3), it suffices to prove the proposition when $k$ is separably closed. Moreover the second statement implies the first.

Now suppose that $k$ is separably closed, set $\mathfrak{M}^{\prime}=L \otimes_{\mathbb{Z}_{p}} \mathfrak{S}$, and let $P \subset$ $\underline{\operatorname{Hom}}_{\mathfrak{S}}\left(\mathfrak{M}, \mathfrak{M}^{\prime}\right)$ be the subscheme of isomorphisms between $\mathfrak{M}$ and $\mathfrak{M}^{\prime}$ which take $\tilde{s}_{\alpha}$ to $s_{\alpha}$. The fibres of $P$ are either empty or a torsor under $G$. We claim that $P$ is a $G$-torsor. That is, $P$ is flat over $\mathfrak{S}$ with non-empty fibres. The claim implies the proposition since a torsor under a reductive group is étale locally trivial, while the ring $\mathfrak{S}$ is strictly Henselian as $k$ is separably closed, so any $G$ torsor over $\mathfrak{S}$ is trivial.

To prove the claim we proceed in several steps. For $R$ a $\mathfrak{S}$-algebra, we set $P_{R}=P \times_{\operatorname{Spec} \mathfrak{S}} \operatorname{Spec} R$.

Step 1. $P_{\mathfrak{S}_{(p)}}$ is a $G$-torsor. Since $\mathcal{O}_{\widehat{\mathcal{E}} \text { ur }}$ is faithfully flat over $\mathcal{O}_{\mathcal{E}}$ and $\mathcal{O}_{\mathcal{E}}$ is faithfully flat over $\mathfrak{S}_{(p)}$, it suffices to show that $P_{\mathcal{O}_{\overparen{\varepsilon u r}}}$ is a $G$-torsor. However the isomorphism in $(1.2 .1)(2)$ shows that $P_{\mathcal{O}_{\widehat{\mathcal{E}} \text { ur }}}$ is a trivial $G$-torsor.

Step 2. $P_{K_{0}}$ is a $G$-torsor, where we regard $K_{0}$ as a $\mathfrak{S}$-algebra via $u \mapsto 0$. This follows from (1.2.1)(1), which implies the existence of a canonical isomorphism

$$
B_{\mathrm{dR}} \otimes_{\mathbb{Z}_{p}} L \stackrel{\sim}{\longrightarrow} B_{\mathrm{dR}} \otimes_{W} \mathfrak{M} / u \mathfrak{M} .
$$

Step 3. $P_{\mathfrak{S}[1 / p u]}$ is a $G$-torsor. Let $U \subset \operatorname{Spec} \mathfrak{S}[1 / u p]$ denote the maximal open subset over which $P$ is flat with non-empty fibres. By Step 1, we know this subset is non-empty, since it contains the generic point. In particular, the complement of $U$ in Spec $\mathfrak{S}[1 / u p]$ contains finitely many closed points.

Let $x \in \operatorname{Spec} \mathfrak{S}[1 / u p]$ be a closed point. If $x \notin U$, we consider two cases. If $|u(x)|<|\pi|$, then since the $s_{\alpha}$ are Frobenius invariant, we have $P_{\mathfrak{S}[1 / p]} \stackrel{\sim}{\longrightarrow}$ $\varphi^{*}\left(P_{\mathfrak{S}[1 / p]}\right)$ in a formal neighbourhood of $x$. Hence $P_{\mathfrak{S}}[1 / p]$ cannot be a $G$-torsor at $\varphi(x)$, since $\varphi$ is a faithfully flat map on $\mathfrak{S}$. Repeating the argument we find $\varphi(x), \varphi^{2}(x), \ldots \notin U$, which gives a contradiction.

Similarly, if $|u(x)| \geq|\pi|$, consider a sequence of points $x_{0}, x_{1}, \ldots$ with $x_{0}=$ $x$, and $\varphi\left(x_{i+1}\right)=x_{i}$. For $i \geq 1$, we have $P_{\mathfrak{S}[1 / p]} \stackrel{\sim}{\longrightarrow} \varphi^{*}\left(P_{\mathfrak{S}[1 / p]}\right)$ in a formal neighbourhood of $x_{i}$, so we find that $x_{i} \notin U$ for $i \geq 1$.

Step 4. $P_{\mathfrak{S}[1 / p]}$ is a $G$-torsor. By Step 3, it suffices to show that the restriction of $P$ to $K_{0} \llbracket u \rrbracket$ is a $G$-torsor. For any $\mathfrak{N}$ in $\operatorname{Mod}_{/ \mathfrak{S}}^{\varphi}$ there is a unique, $\varphi$-equivariant isomorphism

$$
\mathfrak{N} \otimes_{\mathfrak{S}} K_{0} \llbracket u \rrbracket \stackrel{\sim}{\longrightarrow} K_{0} \llbracket u \rrbracket \otimes_{K_{0}} \mathfrak{N} / u \mathfrak{N}[1 / p]
$$

lifting the identity map on $\mathfrak{N} / u \mathfrak{N} \otimes_{\mathcal{O}_{K_{0}}} K_{0}$, which is functorial in $\mathfrak{N}$ (see, for example, $\mathrm{Ki} 1,1.2 .6])$. Applying this to $\mathfrak{M}$ and the morphisms $\tilde{s}_{\alpha}$ shows that the restriction of $P$ to $K_{0} \llbracket u \rrbracket$ is isomorphic to $P_{K_{0}} \otimes_{K_{0}} K_{0} \llbracket u \rrbracket$, which is a $G$-torsor by Step 2.

Step 5. $P$ is a $G$-torsor. Let $U$ be the complement of the closed point in Spec $\mathfrak{S}$. By Steps 1 and 4 we know that $\left.P\right|_{U}$ is a $G$-torsor. By a result of Colliot-Thélène and Sansuc [CS, Thm. 6.13], $P$ extends to a $G$-torsor over $\mathfrak{S}$ and, as we remarked above, any such torsor is trivial. Hence $\left.P\right|_{U}$ is trivial, and there is an isomorphism $\left.\left.\mathfrak{M}\right|_{U} \stackrel{\sim}{\longrightarrow} \mathfrak{M}^{\prime}\right|_{U}$ taking $\tilde{s}_{\alpha}$ to $s_{\alpha}$. Since any vector bundle over $U$ has a canonical extension to $\mathfrak{S}$, obtained by taking its global sections, this isomorphism extends 
to $\mathfrak{S}$. This implies that $P$ is the trivial $G$-torsor and completes the proof of the proposition.

Corollary (1.3.5). With the assumptions of (1.3.4), suppose that $G$ is connected and $k$ is finite. Then there exists an isomorphism $\mathfrak{M} \stackrel{\sim}{\longrightarrow} L \otimes_{\mathbb{Z}_{p}} \mathfrak{S}$ which takes the tensor $\tilde{s}_{\alpha}$ to $s_{\alpha}$. In particular, the subgroup $G_{\mathfrak{S}} \subset \mathrm{GL}(\mathfrak{M})$ defined by $\left(\tilde{s}_{\alpha}\right)$ is isomorphic to $G \times \times_{\mathrm{Spec}} \mathbb{Z}_{p} \operatorname{Spec} \mathfrak{S}$.

Proof. As in (1.3.4) we set $\mathfrak{M}^{\prime}=L \otimes_{\mathbb{Z}_{p}} \mathfrak{M}$, and we denote by $P \subset \underline{\operatorname{Hom}}_{\mathfrak{S}}\left(\mathfrak{M}, \mathfrak{M}^{\prime}\right)$ the subscheme of isomorphisms between $\mathfrak{M}$ and $\mathfrak{M}^{\prime}$ which take $\tilde{s}_{\alpha}$ to $s_{\alpha}$. Then $P$ is a $G$-torsor by (1.3.4). Since $G$ is connected and $k$ is finite, any such torsor is trivial Sp, 4.4], and the corollary follows.

Corollary (1.3.6). Let $L$ be a $G_{K}$-stable lattice in a crystalline representation $V$, $\mathfrak{M}=\mathfrak{M}(L)$ and $\left(s_{\alpha}\right) \subset L^{\otimes}$ a collection of $G_{K}$-invariant tensors which define a reductive subgroup $G$ of $\mathrm{GL}(L)$. Then:

(1) If we view $\left(s_{\alpha}\right) \subset \mathrm{Fil}^{0} D_{\text {cris }}(V)^{\otimes}$ via the -adic comparison isomorphism

$$
B_{\text {cris }} \otimes_{\mathbb{Z}_{p}} L \stackrel{\sim}{\longrightarrow} B_{\text {cris }} \otimes_{\mathcal{O}_{K_{0}}} D_{\text {cris }}(V),
$$

then $\left(s_{\alpha}\right) \subset(\mathfrak{M} / u \mathfrak{M})^{\otimes} \subset D_{\text {cris }}(V)^{\otimes}$.

(2) If $k^{\text {sep }}$ denotes a separable closure of $k$, then there exists a $W\left(k^{\text {sep }}\right)$-linear isomorphism

$$
L \otimes_{\mathbb{Z}_{p}} W\left(k^{\mathrm{sep}}\right) \stackrel{\sim}{\longrightarrow} \mathfrak{M} / u \mathfrak{M} \otimes_{W(k)} W\left(k^{\mathrm{sep}}\right)
$$

taking $s_{\alpha}$ to $s_{\alpha}$. In particular, $\left(s_{\alpha}\right) \subset(\mathfrak{M} / u \mathfrak{M})^{\otimes}$ defines a reductive subgroup $G^{\prime}$ of $\mathrm{GL}(\mathfrak{M} / u \mathfrak{M})$, which is a pure inner form of $G$.

(3) If $k$ is finite and $G$ is connected, then there exists a $W$-linear isomorphism

$$
L \otimes_{\mathbb{Z}_{p}} W \stackrel{\sim}{\longrightarrow} \mathfrak{M} / u \mathfrak{M}
$$

taking $s_{\alpha}$ to $s_{\alpha}$. In particular $\left(s_{\alpha}\right) \subset(\mathfrak{M} / u \mathfrak{M})^{\otimes}$ defines a reductive subgroup $G^{\prime}$ of $\mathrm{GL}(\mathfrak{M} / u \mathfrak{M})$, which is isomorphic to $G \times \times_{\operatorname{Spec} \mathbb{Z}_{p}} \operatorname{Spec} W$.

Proof. (1) and (2) follow from (1.3.4); in fact (1) holds for any $G_{K}$-invariant tensors, without assuming that $G$ is reductive. To see that $G^{\prime}$ is a pure inner form of $G$ in (2), note that specializing the torsor $P$ which appears in the proof of (1.3.4) at $u=0$ gives a class in $H^{1}(\operatorname{Spec} W, G)$, and $G^{\prime}$ can be obtained from $G$ by twisting by this class.

Finally, (3) follows from (1.3.5) once we remark that $s_{\alpha} \in D_{\text {cris }}(V)^{\otimes}$ is equal to

$$
\left.\tilde{s}_{\alpha}\right|_{u=0}: \mathbf{1} \rightarrow(\mathfrak{M} / u \mathfrak{M})^{\otimes} \hookrightarrow D_{\text {cris }}(V)^{\otimes},
$$

the final inclusion being given by the first isomorphism of (1.2.1)(1). The equality is a formal consequence of the functoriality of this isomorphism.

(1.4) Application to $p$-divisible groups. We now apply the previous results to $p$-divisible groups over $\mathcal{O}_{K}$.

If $R$ is a ring which is $p$-adically separated and complete, and $\mathscr{G}$ is a $p$-divisible group over $R$, we will denote by $\mathbb{D}(\mathscr{G})$ the corresponding (contravariant) Frobenius crystal on the crystalline site of $R$.

If $R^{\prime} \rightarrow R$ is a surjection whose kernel is equipped with divided powers and is topologically nilpotent for the $p$-adic topology, then we denote by $\mathbb{D}(\mathscr{G})\left(R^{\prime}\right)$ the 
value of $\mathbb{D}(\mathscr{G})$ on $R^{\prime}$. In particular, there is a short exact sequence of finite, locally free $R$-modules

$$
0 \rightarrow(\operatorname{Lie} \mathscr{G})^{*} \rightarrow \mathbb{D}(\mathscr{G})(R) \rightarrow \operatorname{Lie} \mathscr{G}^{*} \rightarrow 0,
$$

where the term on the left is the $R$-dual of $\operatorname{Lie} \mathscr{G}$, and $\mathscr{G}^{*}$ is the Cartier dual of $\mathscr{G}$. If $R^{\prime} \rightarrow R$ is as above, we denote by $\mathrm{Fil}^{1} \mathbb{D}(\mathscr{G})\left(R^{\prime}\right)$ the preimage of $(\mathrm{Lie} \mathscr{G})^{*}$ in $\mathbb{D}(\mathscr{G})\left(R^{\prime}\right)$.

Let $S$ be the $p$-adic completion of $W\left[u, E(u)^{n} / n !\right]_{n \geq 1}$. We equip it with a Frobenius $\varphi$ which sends $u$ to $u^{p}$. The ideal $\operatorname{Fil}^{1} S=\operatorname{ker}\left(S \stackrel{u \mapsto}{\rightarrow}^{\pi} \mathcal{O}_{K}\right)$ is equipped with divided powers. In particular, if $\mathscr{G}$ is a $p$-divisible group over $\mathcal{O}_{K}$, then $\mathbb{D}(\mathscr{G})(S)$ is a finite free module, equipped with a semi-linear Frobenius $\varphi$ and a submodule $\mathrm{Fil}^{1} \mathbb{D}(\mathscr{G})(S)$. In fact $\varphi\left(\mathrm{Fil}^{1} \mathbb{D}(\mathscr{G})(S)\right) \subset p \mathbb{D}(\mathscr{G})(S)$, but we will not need this explicitly.

(1.4.1) Let $\mathrm{BT}_{/ \mathfrak{S}}^{\varphi}$ denote the full subcategory of $\operatorname{Mod}_{/ \mathfrak{S}}^{\varphi}$ consisting of objects $\mathfrak{M}$ such that $\mathfrak{M}$ is $\varphi$-stable and the cokernel of $\varphi^{*}(\mathfrak{M}) \rightarrow \mathfrak{M}$ is killed by $E(u)$.

We view $S$ as a $\mathfrak{S}$-algebra by sending $u$ to $u$. If $\mathfrak{M}$ is in $\mathrm{BT}_{/ \mathfrak{S}}^{\varphi}$, then following Breuil $\left[\mathrm{Br}\right.$, we set $\mathcal{M}(\mathfrak{M})=S \otimes_{\mathfrak{S}} \varphi^{*}(\mathfrak{M})$ and equip $\mathcal{M}(\mathfrak{M})$ with the induced Frobenius $\varphi$, and with an $S$-submodule

$$
\mathrm{Fil}^{1} \mathcal{M}(\mathfrak{M})=\left\{x \in S \otimes_{\varphi, \mathfrak{S}} \mathfrak{M}: 1 \otimes \varphi(x) \in \mathrm{Fil}^{1} S \otimes_{\mathfrak{S}} \mathfrak{M} \subset S \otimes_{\mathfrak{S}} \mathfrak{M}\right\} .
$$

One checks easily that

$$
\operatorname{Fil}^{1} \mathcal{M}(\mathfrak{M})=S \cdot \operatorname{Fil}^{1}\left(\varphi^{*}(\mathfrak{M})\right)+\operatorname{Fil}^{1} S \cdot \mathcal{M} .
$$

We denote by $\left(p\right.$-div $\left./ \mathcal{O}_{K}\right)$ the category of $p$-divisible groups over $\mathcal{O}_{K}$.

Theorem (1.4.2). The functor $\mathfrak{M}$ of (1.2.1) induces a fully faithful contravariant functor

$$
\mathfrak{M}:\left(p-\operatorname{div} / \mathcal{O}_{K}\right) \rightarrow \mathrm{BT}_{/ \mathfrak{S}}^{\varphi} ; \quad \mathscr{G} \mapsto \mathfrak{M}(\mathscr{G}):=\mathfrak{M}\left(T_{p} \mathscr{G}^{*}\right),
$$

which is an equivalence when $p>2$. If $\mathscr{G}$ is a $p$-divisible group over $\mathcal{O}_{K}$ and either $p>2$ or $\mathscr{G}^{*}$ is connected, then there is a canonical isomorphism

$$
\mathbb{D}(\mathscr{G})(S) \stackrel{\sim}{\longrightarrow} \mathcal{M}(\mathfrak{M}(\mathscr{G}))
$$

compatible with $\varphi$ and filtrations.

Proof. That $\mathfrak{M}$ induces a functor as claimed follows from [Ki 1, 2.2.7] and its proof. If $p>2, \mathfrak{M}$ is in $\mathrm{BT}_{/ \mathfrak{S}}^{\varphi}$ and $\mathscr{G}=\mathscr{G}(\mathfrak{M})$ is the corresponding $p$-divisible group, then the construction of the quasi-inverse to $\mathfrak{M}$ in loc. cit shows that $\mathbb{D}(\mathscr{G})(S) \stackrel{\sim}{\longrightarrow}$ $\mathcal{M}(\mathfrak{M}(\mathscr{G}))$. If $p=2$, then this formula holds if $\mathscr{G}^{*}$ is connected [Ki 3, 1.1.6(2), $1.2 .8][4$

Corollary (1.4.3). Let $\mathscr{G}$ be a $p$-divisible group over $\mathcal{O}_{K}$, and if $p=2$ assume that $\mathscr{G}^{*}$ is connected. Let $L=T_{p} \mathscr{G}^{*}, \mathfrak{M}=\mathfrak{M}(L)=\mathfrak{M}(\mathscr{G})$ and $\left(s_{\alpha}\right) \subset L^{\otimes}$ be a collection of $G_{K}$-invariant tensors defining a reductive subgroup $G \subset \operatorname{GL}(L)$. Then

(1) There is a canonical $\varphi$-equivariant isomorphism $\varphi^{*}(\mathfrak{M} / u \mathfrak{M}) \stackrel{\sim}{\longrightarrow} \mathbb{D}\left(\mathscr{G}_{0}\right)(W)$, where $\mathscr{G}_{0}=\mathscr{G} \otimes_{\mathcal{O}_{K}} k$.

\footnotetext{
${ }^{4}$ Note that the correspondence of $\mathrm{Ki} 3$ is covariant.
} 
(2) There exists a $W\left(k^{\mathrm{sep}}\right)$-linear isomorphism

$$
L \otimes_{\mathbb{Z}_{p}} W\left(k^{\mathrm{sep}}\right) \stackrel{\sim}{\longrightarrow} \mathbb{D}\left(\mathscr{G}_{0}\right)(W) \otimes_{W} W\left(k^{\mathrm{sep}}\right)
$$

taking $s_{\alpha}$ to $\varphi^{*}\left(s_{\alpha}\right) \in \mathbb{D}\left(\mathscr{G}_{0}\right)(W)^{\otimes}$. In particular, $\left(\varphi^{*}\left(s_{\alpha}\right)\right) \subset \mathbb{D}\left(\mathscr{G}_{0}\right)(W)^{\otimes}$ defines a reductive subgroup $G_{W} \subset \mathrm{GL}\left(\mathbb{D}\left(\mathscr{G}_{0}\right)(W)\right)$ which is an inner form of $G$.

(3) If $G$ is connected and $k$ is finite, then there exists a $W$-linear isomorphism

$$
L \otimes_{\mathbb{Z}_{p}} W \stackrel{\sim}{\longrightarrow} \mathbb{D}\left(\mathscr{G}_{0}\right)(W)
$$

taking $s_{\alpha}$ to $\varphi^{*}\left(s_{\alpha}\right) \in \mathbb{D}\left(\mathscr{G}_{0}\right)(W)^{\otimes}$. In particular, $\left(\varphi^{*}\left(s_{\alpha}\right)\right) \subset \mathbb{D}\left(\mathscr{G}_{0}\right)(W)^{\otimes}$ defines a reductive subgroup $G_{W} \subset \mathrm{GL}\left(\mathbb{D}\left(\mathscr{G}_{0}\right)(W)\right)$ which is isomorphic to $G \times \times_{\text {Spec }} \mathbb{Z}_{p} W$.

(4) The filtration $\mathrm{Fil}^{1} \mathbb{D}\left(\mathscr{G}_{0}\right)(k) \subset \mathbb{D}\left(\mathscr{G}_{0}\right)(k)$ is given by a cocharacter

$$
\mu_{0}: \mathbb{G}_{m} \rightarrow G_{W} \otimes_{W} k .
$$

Proof. For (1) note that if we view $W$ as an $S$-algebra by sending $u$ to 0 , then using (1.4.2) we have

$$
\begin{aligned}
& \mathbb{D}\left(\mathscr{G}_{0}\right)(W) \stackrel{\sim}{\longrightarrow} \mathbb{D}(\mathscr{G})(S) \otimes_{S} W \stackrel{\sim}{\longrightarrow} \mathcal{M}(\mathfrak{M}(\mathscr{G})) \otimes_{S} W \\
&=\varphi^{*}\left(\mathfrak{M}\left(T_{p} \mathscr{G}^{*}\right)\right) \otimes_{\mathfrak{S}} W=\varphi^{*}(\mathfrak{M}) / u \varphi^{*}(\mathfrak{M}) .
\end{aligned}
$$

In particular, we may view the tensors $\varphi^{*}\left(s_{\alpha}\right)$ in $\mathbb{D}\left(\mathscr{G}_{0}\right)(W)^{\otimes}$ by $(1.3 .6)(1)$, and (2) follows from (1.3.6)(2). Similarly, (3) follows from (1.3.6)(3).

By (1.3.4), $\left(s_{\alpha}\right) \subset L^{\otimes}$ gives rise to a collection of $\varphi$-invariant tensors $\left(\tilde{s}_{\alpha}\right) \subset \mathfrak{M}^{\otimes}$, which define a reductive subgroup $G_{\mathfrak{S}} \subset \operatorname{GL}(\mathfrak{M})$. Pulling these back by $\varphi$ and specializing at $u=\pi$ we obtain tensors

$$
s_{\alpha, K}=\left.\varphi^{*}\left(\tilde{s}_{\alpha}\right)\right|_{u=\pi} \in\left(\varphi^{*}(\mathfrak{M}) \otimes_{\mathfrak{S}} \mathcal{O}_{K}\right)^{\otimes},
$$

which define a reductive subgroup $G_{\mathcal{O}_{K}} \subset \mathrm{GL}\left(\varphi^{*}(\mathfrak{M}) \otimes_{\mathfrak{S}} \mathcal{O}_{K}\right)$.

By (1.4.2), we have that $\varphi^{*}(\mathfrak{M}) \otimes_{\mathfrak{S}} \mathcal{O}_{K} \stackrel{\sim}{\longrightarrow} \mathbb{D}(\mathscr{G})\left(\mathcal{O}_{K}\right)$ is compatible with $\varphi$ and filtrations. Hence it suffices to show that the filtration on $\mathbb{D}(\mathscr{G})\left(\mathcal{O}_{K}\right)$ is given by a cocharacter $\mathbb{G}_{m} \rightarrow G_{\mathcal{O}_{K}}$, that is, in the terminology of $\S 1$, that it is $G_{\mathcal{O}_{K}}$-split. By (1.1.4) it suffices to show that the induced filtration on $\mathbb{D}(\mathscr{G})\left(\mathcal{O}_{K}\right) \otimes_{\mathcal{O}_{K}} K$ is $\left.G_{\mathcal{O}_{K}}\right|_{K}$-split. Now using (1.2.1) and (1.4.2) we have

$$
D_{\mathrm{dR}}(V) \stackrel{\sim}{\longrightarrow} \varphi^{*}(\mathfrak{M}) \otimes_{\mathfrak{S}} K \stackrel{\sim}{\longrightarrow} \mathbb{D}(\mathscr{G})\left(\mathcal{O}_{K}\right) \otimes_{\mathcal{O}_{K}} K,
$$

where $V=L[1 / p]$. The compatibility with filtrations in (1.2.1)(1) and (1.4.2) shows that this is an isomorphism of filtered $K$-vector spaces, so we have to show that the filtration on $D_{\mathrm{dR}}(V)$ is $\left.G_{\mathcal{O}_{K}}\right|_{K}$-split. This follows from (1.4.5) below.

Lemma (1.4.5). Let $D$ be a weakly admissible filtered $K_{0}$-module, and let $\left(s_{\alpha}\right) \subset$ $\mathrm{Fil}^{0} D^{\otimes}$ be a collection of $\varphi$-invariant tensors defining a reductive subgroup $G \subset$ $\mathrm{GL}(D)$. Then the filtration on $D_{K}=D \otimes_{K_{0}} K$ is $G \otimes_{K_{0}} K$-split.

Proof. Let $\langle D\rangle^{\otimes}$ be the Tannakian category generated by the weakly admissible module $D$. Let $\omega$ be the fibre functor on $\langle D\rangle^{\otimes}$ given by $\omega(W)=W \otimes_{K_{0}} K$. Since the $s_{\alpha} \in \mathrm{Fil}^{0} D^{\otimes}$ we have

$$
\operatorname{Aut}^{\otimes}(\omega) \subset G \otimes_{K_{0}} K \subset \mathrm{GL}\left(D \otimes_{K_{0}} K\right) .
$$

By (1.1.3) the filtration on $D \otimes_{K_{0}} K$ is $\operatorname{Aut}^{\otimes}(\omega)$-split and hence $G \otimes_{K_{0}} K$-split. 
(1.4.6) We remark that the condition in the corollary when $p=2$ is one of the two points which force us to assume that $p>2$.

The statement of (1.3.6)(2) was conjectured by Milne [Mi 3], without the restriction at $p=2$, which should be unnecessary. See also Va 3 .

(1.5) Deformation theory. We keep the notation of the previous section.

Let $\mathscr{G}_{0}$ be a $p$-divisible group over $k$. We recall the explicit description of the versal deformation ring of $\mathscr{G}_{0}$ given by Faltings [Fa, $\left.\S 7\right]$ (see also [Mo, $\left.\S 4\right]$ ).

Fix a cocharacter $\mu: \mathbb{G}_{m} \rightarrow \mathrm{GL}\left(\mathbb{D}\left(\mathscr{G}_{0}\right)(W)\right)$ whose reduction $\bmod p, \mu_{0}$, gives rise to the filtration on $\mathbb{D}\left(\mathscr{G}_{0}\right)(k)$. The filtration on $\mathbb{D}(\mathscr{G})(W)$ defined by $\mu$ corresponds to a $p$-divisible group $\mathscr{G}$ over $W$ lifting $\mathscr{G}_{0} \mathrm{Me}$.

Let $U^{\circ} \subset \operatorname{GL}\left(\mathbb{D}\left(\mathscr{G}_{0}\right)(W)\right)$ be the opposite unipotent defined by $\mu$ and let $R$ be the complete local ring at the identity section of $U^{\circ}$. Then $R \stackrel{\sim}{\longrightarrow} W \llbracket t_{1}, \ldots, t_{n} \rrbracket$ is a power series ring over $W$, where

$$
n=\operatorname{dim} U^{\circ}=\operatorname{dim}_{k} \operatorname{gr}^{-1} \operatorname{End}_{k} \mathbb{D}\left(\mathscr{G}_{0}\right)(k) .
$$

We equip $R$ with a Frobenius $\varphi=\varphi_{R}$ sending $t_{i}$ to $t_{i}^{p}$ and acting as the usual Frobenius on $W$.

Write $M_{0}=\mathbb{D}\left(\mathscr{G}_{0}\right)(W)$ equipped with the filtration $\mathrm{Fil}^{1} M_{0}$, induced by the chosen character $\mu$ and Frobenius $\varphi=\varphi_{M_{0}}$. Let $M=M_{0} \otimes_{W} R$ with the filtration induced from $M_{0}$. We equip $M$ with a semi-linear Frobenius $\varphi$ given by the composite

$$
M=M_{0} \otimes_{W} R \stackrel{\varphi \otimes \varphi}{\rightarrow} M \stackrel{u}{\rightarrow} M,
$$

where $u \in U^{\circ}(R)$ is the tautological $R$-point of $U^{\circ}$.

Then there is a $p$-divisible group $\mathscr{G}_{R}$ over $R$ such that $\mathscr{G}_{R} \otimes_{R} R /\left(t_{1}, \ldots, t_{n}\right) \stackrel{\sim}{\longrightarrow}$ $\mathscr{G}$ and $\mathscr{G}_{R}$ is a versal deformation of $\mathscr{G}_{0}$, and an isomorphism $\mathbb{D}\left(\mathscr{G}_{R}\right)(R) \stackrel{\sim}{\longrightarrow} M$ compatible with Frobenius and filtration [Mo 4.5]. Since $R$ is formally smooth, the $F$-crystal structure on $\mathbb{D}\left(\mathscr{G}_{R}\right)$ is given by a connection $\nabla: M \rightarrow M \otimes \Omega_{R}^{1}$, such that $\varphi^{*}(M) \rightarrow M$ is parallel. That is, it is compatible with the connections $\varphi^{*}(\nabla)$ and $\nabla$ on $\varphi^{*}(M)$ and $M$, respectively.

If $R^{\prime}$ is any $p$-adically complete, $p$-torsion free $W$-algebra, equipped with a lift of Frobenius $\varphi_{R^{\prime}}$ and $\iota: R \rightarrow R^{\prime}$ is a map of $W$-algebras, then the Frobenius on

$$
M_{R^{\prime}}:=M \otimes_{R} R^{\prime} \stackrel{\sim}{\longrightarrow} \mathbb{D}\left(\mathscr{G}_{R} \otimes_{R} R^{\prime}\right)\left(R^{\prime}\right)
$$

is given as follows: Since $\varphi_{R^{\prime}} \circ \iota$ and $\iota \circ \varphi_{R}$ have the same reduction modulo $p$ (we do not assume they are equal), the structure of a crystal on $\mathbb{D}\left(\mathscr{G}_{R}\right)$ induces a canonical isomorphism $\varepsilon: \varphi_{R^{\prime}}^{*}{ }^{*} M \stackrel{\sim}{\longrightarrow} \iota^{*} \varphi_{R}^{*} M$, and the Frobenius $\varphi_{R^{\prime}}$ on $M_{R^{\prime}}$ is given by

$$
\varphi_{R^{\prime}}^{*}\left(M_{R^{\prime}}\right)=\varphi_{R^{\prime}}^{*} \iota^{*} M \underset{\varepsilon}{\stackrel{\sim}{\longrightarrow}} \iota^{*} \varphi_{R}^{*} M \rightarrow \iota^{*} M=M_{R^{\prime}} .
$$

The map $\varepsilon$ is compatible with the connections $\varphi_{R^{\prime}}^{*}{ }^{*}(\nabla)$ and $\iota^{*} \varphi_{R}^{*}(\nabla)$ on $\varphi_{R^{\prime}}^{*} \iota^{*} M$ and $\iota^{*} \varphi_{R}^{*} M$, respectively. This compatibility follows from the cocycle condition in the definition of a crystal, or equivalently the integrability of $\nabla$. In particular, one sees that (1.5.1) is parallel for $\nabla_{R^{\prime}}=\iota^{*}(\nabla)$.

Explicitly, $\varepsilon$ has the following description [Fa, p. 134], [Mo, 4.3]: If $\underline{i}=\left(i_{1}, \ldots, i_{n}\right)$ is a multi-index, write $\nabla(\partial)^{\underline{i}}=\nabla\left(\partial t_{1}\right)^{i_{1}} \ldots \nabla\left(\partial t_{n}\right)^{i_{n}}$ and $z^{\underline{i}}=z_{1}^{i_{1}} \ldots z_{n}^{i_{n}}$, where 


$$
\begin{aligned}
& z_{i}=\varphi_{R^{\prime}} \circ \iota\left(t_{i}\right)-\iota \circ \varphi_{R}\left(t_{i}\right) \text {. Then } \\
& \qquad \varepsilon(m \otimes 1)=\sum_{\underline{i}} \nabla(\partial)^{\underline{i}}(m \otimes 1) \otimes \frac{z^{\underline{i}}}{\underline{i} !} .
\end{aligned}
$$

We will need the following lemma relating the action of $\varphi$ and $\nabla_{R^{\prime}} 5$

Lemma (1.5.2). Let $\iota: R \rightarrow R^{\prime}$ be as above. Suppose that $\iota \circ \varphi_{R}=\varphi_{R^{\prime}} \circ \iota$ and that there is a smooth subgroup $H \subset \mathrm{GL}\left(M_{0}\right)$, such that the composite

$$
\theta: M_{0} \stackrel{m \mapsto \varphi^{-1}(m) \otimes 1}{\longrightarrow} \varphi^{*}\left(M_{R^{\prime}}\right) \rightarrow M_{R^{\prime}}=M_{0} \otimes_{W} R^{\prime}
$$

is given by $\theta(m)=A \cdot m$ for some $A \in H\left(R^{\prime}\right)$. Then $\beta: M_{0} \stackrel{\nabla_{R^{\prime}}}{\rightarrow} M_{R^{\prime}} \otimes_{R^{\prime}} \Omega_{R^{\prime}}^{1}$, the linear map induced by $\nabla_{R^{\prime}}$, is given by an element of Lie $H \otimes_{W} \Omega_{R^{\prime}}^{1}$.

Proof. Note that $\theta$ is well defined, without inverting $p$, as $\iota \circ \varphi_{R}=\varphi_{R^{\prime}} \circ \iota$. It suffices to prove the lemma with $\iota(R)$ in place of $R^{\prime}$, so we may assume that $\iota$ is surjective.

Since the map $\varphi^{*}\left(M_{R^{\prime}}\right) \rightarrow M_{R^{\prime}}$ is parallel for $\nabla_{R^{\prime}}$ one computes that $d A+\beta A=$ $A \varphi(\beta)$ as elements of End $M_{R^{\prime}} \otimes_{R^{\prime}} \Omega_{R^{\prime}}^{1}[1 / p]$, where $\varphi(\beta)$ is the composite

$$
\begin{aligned}
M_{0} \stackrel{\varphi^{-1}}{\rightarrow} M_{0}[1 / p] & \stackrel{\nabla_{R^{\prime}}}{\rightarrow} M_{R^{\prime}} \otimes_{R^{\prime}} \Omega_{R^{\prime}}^{1}[1 / p] \\
& =M_{0} \otimes_{W} \Omega_{R^{\prime}}[1 / p] \stackrel{\varphi \otimes \varphi}{\rightarrow} M_{0} \otimes_{W} \Omega_{R^{\prime}}[1 / p]=M_{R^{\prime}} \otimes_{R^{\prime}} \Omega_{R^{\prime}}^{1}[1 / p] .
\end{aligned}
$$

Hence $\varphi(\beta)=A^{-1} d A+A^{-1} \beta A \in$ End $_{R^{\prime}} M_{R^{\prime}} \otimes_{R^{\prime}} \Omega_{R^{\prime}}^{1}$ and

$$
\beta=-d A A^{-1}+A \varphi(\beta) A^{-1} \text {. }
$$

Repeatedly substituting the right-hand side of the above formula for $\beta$ one obtains a series whose $m^{\text {th }}$ term is contained in Lie $H \otimes_{W} R^{\prime} \cdot \varphi^{m-1}\left(\Omega_{R^{\prime}}^{1}\right)$. Since $\iota$ is $\varphi$-compatible and for any $\omega \in \Omega_{R}^{1}, \varphi^{m}(\omega) \in\left(t_{1}^{p^{m}-1}, \ldots, t_{r}^{p^{m}-1}\right) \Omega_{R}^{1}$, the series converges to an element of Lie $H \otimes_{W} \Omega_{R^{\prime}}^{1}$.

Corollary (1.5.3). We have:

(1) The map $\nabla: M_{0} \rightarrow M \otimes_{R} \Omega_{R}^{1}$ is given by an element of Lie $U^{\circ} \otimes_{W} \Omega_{R}^{1}$.

(2) For any $\iota: R \rightarrow R^{\prime}$ as in (1.5), the map

$$
\theta: M_{0} \rightarrow M_{R^{\prime}}[1 / p] ; \quad m \mapsto \varphi_{R^{\prime}}\left(\varphi^{-1}(m) \otimes 1\right)
$$

is given by an element of $U^{\circ}\left(R^{\prime}[1 / p]\right)$.

Proof. (1) follows from (1.5.2), applied with $\iota$ the identity map.

For (2), denote by $\varphi_{W}: R \rightarrow R$ the endomorphism given by $\varphi$ on $W$, and $\varphi_{W}\left(t_{i}\right)=t_{i}$ and by $\varphi_{R / W}$ the $W$-linear endomorphism of $R$ given by $\varphi_{R / W}\left(t_{i}\right)=t_{i}^{p}$. Then $\varphi_{R}=\varphi_{R / W} \circ \varphi_{W}$. We will also denote by $\varphi_{W}$ the $\varphi_{W}$-semi-linear map on $M_{R}=M_{0} \otimes_{W} R$, given by $\left.\varphi\right|_{M_{0}} \otimes \varphi_{W}$.

To show (2) we first note that the composite

$$
\varphi_{W} \nabla \varphi_{W}^{-1}: M_{R} \stackrel{\varphi_{W}^{-1}}{\rightarrow} M_{R}[1 / p] \stackrel{\nabla^{\prime}}{\rightarrow} M_{R} \otimes_{R} \Omega_{R}^{1}[1 / p] \stackrel{\varphi_{W} \otimes_{\varphi_{W}}}{\rightarrow} M_{R} \otimes_{R} \Omega_{R}^{1}[1 / p]
$$

is given by an element of Lie $U^{\circ} \otimes_{W} \Omega_{R}^{1}[1 / p]$. Indeed, in the notation of the proof of (1.5.2), composing this map with $1 \otimes \varphi_{R / W}: M_{0} \otimes_{W} \Omega_{R}^{1}[1 / p] \rightarrow M_{0} \otimes_{W} \Omega_{R}^{1}[1 / p]$ produces the map

$$
\varphi(\beta)=A^{-1} d A+A^{-1} \beta A \in \operatorname{Lie} U^{\circ} \otimes_{W} \Omega_{R}^{1} .
$$

\footnotetext{
${ }^{5} \mathrm{Cf}$. the second remark in [Fa, p. 136].
} 
Hence, we compute for any $m \in M_{0}$,

$$
\begin{aligned}
\varphi_{R^{\prime}}\left(\varphi^{-1}(m) \otimes 1\right) & =\left(\iota^{*}\left(\varphi_{R} \otimes 1\right) \circ \varepsilon\right)\left(\varphi^{-1}(m) \otimes 1\right) \\
& =\iota^{*}\left(\varphi_{R} \otimes 1\right) \exp \left(\sum_{i} \nabla\left(\partial t_{i}\right) \otimes z_{i}\right)\left(\varphi^{-1}(m) \otimes 1\right) \\
& =\iota^{*}\left(\varphi_{R} \circ \varphi_{W}^{-1}\right) \exp \left(\sum_{i} \varphi_{W} \nabla\left(\partial t_{i}\right) \varphi_{W}^{-1} \otimes \varphi_{W}\left(z_{i}\right)\right)(m \otimes 1) .
\end{aligned}
$$

Since $\sum_{i} \varphi_{W} \nabla\left(\partial t_{i}\right) \varphi_{W}^{-1} \otimes \varphi_{W}\left(z_{i}\right)$ is in Lie $U^{\circ} \otimes_{W} R^{\prime}[1 / p]$, by what we saw above, and $\iota^{*}\left(\varphi_{R} \circ \varphi_{W}^{-1}\right)$ is given by the universal element $u \in U^{\circ}(R)$, this proves the lemma.

(1.5.4) Suppose $G \subset \operatorname{GL}\left(M_{0}\right)$ is a connected reductive subgroup defined by a family of $\varphi$-invariant tensors $\left(s_{\alpha}\right) \subset M_{0}^{\otimes}$, such that the filtration on $\mathbb{D}\left(\mathscr{G}_{0}\right)(k)$ is $G \otimes_{W} k$-split 6 Choose a cocharacter $\mu_{0}: \mathbb{G}_{m} \rightarrow G \otimes_{W} k$ inducing this filtration. We may take $\mu$ to be a character $\mu: \mathbb{G}_{m} \rightarrow G$ lifting $\mu_{0}$. Such a lifting always exists DG, XI, 4.2]. Let $\mathfrak{g}$ denote the Lie algebra of $G$. Then $\mu$ induces a grading of $\mathfrak{g}$.

Let $U_{G}^{\circ} \subset G$ be the opposite unipotent defined by $\mu$ and let $R_{G}$ be the complete local ring at the identity section of $U_{G}^{\circ}$. Then $R_{G}$ is a formally smooth quotient of $R$. We choose the coordinates $t_{i}$ so that $R_{G}=R /\left(t_{r+1}, \ldots, t_{n}\right)$, where $r=\mathrm{rk}_{W} \mathfrak{g} / \mathrm{Fil}^{0} \mathfrak{g}$.

Let $M_{R_{G}}=M \otimes_{R} R_{G}$ and let $\nabla_{R_{G}}$ be the induced connection on $M_{R_{G}}$. By (1.5.2), $\nabla_{R_{G}}$ induces a map $M_{0} \rightarrow M_{R_{G}} \otimes_{R_{G}} \Omega_{R_{G}}^{1}$ which is in Lie $U_{G}^{\circ} \otimes_{W} \Omega_{R_{G}}^{1}$. In particular we have $\nabla_{R_{G}}\left(s_{\alpha} \otimes 1\right)=0$. Hence $s_{\alpha} \otimes 1$ may be viewed as a morphism of crystals $\mathbf{1} \rightarrow \mathbb{D}\left(\mathscr{G}_{R} \otimes_{R} R_{G}\right)^{\otimes}$. In particular, for any map $\iota: R \rightarrow R^{\prime}$ as in (1.5), if $\iota$ factors through $R_{G}$, then $\varepsilon\left(s_{\alpha} \otimes 1\right)=s_{\alpha} \otimes 1$. (This may also be seen directly from the explicit description of $\varepsilon$ in terms of $\nabla$.)

(1.5.5) We want to describe which points of $R$ come from $R_{G}$-points. In $\mathrm{Mo}$, $4.9]$ this is done for points with values in a power series ring over $W$, following a remark of Faltings [Fa, p. 136]. We will need to consider the case of the ring of integers in a finite extension of $K_{0}$. For this we need some preparation.

Consider the ring $K_{0} \llbracket u \rrbracket$ equipped with the Frobenius given by the usual Frobenius on $K_{0}$ and sending $u$ to $u^{p}$. Let $D_{0}$ be a finite dimensional $K_{0}$-vector space, and set $D=D_{0} \otimes_{K_{0}} K_{0} \llbracket u \rrbracket$. Given an isomorphism $\varphi^{*}(D) \stackrel{\sim}{\longrightarrow} D$, there is a unique $\varphi$-equivariant section $\xi: D_{0}=D / u D \rightarrow D$. To construct it choose any $K_{0}$-linear section $\xi_{0}: D / u D \rightarrow D$, and set (cf. [Ki 1, 1.2.6])

$$
\xi=\lim _{n} \varphi^{n} \circ \xi_{0} \circ \varphi^{-n} .
$$

The connection $\nabla_{D}$ on $D$, such that $\nabla_{D}\left(\xi\left(D_{0}\right)\right)=0$ is the unique connection for which $\varphi^{*}(D) \stackrel{\sim}{\longrightarrow} D$ is parallel.

Suppose that $D_{0}$ is equipped with a decreasing filtration $D_{0}^{\bullet}$, and equip $D$ with the induced filtration $D^{\bullet}$.

Lemma (1.5.6). Suppose that $D_{0}$ with its Frobenius and filtration is a weakly admissible $K_{0}$-module. Let $\left(s_{\alpha}\right) \subset \operatorname{Fil}^{0}\left(D_{0}^{\otimes}\right)$ be a collection of $\varphi$-invariant tensors defining a reductive subgroup $G \subset \mathrm{GL}\left(D_{0}\right)$, and fix a cocharacter $\mu: \mathbb{G}_{m} \rightarrow G$ inducing the filtration $D_{0}^{\bullet}$. Suppose that:

\footnotetext{
${ }^{6}$ Note that, in general, there is a well defined Frobenius only on $M_{0}[1 / p]^{\otimes}$ and not $M_{0}^{\otimes}$, as the dual module $M_{0}^{*}$ need not be Frobenius stable. A tensor in $M_{0}^{\otimes}$ is said to be $\varphi$-invariant if it is $\varphi$-invariant in $M_{0}[1 / p]^{\otimes}$.
} 
(1) For each $\alpha, \xi\left(s_{\alpha}\right) \in \mathrm{Fil}^{0} D^{\otimes}$.

(2) If $U^{\circ} \subset \mathrm{GL}\left(D_{0}\right)$ denotes the opposite unipotent defined by $\mu$, then the $K_{0} \llbracket u \rrbracket$-linear automorphism of $D$,

$$
\theta: D=D_{0} \otimes_{K_{0}} K_{0} \llbracket u \rrbracket \rightarrow D: \quad m_{0} \otimes a \mapsto \varphi\left(\varphi^{-1}\left(m_{0}\right) \otimes 1\right) \otimes a
$$

lies in $U^{\circ}\left(K_{0} \llbracket u \rrbracket\right)$.

Then $\xi\left(s_{\alpha}\right)=s_{\alpha} \otimes 1 \in D^{\otimes}$ for each $\alpha$, and the automorphism $\theta$ lies in the opposite unipotent subgroup $U_{G}^{\circ} \subset G$ defined by $\mu$.

Proof. We remark that a cocharacter $\mu$ as in the lemma always exists by (1.4.5). Since $G$ is defined by a finite subset of the $s_{\alpha}$, we may assume that the collection of tensors $\left(s_{\alpha}\right)$ is indexed by some finite set $I$.

Each $s_{\alpha}$ generates a $\varphi$-invariant line in a weakly admissible submodule of $D_{0}^{\otimes}$, so this line cannot lie in filtration degree 1 , and $s_{\alpha}$ gives rise to a morphism $\mathbf{1} \rightarrow D_{0}^{\otimes}$ in the category of (Ind-)weakly admissible modules. Now consider the map

$$
\text { End }_{K_{0}} D_{0} \rightarrow\left(D_{0}^{\otimes}\right)^{|I|} ; \quad g \mapsto\left(g\left(s_{\alpha}\right)\right)_{\alpha \in I} .
$$

This is a map of (Ind)-weakly admissible filtered $\varphi$-modules, and hence is strict for filtrations. In particular, if $g \in \operatorname{End}_{K_{0}} D_{0}$ maps $\left(s_{\alpha}\right)_{\alpha \in I}$ into $\operatorname{Fil}^{0}\left(D_{0}^{\otimes}\right)^{|I|}$, then $g \in \operatorname{Lie} P+\operatorname{Lie} G$, where $P \subset \operatorname{GL}\left(D_{0}\right)$ is the parabolic corresponding to $D_{0}^{\bullet}$.

Let $D_{n}=D / u^{n+1} D$ and let $\theta_{n}$ be the automorphism of $D_{n}$ induced by $\theta$. We will show by induction on $n$ that $\xi\left(s_{\alpha}\right)=s_{\alpha} \otimes 1$ modulo $u^{n}$. Note that this implies that

$$
\theta_{n-1}\left(s_{\alpha} \otimes 1\right)=\varphi\left(s_{\alpha} \otimes 1\right)=\varphi\left(\xi\left(s_{\alpha}\right)\right)=\xi\left(s_{\alpha}\right)=s_{\alpha} \otimes 1
$$

so that $\theta_{n-1} \in U_{G}^{\circ}\left(K_{0} \llbracket u \rrbracket / u^{n}\right)$.

Suppose $\xi\left(s_{\alpha}\right)=s_{\alpha} \otimes 1$ modulo $u^{n}$, and let $\tilde{\theta}_{n-1} \in U_{G}^{\circ}\left(K_{0} \llbracket u \rrbracket / u^{n+1}\right)$ be any lift of $\theta_{n-1}$. Then

$$
\theta_{n} \circ \tilde{\theta}_{n-1}^{-1}\left(s_{\alpha} \otimes 1\right)=\varphi\left(s_{\alpha} \otimes 1\right)=\xi\left(s_{\alpha}\right)=w\left(s_{\alpha} \otimes 1\right)
$$

for some $w \in \operatorname{ker}\left(U^{\circ}\left(K_{0} \llbracket u \rrbracket / u^{n+1}\right) \rightarrow U^{\circ}\left(K_{0} \llbracket u \rrbracket / u^{n}\right)\right)$. Writing $w=1+u^{n} w_{0}$ with $w_{0} \in \operatorname{Lie} U^{\circ}$, we have

$$
u^{n} w_{0}\left(s_{\alpha} \otimes 1\right)=\xi\left(s_{\alpha}\right)-s_{\alpha} \otimes 1 \in \operatorname{Fil}^{0}\left(D_{n}^{\otimes}\right),
$$

so $w_{0} s_{\alpha} \in \mathrm{Fil}^{0} D_{0}^{\otimes}$. It follows that

$$
w_{0} \in \operatorname{Lie} U^{\circ} \cap(\operatorname{Lie} P+\operatorname{Lie} G)=\operatorname{Lie} U^{\circ} \cap\left(\operatorname{Lie} P+\operatorname{Lie} U_{G}^{\circ}\right)=\operatorname{Lie} U_{G}^{\circ} .
$$

Hence $w \in U_{G}^{\circ}\left(K_{0} \llbracket u \rrbracket / u^{n+1}\right)$, so $\xi\left(s_{\alpha}\right)=s_{\alpha} \otimes 1$, and $\theta \in U_{G}^{\circ}\left(K_{0} \llbracket u \rrbracket\right)$.

(1.5.7) We now return to the notation and assumptions of (1.5.4). We will need a variant of the ring $S$. Let $I^{[n]} \subset S$ denote the divided powers of $I=\operatorname{ker}(S \rightarrow$ $\mathcal{O}_{K}$ ), and set7 $\widehat{S}=\lim _{n} S / I^{[n]}$. If $p>2$, the divided powers of $p$ are topologically nilpotent, and this is the same as the completion of $S$ with respect to the topology defined by the divided powers of the closure of the ideal $\left(u^{e i} / i !\right)_{i \geq 1}$. In particular, when $p>2$, we may regard $\widehat{S}$ as a subring of $K_{0} \llbracket u \rrbracket$, containing $S$.

Proposition (1.5.8). Suppose $p>2$ or that $\mathscr{G}_{0}^{*}$ is connected. Let $\varpi: R \rightarrow \mathcal{O}_{K}$ be a map of $W$-algebras and denote by $\mathscr{G}_{\varpi}$ the induced $p$-divisible group over $\mathcal{O}_{K}$. Then $\varpi$ factors through $R_{G}$ if and only if there exists a collection of $\varphi$-invariant tensors $\left(\tilde{s}_{\alpha}\right) \subset \mathbb{D}\left(\mathscr{G}_{\varpi}\right)(S)^{\otimes}$ lifting $\left(s_{\alpha}\right) \subset \mathbb{D}\left(\mathscr{G}_{0}\right)(W)^{\otimes}$, such that:

\footnotetext{
${ }^{7}$ This is the ring considered in $\mathrm{Fa}$.
} 
(1) If $s_{\alpha, \mathcal{O}_{K}}$ denotes the image of $\tilde{s}_{\alpha}$ in $\mathbb{D}\left(\mathscr{G}_{\varpi}\right)\left(\mathcal{O}_{K}\right)^{\otimes}$, then

$$
\left(s_{\alpha, \mathcal{O}_{K}}\right) \subset \operatorname{Fil}^{0}\left(\mathbb{D}\left(\mathscr{G}_{\varpi}\right)\left(\mathcal{O}_{K}\right)^{\otimes}\right) .
$$

(2) The $\left(\tilde{s}_{\alpha}\right)$ define a reductive subgroup $G_{S} \subset \operatorname{GL}\left(\mathbb{D}\left(\mathscr{G}_{\varpi}\right)(S)\right)$.

Proof. If $\varpi$ factors through $R_{G}$, we may lift it to a map $\tilde{\varpi}: R_{G} \rightarrow S$ and set $\tilde{s}_{\alpha}=\tilde{\varpi}\left(s_{\alpha} \otimes 1\right)$. Then the $\tilde{s}_{\alpha}$ clearly satisfy (1) and (2). We have to check that the $\tilde{\varpi}\left(s_{\alpha} \otimes 1\right)$ are $\varphi$-invariant. The Frobenius on $M_{S}:=\mathbb{D}\left(\mathscr{G}_{\varpi}\right)(S)=M_{R_{G}} \otimes_{R_{G}} S$ is given by

$$
\varphi^{*}\left(M_{S}\right)=\varphi^{*} \tilde{\varpi}^{*}\left(M_{R_{G}}\right) \underset{\varepsilon}{\stackrel{\sim}{\longrightarrow}} \tilde{\varpi}^{*} \varphi^{*}\left(M_{R_{G}}\right) \stackrel{\tilde{\varpi}^{*}(\varphi \otimes 1)}{\longrightarrow} \tilde{\varpi}^{*}\left(M_{R_{G}}\right),
$$

where $\varepsilon$ is as in (1.5). Now we have

$$
\tilde{\varpi}^{*}(\varphi \otimes 1) \circ \varepsilon\left(\tilde{\varpi}\left(s_{\alpha} \otimes 1\right)\right)=\tilde{\varpi}^{*}(\varphi \otimes 1)\left(\tilde{\varpi}\left(s_{\alpha} \otimes 1\right)\right)=\tilde{\varpi}\left(s_{\alpha} \otimes 1\right),
$$

where the first equality was seen in (1.5.4) and the second follows from the $\varphi$ invariance of $s_{\alpha}$.

Now suppose that there exist $\tilde{s}_{\alpha}$ satisfying (1) and (2). Denote by $\varpi_{0}: R \rightarrow W$ the map given by $\varpi_{0}\left(t_{i}\right)=0$ for $i=1, \ldots, n$. We denote by $\mathscr{G}_{\varpi \times \varpi_{0}}$ the $p$-divisible group over $\mathcal{O}_{K} \times_{k} W$ induced by $\varpi \times \varpi_{0}: R \rightarrow \mathcal{O}_{K} \times_{k} W$.

Assume first that $p>2$. Then the surjective map $W[u] \rightarrow \mathcal{O}_{K} \times_{k} W$ sending $u$ to $(\pi, 0)$ induces a map $\widehat{S} \rightarrow \mathcal{O}_{K} \times_{k} W$. Let $G_{\widehat{S}}=G_{S} \otimes_{S} \widehat{S}$. By (1.1.5) there is a $G_{\widehat{S}}$-split filtration on $\mathbb{D}\left(\mathscr{G}_{\varpi}(\widehat{S})\right)$ which simultaneously lifts the filtration on $\mathbb{D}\left(\mathscr{G}_{\varpi}\left(\mathcal{O}_{K}\right)\right)$ and the chosen filtration on $\mathbb{D}(\mathscr{G})(W)$. Since the kernel of $\widehat{S} \rightarrow \mathcal{O}_{K} \times_{k} W$ is equipped with topologically nilpotent divided powers, such a filtration corresponds to a $p$ divisible groun $8 \mathscr{G}_{\tilde{\varpi}}$ over $\widehat{S}$, deforming $\mathscr{G}_{\varpi \times \varpi_{0}}$. Since $R$ is a versal deformation ring for $\mathscr{G}_{0}, \mathscr{G}_{\tilde{\varpi}}$ is induced by a map $\tilde{\varpi}: R \rightarrow \widehat{S}$ lifting $\varpi \times \varpi_{0}$.

We may identify

$$
\mathbb{D}\left(\mathscr{G}_{\tilde{\varpi}}\right)(\widehat{S})=\mathbb{D}\left(\mathscr{G}_{\varpi}\right)(\widehat{S})=\mathbb{D}\left(\mathscr{G}_{\varpi}(S)\right) \otimes_{S} \widehat{S}
$$

with $M_{\widehat{S}}:=M_{R} \otimes_{R} \widehat{S}=M_{0} \otimes_{W} \widehat{S}$, and we view $\tilde{s}_{\alpha}$ as elements of $M_{\widehat{S}}^{\otimes}$. Consider the composite

$$
\varphi^{*}\left(M_{\widehat{S}}\right) \underset{\varepsilon}{\stackrel{\sim}{\longrightarrow}} \tilde{\varpi}^{*} \varphi^{*}\left(M_{R}\right) \stackrel{\tilde{\varpi}^{*}(\varphi \otimes 1)}{\longrightarrow} \tilde{\varpi}^{*}\left(M_{R}\right)=M_{\widehat{S}} .
$$

Since the filtration on $\mathbb{D}\left(\mathscr{G}_{\tilde{\varpi}}\right)(\widehat{S})$ is $G_{\widehat{S}^{-}}$split we have $\tilde{s}_{\alpha} \in \mathrm{Fil}^{0} \mathbb{D}\left(\mathscr{G}_{\varpi}\right)(\widehat{S})^{\otimes}$, and (1.5.3)(2) shows that the map $\theta: M_{0} \rightarrow M_{\widehat{S}}=M_{0} \otimes_{W} \widehat{S}$ is induced by an element of $U^{\circ}(\widehat{S}[1 / p])$. Hence, viewing $\tilde{s}_{\alpha}$ and $s_{\alpha} \otimes 1$ in $\left(M_{\widehat{S}} \otimes_{\widehat{S}} K_{0} \llbracket u \rrbracket\right)^{\otimes}$, and applying (1.5.6), we find that $\tilde{s}_{\alpha}=s_{\alpha} \otimes 1$ and that $\theta$ is induced by a point of $U_{G}^{\circ}\left(K_{0} \llbracket u \rrbracket\right) \cap$ $U^{\circ}(\widehat{S}[1 / p])=U_{G}^{\circ}(\widehat{S}[1 / p])$. In particular, each of the two maps in (1.5.10) sends $s_{\alpha} \otimes 1$ to $s_{\alpha} \otimes 1$. For $\varepsilon$ this holds as $\nabla_{\widehat{S}}\left(s_{\alpha} \otimes 1\right)=\nabla_{\widehat{S}}\left(\tilde{s}_{\alpha}\right)=0$ by (1.5.2), while the composite of the maps in (1.5.10) has this property since $\tilde{s}_{\alpha}$ is $\varphi$-invariant.

It follows that

$$
\varpi^{*}(\varphi \otimes 1): M_{0} \stackrel{m \mapsto m}{\longrightarrow} \tilde{\varpi}^{*} \varphi^{*}\left(M_{R}\right) \rightarrow \tilde{\varpi}^{*} M_{R}=M_{0} \otimes_{W} \widehat{S}
$$

\footnotetext{
${ }^{8} \mathrm{By}$ the main result of $\left[\mathrm{Me}\right.$. Note that the divided powers on $\mathrm{Fil}^{1} S$ are not topologically nilpotent, so we cannot make the following argument with $S$ in place of $\widehat{S}$, as the theorem in $\mathrm{Me}$ does not apply. When $\mathscr{G}_{0}^{*}$ is connected the main result of loc. cit should hold without assuming the divided powers are nilpotent, but as remarked in [Zi 1, p. 214] there seems to be no reference for this.
} 
has the form $m \mapsto A \varphi(m)$ for some $A \in U_{G}^{\circ}(\widehat{S})$. This means that $\tilde{\varpi}$ factors through $R_{G}$, and hence so does $\varpi$.

Finally suppose that $\mathscr{G}_{0}^{*}$ is connected. Then using results of Zink, we can repeat the above argument with $S$ in place of $\widehat{S}$, even when $p=2$ : Consider the map $S \rightarrow \mathcal{O}_{K} \times_{k} W$ sending $u$ to $(\pi, 0)$, and choose a $G_{S}$-split filtration on $\mathbb{D}\left(\mathscr{G}_{\varpi}\right)(S)$ which lifts the filtrations on $\mathbb{D}(\mathscr{G})(W)$ and $\mathbb{D}\left(\mathscr{G}_{\varpi}\right)\left(\mathcal{O}_{K}\right)$. In the terminology of [Zi 2 ] this filtration gives $\mathbb{D}\left(\mathscr{G}_{\varpi}\right)(S)$ the structure of an $S$-window over $S$, and hence gives rise to a $p$-divisible group $\mathscr{G}_{\tilde{\varpi}}$ over $S$ which deforms $\mathscr{G}_{\varpi \times \varpi_{0}}$. By [Zi 1, Cor. 97] the canonical isomorphism $\mathbb{D}\left(\mathscr{G}_{\tilde{\varpi}}\right)(S) \stackrel{\sim}{\longrightarrow} \mathbb{D}\left(\mathscr{G}_{\varpi}\right)(S)$ respects filtrations. The rest of the argument is as in the case $p>2$.

Corollary (1.5.11). Suppose $p>2$ or $\mathscr{G}_{0}^{*}$ is connected. Let $K^{\prime} / K$ be a finite extension and $\varpi: R \rightarrow \mathcal{O}_{K^{\prime}}$ a map of $W$-algebras inducing a p-divisible group $\mathscr{G}_{\varpi}$ over $\mathcal{O}_{K^{\prime}}$. Let $L=T_{p} \mathscr{G}^{*}$ and suppose there exists a family of $G_{K^{\prime}}$-invariant tensors $\left(s_{\alpha, \text { ét }}\right) \subset L^{\otimes}$ defining a reductive subgroup of $\mathrm{GL}(L)$, such that under the $p$-adic comparison isomorphism

$$
L \otimes_{\mathbb{Z}_{p}} B_{\text {cris }} \stackrel{\sim}{\longrightarrow} M_{0} \otimes_{\mathbb{Z}_{p}} B_{\text {cris }},
$$

$s_{\alpha, \text { ét }}$ maps to $s_{\alpha} \in M_{0}^{\otimes}$.

Then $\varpi$ factors through $R_{G}$.

Proof. Let $k^{\prime}$ be the residue field of $K^{\prime}$. After replacing $R$ and $R_{G}$ by $R \otimes_{W} W\left(k^{\prime}\right)$ and $R \otimes_{W} W\left(k^{\prime}\right)$, respectively, we may assume that $K^{\prime}=K$. We use the notation

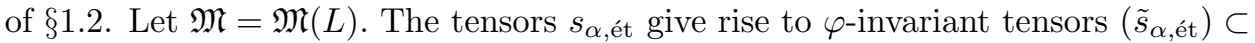
$\mathfrak{M}^{\otimes}$, which define a reductive subgroup of GL( $(\mathfrak{M})$. Then we have

$$
\left(1 \otimes \tilde{s}_{\alpha, \text { ét }}\right) \subset \mathcal{M}(\mathfrak{M}) \stackrel{\sim}{\longrightarrow} \mathbb{D}(\mathscr{G})(S),
$$

by (1.4.2). We saw in the proof of (1.3.6) that the image of $s_{\alpha \text {,ét }}$ under the $p$-adic comparison isomorphism is $\left.\tilde{s}_{\alpha \text {,ét }}\right|_{u=0}$. Hence $\left.1 \otimes \tilde{s}_{\alpha \text {,ét }}\right|_{u=0}=s_{\alpha}$ and $\left(1 \otimes \tilde{s}_{\alpha \text {,ét }}\right) \subset$ $\mathbb{D}(\mathscr{G})(S)$ is a collection of tensors satisfying the hypotheses of (1.5.8).

\section{Integral CANonical models of Hodge type}

(2.1) Shimura varieties. We recall the definition of a Shimura datum and the associated Shimura variety [De 3, §2.1]. Let $G$ be a connected reductive group over $\mathbb{Q}$ and $X$ a conjugacy class of maps of algebraic groups over $\mathbb{R}$,

$$
h: \mathbb{S}=\operatorname{Res}_{\mathbb{C} / \mathbb{R}} \mathbb{G}_{m} \rightarrow G_{\mathbb{R}} .
$$

On $\mathbb{R}$-points such a map induces a map of real groups $\mathbb{C}^{\times} \rightarrow G(\mathbb{R})$.

We require that $(G, X)$ satisfy the following conditions:

(1) Let $\mathfrak{g}$ denote the Lie algebra of $G_{\mathbb{R}}$. We require that the composite

$$
\mathbb{S} \rightarrow G_{\mathbb{R}} \rightarrow G_{\mathbb{R}}^{\mathrm{ad}} \rightarrow \mathrm{GL}(\mathfrak{g})
$$

defines a Hodge structure of type $(-1,1),(0,0),(1,-1)$. This means that under the action of $\mathbb{C}^{\times}$on $\mathfrak{g}_{\mathbb{C}}=\mathfrak{g} \otimes_{\mathbb{R}} \mathbb{C}$ we have a decomposition

$$
\mathfrak{g}_{\mathbb{C}}=V^{-1,1} \oplus V^{0,0} \oplus V^{1,-1},
$$

where $z \in \mathbb{C}^{\times}$acts on $V^{p, q}$ via $z^{-p} \bar{z}^{-q}$.

(2) $h(i)$ is a Cartan involution of $G_{\mathbb{R}}^{\text {ad }}$ (note that $\operatorname{ad} h(-1)=1$ on $\mathfrak{g}$, so $h$ induces an involution of $\left.G_{\mathbb{R}}^{\text {ad }}\right)$. This means that we require the real form of $G^{\text {ad }}$ defined by the involution $g \mapsto h(i) \bar{g} h(i)^{-1}$ to be compact. 
(3) $G^{\text {ad }}$ has no factor defined over $\mathbb{Q}$ whose real points form a compact group.

The second condition implies that for any $h_{0} \in X$ the stabilizer $K_{\infty} \subset G(\mathbb{R})$ (acting by conjugation) of $h_{0}$ is compact modulo its center, and $G(\mathbb{R}) / K_{\infty} \stackrel{\sim}{\longrightarrow} X$ has a complex structure. A pair $(G, X)$ satisfying the above conditions is called a Shimura datum.

Let $\mathbb{A}_{f}$ denote the finite adeles over $\mathbb{Q}$, and $\mathbb{A}_{f}^{p} \subset \mathbb{A}_{f}$ the subgroup of adeles with trivial component at a prime $p$. Let $K=K_{p} K^{p} \subset G\left(\mathbb{A}_{f}\right)$ be a compact open subgroup, where $K_{p} \subset G\left(\mathbb{Q}_{p}\right)$, and $K^{p} \subset G\left(\mathbb{A}_{f}^{p}\right)$ are compact open.

A theorem of Baily-Borel asserts that

$$
\operatorname{Sh}_{K}(G, X)_{\mathbb{C}}=G(\mathbb{Q}) \backslash X \times G\left(\mathbb{A}_{f}\right) / K
$$

has a natural structure of an algebraic variety over $\mathbb{C}$. Results of Shimura, Deligne, Milne and others imply that $\operatorname{Sh}_{K}(G, X)_{\mathbb{C}}$ has a model $\operatorname{Sh}_{K}(G, X)$ over a number field $E=E(G, X)$, the reflex field, which does not depend on $K$ [Mi 2, $, 44,5]$.

We will sometimes consider the pro-variety

$$
\operatorname{Sh}_{K_{p}}(G, X)=\operatorname{limSh}_{K}(G, X)
$$

where $K$ runs through compact open subgroups as above with a fixed factor $K_{p}$ at $p$. Similarly we denote by $\operatorname{Sh}(G, X)$ the projective limit taken over all compact open subgroups $K \subset G\left(\mathbb{A}_{f}\right)$.

A morphism $i:\left(G_{1}, X_{1}\right) \rightarrow\left(G_{2}, X_{2}\right)$ of Shimura data is a map of groups $G_{1} \rightarrow$ $G_{2}$, which induces a map $X_{1} \rightarrow X_{2}$. A morphism $i$ induces a map

$$
\operatorname{Sh}_{K_{1}}\left(G_{1}, X_{1}\right) \rightarrow \operatorname{Sh}_{K_{2}}\left(G_{2}, X_{2}\right),
$$

provided the compact open subgroups are chosen so that $K_{1}$ maps into $K_{2}$. This map is defined over the composite of the reflex fields $E\left(G_{1}, X_{1}\right) \cdot E\left(G_{2}, X_{2}\right)$ [De 1 , 5.4]. If $i$ is an embedding, then for any $K_{1}$, the subgroup $K_{2}$ can always be chosen so that (2.1.1) is a closed embedding [De 1, 1.15]. We will need the following refinement of this result.

Lemma (2.1.2). Let $i:\left(G_{1}, X_{1}\right) \hookrightarrow\left(G_{2}, X_{2}\right)$ be an embedding of Shimura data, and $K_{2, p} \subset G_{2}\left(\mathbb{Q}_{p}\right)$ a compact open subgroup. Let $K_{1}=K_{1, p} K_{1}^{p}$ be a compact open subgroup of $G_{1}\left(\mathbb{A}_{f}\right)$ such that $K_{1, p}=K_{2, p} \cap G\left(\mathbb{Q}_{p}\right)$. Then there exists a compact open subgroup $K_{2}=K_{2, p} K_{2}^{p}$ of $G_{2}\left(\mathbb{A}_{f}\right)$ with $K_{1} \subset K_{2}$, and such that $i$ induces an embedding

$$
\operatorname{Sh}_{K_{1}}\left(G_{1}, X_{1}\right) \hookrightarrow \operatorname{Sh}_{K_{2}}\left(G_{2}, X_{2}\right) .
$$

Proof. We follow the argument of [De 1, 15], which shows that it suffices to check that the map

$$
\operatorname{Sh}_{K_{1, p}}\left(G_{1}, X_{1}\right) \rightarrow \operatorname{Sh}_{K_{2, p}}\left(G_{2}, X_{2}\right)
$$

is an embedding. We now compute the complex points of these Shimura varieties.

Let $Z_{1} \subset G_{1}$ and $Z_{2} \subset G_{2}$ denote the centers of $G_{1}$ and $G_{2}$. Write $Z_{1}(\mathbb{Q})^{-}$and $Z_{2}(\mathbb{Q})^{-}$for the closures of $Z_{1}(\mathbb{Q})$ and $Z_{2}(\mathbb{Q})$ in $G_{1}\left(\mathbb{A}_{f}^{p}\right)$ and $G_{2}\left(\mathbb{A}_{f}^{p}\right)$, respectively. Let $U_{1} \subset Z_{1}(\mathbb{Q})$ be the group of units 9 By a theorem of Chevalley $\mathrm{Ch}$ any finite

\footnotetext{
${ }^{9}$ An element $u \in Z_{1}(\mathbb{Q})$ is a unit if the sequence $\left\{u^{n}\right\}_{n>1}$ is contained in a compact subset of $Z_{1}\left(\mathbb{Q}_{p}\right)$ for any prime $p$. If we embed $Z_{1}$ into a torus of the form $\prod_{i} \operatorname{Res}_{F_{i} / \mathbb{Q}} \mathbb{G}_{m}$ with $F_{i}$ a number field, then this condition holds if and only if the image of $u$ in each factor $F_{i}^{\times}$is a unit in the number field $F_{i}$.
} 
index subgroup of $U_{1}$ is open in the topology induced by the inclusion $U_{1} \subset Z_{1}\left(\mathbb{A}_{f}^{p}\right)$. Hence

$$
Z_{1}(\mathbb{Q})^{-}=\lim _{n} Z_{1}(\mathbb{Q}) / U_{1}^{n}
$$

and $Z_{1}(\mathbb{Q})^{-}$is also equal to the closure of $Z_{1}(\mathbb{Q})$ in $G_{1}\left(\mathbb{A}_{f}\right)$. We set

$$
\widehat{G}_{1}(\mathbb{Q})=\lim _{n} G_{1}(\mathbb{Q}) / U_{1}^{n},
$$

and similarly for $\widehat{G}_{2}(\mathbb{Q})$. Then $\widehat{G}_{1}(\mathbb{Q})$ is an extension of $G_{1}(\mathbb{Q}) / Z_{1}(\mathbb{Q})$ by $Z_{1}(\mathbb{Q})^{-}$. One checks easily that $\widehat{G}_{1}(\mathbb{Q})$ injects into $G_{1}\left(\mathbb{A}_{f}^{p}\right)$. Now the complex points of $\operatorname{Sh}_{K_{1}}\left(G_{1}, X_{1}\right)$ are given by

$$
G_{1}(\mathbb{Q}) \backslash X_{1} \times G\left(\mathbb{A}_{f}\right) / K_{1}=\left(G_{1}(\mathbb{Q}) / Z_{1}(\mathbb{Q})\right) \backslash X_{1} \times\left(G_{1}\left(\mathbb{A}_{f}\right) / Z_{1}(\mathbb{Q}) \cdot K_{1}\right) .
$$

Passing to the inverse limit with $K_{1}^{p}$, we see that $\operatorname{Sh}_{K_{1, p}}\left(G_{1}, X_{1}\right)(\mathbb{C})$ is given by (cf. [De 3, 2.1.10])

$$
\left(G_{1}(\mathbb{Q}) / Z_{1}(\mathbb{Q})\right) \backslash X_{1} \times\left(G_{1}\left(\mathbb{A}_{f}\right) / Z_{1}(\mathbb{Q})^{-} \cdot K_{1, p}\right)=\widehat{G}_{1}(\mathbb{Q}) \backslash X_{1} \times G_{1}\left(\mathbb{A}_{f}\right) / K_{1, p} .
$$

To show that

$$
\widehat{G}_{1}(\mathbb{Q}) \backslash X_{1} \times G_{1}\left(\mathbb{A}_{f}\right) / K_{1, p} \rightarrow \widehat{G}_{2}(\mathbb{Q}) \backslash X_{2} \times G_{1}\left(\mathbb{A}_{f}\right) / K_{2, p}
$$

is an injection, consider the map

$$
\widehat{G}_{1}(\mathbb{Q}) \backslash G_{1}\left(\mathbb{A}_{f}^{p}\right) \rightarrow \widehat{G}_{2}(\mathbb{Q}) \backslash G_{2}\left(\mathbb{A}_{f}^{p}\right) .
$$

Note that for any $x \in X_{1}$, the image of $Z_{1}(\mathbb{Q})$ in $G_{2}(\mathbb{Q})$ is contained in the centralizer of $\mathbb{S} \stackrel{h_{x}}{\rightarrow} G_{1}(\mathbb{R}) \rightarrow G_{2}(\mathbb{R})$, which is compact mod center. Hence $U_{2}$ contains a subgroup which has finite index in the image of $U_{1}$ and (2.1.4) is well defined. An argument as in De 1, 1.15.3] shows that (2.1.4) is injective. Fix a set of coset representatives of $\widehat{G}_{1}(\mathbb{Q})$ in $G_{1}\left(\mathbb{A}_{f}^{p}\right)$. Since $\widehat{G}_{1}(\mathbb{Q})$ is a subgroup of $G_{1}\left(\mathbb{A}_{f}^{p}\right)$, the fibres of the projection

$$
\widehat{G}_{1}(\mathbb{Q}) \backslash X_{1} \times G_{1}\left(\mathbb{A}_{f}\right) / K_{1, p} \rightarrow \widehat{G}_{1}(\mathbb{Q}) \backslash G_{1}\left(\mathbb{A}_{f}^{p}\right)
$$

may be identified with $X_{1} \times G_{1}\left(\mathbb{Q}_{p}\right) / K_{1, p}$. Since $K_{1, p}=K_{2, p} \cap G_{1}\left(\mathbb{Q}_{p}\right)$, the map

$$
X_{1} \times G_{1}\left(\mathbb{Q}_{p}\right) / K_{1, p} \rightarrow X_{2} \times G_{2}\left(\mathbb{Q}_{p}\right) / K_{2, p}
$$

is injective, and the lemma follows.

(2.1.5) Fix a $\mathbb{Q}$-vector space $V$ with a perfect alternating pairing $\psi$. For any $\mathbb{Q}$-algebra $R$, we write $V_{R}=V \otimes_{\mathbb{Q}} R$. Take $G=\operatorname{GSp}(V, \psi)$, the corresponding group of symplectic similitudes, and let $X=S^{ \pm}$be the Siegel double space, defined as the set of maps $h: \mathbb{S} \rightarrow G_{\mathbb{R}}$ such that:

(1) The $\mathbb{C}^{\times}$action on $V_{\mathbb{R}}$ gives rise to a Hodge structure of type $(-1,0),(0,1)$ :

$$
V_{\mathbb{C}} \stackrel{\sim}{\longrightarrow} V^{-1,0} \oplus V^{0,-1} \text {. }
$$

(2) $(x, y) \mapsto \psi(x, h(i) y)$ is (positive or negative) definite on $V_{\mathbb{R}}$.

The reflex field of $\left(\mathrm{GSp}, S^{ \pm}\right)$is $\mathbb{Q}$.

If $V_{\mathbb{Z}} \subset V$ is a $\mathbb{Z}$-lattice, and $h \in S^{ \pm}$, then $V^{-1,0} / V_{\mathbb{Z}}$ is an abelian variety. For a ring $R$ we write $V_{R}=V_{\mathbb{Z}} \otimes_{\mathbb{Z}} R$. If $V_{\widehat{\mathbb{Z}}}$ is stable by $K$, then for $K^{p}$ sufficiently small 10 this gives rise to an interpretation of $\operatorname{Sh}_{K}(G, X)=\operatorname{Sh}_{K}\left(\mathrm{GSp}, S^{ \pm}\right)$as a moduli space for abelian varieties [De 1, §4], [RZ, §6]. Here $\widehat{\mathbb{Z}}$ denotes the profinite completion

\footnotetext{
${ }^{10}$ We sometimes tacitly assume this in the following.
} 
of $\mathbb{Z}$. In particular, for sufficiently small $K^{p}$ there exists an abelian scheme $\mathcal{A}$ over $\mathrm{Sh}_{K}\left(\mathrm{GSp}, S^{ \pm}\right)$, defined over $\mathbb{Q}$.

(2.2) Absolute Hodge cycles. We recall some of Deligne's results on absolute Hodge cycles. Suppose we have an embedding of Shimura data $i:(G, X) \hookrightarrow$ (GSp, $\left.S^{ \pm}\right)$. Then $(G, X)$ is said to be of Hodge type. Fix compact open subgroups $K=K_{p} K^{p} \subset G\left(\mathbb{A}_{f}\right)$ and $K^{\prime}=K_{p}^{\prime} K^{\prime p} \subset \operatorname{GSp}\left(\mathbb{A}_{f}\right)$ such that $K \subset K^{\prime}$. Since $\left(\mathrm{GSp}, S^{ \pm}\right)$has reflex field $\mathbb{Q}$, the map $\operatorname{Sh}_{K}(G, X) \rightarrow \operatorname{Sh}_{K^{\prime}}\left(\mathrm{GSp}, S^{ \pm}\right)$induced by $i$ is defined over $E=E(G, X)$.

Let $\left(s_{\alpha, B}\right) \subset V^{\otimes}$ be a finite collection of tensors defining the subgroup $G \subset$ $\operatorname{GSp}(V) \subset \mathrm{GL}(V)$ (see (1.3.2) above). Fix a $\mathbb{Z}$-lattice $V_{\mathbb{Z}} \subset V$ such that $V_{\mathbb{Z}} \otimes_{\mathbb{Z}} \widehat{\mathbb{Z}}$ is stable by $K^{\prime}$, as in (2.1.5), and let $h: \mathcal{A} \rightarrow \operatorname{Sh}_{K}(G, X)$ denote the universal abelian scheme, which exists assuming that $K^{p}$ is sufficiently small. Write $\mathcal{V}=R^{1} h_{*} \Omega^{\bullet}$ for the first relative de Rham cohomology of $\mathcal{A}$. This is a vector bundle on $\operatorname{Sh}_{K}(G, X)$ equipped with an integrable connection $\nabla$. Let $\tilde{\mathcal{V}}_{\mathbb{C}}$ and $\mathcal{V}_{\mathbb{C}}$ denote the pullbacks of $\mathcal{V}$ to $X \times G\left(\mathbb{A}_{f}\right) / K$ and $\operatorname{Sh}_{K}(G, X)_{\mathbb{C}}$, respectively, so that $\tilde{\mathcal{V}}_{\mathbb{C}}$ is an analytic vector bundle over a complex analytic space.

Using the de Rham isomorphism, we may view $s_{\alpha, B}$ as a section of $\tilde{\mathcal{V}}_{\mathbb{C}}^{\otimes}$. Since $s_{\alpha, B}$ is $G(\mathbb{Q})$-equivariant, this section descends to a section $s_{\alpha, \mathrm{dR}}$ of $\mathcal{V}_{\mathbb{C}}^{\otimes}$. More precisely, $s_{\alpha, B}$ descends to a $\nabla$-parallel section of $\mathcal{V}_{\mathbb{C}}^{\text {an } \otimes}$, where $\mathcal{V}_{\mathbb{C}}^{\text {an }}$ is the analytic vector bundle attached to $\mathcal{V}_{\mathbb{C}}$. Any $\nabla$-parallel section of $\mathcal{V}_{\mathbb{C}}^{\text {an } \otimes}$ arises from $\mathcal{V}_{\mathbb{C}}^{\otimes}$. This follows from the equivalence between algebraic vector bundles equipped with a flat connection with regular singular points, and the category of analytic vector bundles equipped with a flat connection De 4] (cf. De 2, p. 31]).

Now let $\kappa \supset E$ be a field of characteristic 0 , and $\bar{\kappa}$ an algebraic closure of $\kappa$. Fix an embedding $\mathbb{Q}_{p} \hookrightarrow \mathbb{C}$ and an embedding of $E$-algebras $\sigma: \bar{\kappa} \hookrightarrow \mathbb{C}$. Let $x \in \operatorname{Sh}_{K}(G, X)(\kappa)$ and denote by $\mathcal{A}_{x}$ the corresponding abelian variety over $\kappa$. Denote by $H_{B}^{1}\left(\mathcal{A}_{x}(\mathbb{C}), \mathbb{Q}\right)$ the Betti cohomology of $\mathcal{A}_{x}(\mathbb{C})$. Write $H_{\mathrm{dR}}^{1}\left(\mathcal{A}_{x}\right)$ for its de Rham cohomology and $H_{\text {êt }}^{1}\left(\mathcal{A}_{x, \bar{\kappa}}\right)=H_{\text {êt }}^{1}\left(\mathcal{A}_{x, \bar{\kappa}}, \mathbb{Q}_{p}\right)$ for the $p$-adic étale cohomology of $\mathcal{A}_{x, \bar{\kappa}}=\mathcal{A}_{x} \otimes_{\kappa} \bar{\kappa}$. The embedding $\sigma$ induces isomorphisms

$$
H_{\mathrm{dR}}^{1}\left(\mathcal{A}_{x}\right) \otimes_{\kappa, \sigma} \mathbb{C} \stackrel{\sim}{\longrightarrow} H_{B}^{1}\left(\mathcal{A}_{x}(\mathbb{C}), \mathbb{Q}\right) \otimes_{\mathbb{Q}} \mathbb{C} \stackrel{\sim}{\longrightarrow} H^{1}\left(\mathcal{A}_{x, \bar{\kappa}}, \mathbb{Q}_{p}\right) \otimes_{\mathbb{Q}_{p}} \mathbb{C} .
$$

Let $s_{\alpha, B, x}$ be the fibre of $s_{\alpha, B}$ at $x$ (regarded as a $\mathbb{C}$-valued point via $\sigma$ ), and denote by $s_{\alpha, \mathrm{dR}, x} \in H_{\mathrm{dR}}^{1}\left(\mathcal{A}_{x}\right)^{\otimes} \otimes_{\kappa, \sigma} \mathbb{C}$ and $s_{\alpha, \text { ét }, x} \in H_{\text {êt }}^{1}\left(\mathcal{A}_{x, \bar{\kappa}}\right)^{\otimes}$ the images of $s_{\alpha, B, x}$ under these two isomorphisms.

Lemma (2.2.1). The action of $\operatorname{Gal}(\bar{\kappa} / \kappa)$ on $H_{\text {ét }}^{1}\left(\mathcal{A}_{x, \bar{\kappa}}, \mathbb{Q}_{p}\right)$ fixes each $s_{\alpha, \text { ét }, x}$ and factors through $G\left(\mathbb{Q}_{p}\right)$. Moreover we have $s_{\alpha, \mathrm{dR}, x} \in H_{\mathrm{dR}}^{1}\left(\mathcal{A}_{x}\right)^{\otimes}$.

Proof. Let $\operatorname{Sh}_{K^{p}}(G, X)=\lim _{H_{p}} \operatorname{Sh}_{H_{p} K^{p}}(G, X)$, where $H_{p}$ runs over compact open subgroups of $K_{p}$, and similarly for $\operatorname{Sh}_{K^{\prime} p}\left(\mathrm{GSp}, S^{ \pm}\right)$.

The action of $\operatorname{Gal}(\bar{\kappa} / \kappa)$ on $H_{\text {ét }}^{1}\left(\mathcal{A}_{x, \bar{\kappa}}, \mathbb{Q}_{p}\right)$ is induced by the map $\operatorname{Gal}(\bar{\kappa} / \kappa) \rightarrow K_{p}^{\prime}$, obtained by pulling back to $\bar{\kappa}$ the $K_{p}^{\prime}$-torsor $\operatorname{Sh}_{K^{\prime p}}\left(\mathrm{GSp}, S^{ \pm}\right) \rightarrow \mathrm{Sh}_{K^{\prime}}\left(\mathrm{GSp}, S^{ \pm}\right)$. On the other hand, we have a commutative, $K_{p}$-equivariant diagram

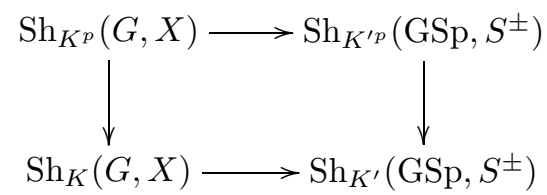


which shows that the restriction of $\operatorname{Sh}_{K^{\prime} p}\left(\mathrm{GSp}, S^{ \pm}\right)$to $\operatorname{Sh}_{K}(G, X)$ descends to a $K_{p}$-torsor. This shows that the action of $\operatorname{Gal}(\bar{\kappa} / \kappa)$ on $H_{\text {êt }}^{1}\left(\mathcal{A}_{x, \bar{\kappa}}, \mathbb{Q}_{p}\right)$ is induced by a map $\operatorname{Gal}(\bar{\kappa} / \kappa) \rightarrow K_{p} \subset G\left(\mathbb{Q}_{p}\right)$. In particular this action fixes each $s_{\alpha, \text { ét, } x}$.

To see the final statement note that, by a result of Deligne [De 2, 2.11], the Hodge cycle $\left(s_{\alpha, \mathrm{dR}, x}, s_{\alpha \text {,ét }, x}\right)$ is an absolute Hodge cycle, for each $\alpha$. In particular, this implies [De 2, 2.7] that $s_{\alpha, \mathrm{dR}, x} \in H_{\mathrm{dR}}^{1}\left(\mathcal{A}_{x}\right)^{\otimes} \otimes_{\kappa} \bar{\kappa}$. Moreover, since an absolute Hodge cycle is determined by either its de Rham or étale component, $\operatorname{Gal}(\bar{\kappa} / \kappa)$ fixes $s_{\alpha, \mathrm{dR}, x}$ as it fixes $s_{\alpha, \text { ét }, x}$. Hence $s_{\alpha, \mathrm{dR}, x} \in H_{\mathrm{dR}}^{1}\left(\mathcal{A}_{x}\right)^{\otimes}$.

Corollary (2.2.2). The section $s_{\alpha . \mathrm{dR}}$ of $\mathcal{V}^{\otimes}$ is defined over $E$, and not just over $\mathbb{C}$.

Proof. This follows by applying (2.2.1) with $\kappa$ the generic point of each component of $\operatorname{Sh}_{K}(G, X)$ (equipped with any complex embedding of $\kappa$ ).

(2.2.3) Our main results will concern the situation where $K_{p}$ is a hyperspecial subgroup of $G\left(\mathbb{Q}_{p}\right)$. Recall that this means that there is a reductive group scheme $G_{\mathbb{Z}_{p}}$ over $\mathbb{Z}_{p}$ with generic fibre $G_{\mathbb{Q}_{p}}=G \otimes_{\mathbb{Q}} \mathbb{Q}_{p}$ such that $K_{p}=G_{\mathbb{Z}_{p}}\left(\mathbb{Z}_{p}\right) \subset G\left(\mathbb{Q}_{p}\right)$. Any such subgroup is maximal compact in $G\left(\mathbb{Q}_{p}\right)$ [Ti, 1.10, 3.8].

Hyperspecial subgroups exist if and only if $G_{\mathbb{Q}_{p}}$ is quasi-split and split over an unramified extension. Under these conditions the reflex field $E=E(G, X)$ is unramified at $p$ [Mi 1, 4, 4.7]. For the rest of this section we assume that $K_{p}$ is hyperspecial.

Fix an algebraic closure $\bar{E}$ of $E$, and let $\pi_{0}\left(\operatorname{Sh}_{K}(G, X)\right)$ denote the set of connected components of $\operatorname{Sh}_{K}(G, X) \otimes_{E} \bar{E}$. We refer to these as the geometrically connected components of $\operatorname{Sh}_{K}(G, X)$.

Proposition (2.2.4). The geometrically connected components of $\operatorname{Sh}_{K}(G, X)$ are defined over an extension of $E$ which is unramified at primes dividing $p$.

Proof. One can extract this from Deligne's description of the action of $\operatorname{Gal}(\bar{E} / E)$ on the geometrically connected components De 3, 2.6.3]. We give a more direct argument, though still based on the reciprocity law for special points.

Let

$$
\pi_{0}\left(\operatorname{Sh}_{K_{p}}(G, X)\right)=\lim _{K^{p}} \pi_{0}\left(\operatorname{Sh}_{K^{p} K_{p}}(G, X)\right) .
$$

By (2.2.5) below, $G\left(\mathbb{A}_{f}^{p}\right)$ acts transitively on $\pi_{0}\left(\operatorname{Sh}_{K_{p}}(G, X)\right)$. As this action commutes with that of $\operatorname{Gal}(\bar{E} / E)$, it suffices to exhibit an element of $\pi_{0}\left(\operatorname{Sh}_{K_{p}}(G, X)\right)$ whose stabilizer in $\operatorname{Gal}(\bar{E} / E)$ corresponds to an (infinite) extension of $E$ which is unramified at each $v \mid p$. We will do this by showing the stronger statement that there is a point of $\operatorname{Sh}_{K_{p}}(G, X)$ defined over such an extension. The argument is a variant of that of $[\mathrm{De} 2$, p. 75].

Let $x_{\infty} \in \operatorname{Sh}_{K}(G, X)(\mathbb{C})$ be any point, and $T_{\infty} \subset G_{\mathbb{R}}$ a maximal torus containing the image of the cocharacter $h_{x_{\infty}}: \mathbb{S} \rightarrow G_{\mathbb{R}}$ corresponding to $x_{\infty}$. Let $\lambda_{\infty} \in \operatorname{Lie} T_{\infty}$ be a regular element such that $T_{\infty}$ is the centralizer of $\lambda_{\infty}$ in $G_{\mathbb{R}}$. Let $T_{p} \subset G_{\mathbb{Q}_{p}}$ be a maximal torus which is the generic fibre of a maximal torus $T_{p, \mathbb{Z}_{p}} \subset G_{\mathbb{Z}_{p}}$. Then $T_{p}$ splits over an unramified extension of $\mathbb{Q}_{p}$. Let $\lambda_{p} \in \operatorname{Lie} T_{p}$ be a regular element so that $T_{p}$ is the centralizer of $\lambda_{p}$ in $G_{\mathbb{Q}_{p}}$.

Since the map $h \mapsto\left[h, \lambda_{p}\right]$ induces an automorphism of Lie $G_{\mathbb{Q}_{p}} / \operatorname{Lie} T_{p}$, the image of the map

$$
G\left(\mathbb{Q}_{p}\right) \rightarrow \operatorname{Lie} G_{\mathbb{Q}_{p}} / \operatorname{Lie} T_{p} ; \quad g \mapsto \operatorname{ad}(g)\left(\lambda_{p}\right)
$$


contains an open neighbourhood of 0 . Hence, if $\lambda \in \operatorname{Lie} G$ is a regular element which is sufficiently close to $\lambda_{\infty}$ in the real vector space Lie $G_{\mathbb{R}}$ and to $\lambda_{p}$ in the $\mathbb{Q}_{p}$-vector space Lie $G_{\mathbb{Q}_{p}}$, then there exists $g_{p} \in G_{\mathbb{Z}_{p}}\left(\mathbb{Z}_{p}\right)$ such that $\operatorname{ad}\left(g_{p}\right)(\lambda) \in \operatorname{Lie} T_{p}$ and similarly, there exists $g_{\infty} \in G(\mathbb{R})^{+}$, the connected component of the identity in $G(\mathbb{R})$, such that $\operatorname{ad}\left(g_{\infty}\right)(\lambda) \in \operatorname{Lie} T_{\infty}$. Thus, if $T \subset G$ is the centralizer of $\lambda$, then

$$
T=g_{p}^{-1} T_{p} g_{p}=g_{\infty}^{-1} T_{\infty} g_{\infty} .
$$

The cocharacter $h=\operatorname{ad}\left(g_{\infty}^{-1}\right)\left(h_{x_{\infty}}\right)$ factors through $T_{\mathbb{R}}$. Let $J_{p}=T\left(\mathbb{Q}_{p}\right) \cap K_{p}=$ $g_{p}^{-1} T_{p, \mathbb{Z}_{p}} g_{p}\left(\mathbb{Z}_{p}\right), J^{p}=T\left(\mathbb{A}_{f}^{p}\right) \cap K^{p}$ and set $J=J_{p} J^{p}$. The reflex field $E(T, h)$ is contained in the splitting field of $T$, which is unramified over $p$. The points of $\operatorname{Sh}_{J}(T, h)$ are defined over the abelian extension of $E(T, h)$ corresponding to the quotient $T\left(\mathbb{A}_{f}\right) / T(\mathbb{Q}) T(\mathbb{R})^{+} J$, where $T(\mathbb{R})^{+} \subset T(\mathbb{R})$ denotes the connected component of the identity [De 3, 2.6]. Since $J_{p} \subset T\left(\mathbb{Q}_{p}\right)$ is maximal compact this is an extension of $E(T, h)$ which is unramified at any prime over $p$. It follows that any point in the image of

$$
\mathrm{Sh}_{J_{p}}(T, h) \rightarrow \mathrm{Sh}_{K_{p}}(G, X)
$$

is defined over an extension of $E$ in which $p$ is unramified.

Lemma (2.2.5). $G\left(\mathbb{A}_{f}^{p}\right)$ acts transitively on $\pi_{0}\left(\operatorname{Sh}_{K_{p}}(G, X)\right)$.

Proof. Fix a cocharacter $h_{0} \in X$, so that $X \stackrel{\sim}{\longrightarrow} G(\mathbb{R}) / K_{\infty}$ as in (2.1). Let $x \in$ $\mathrm{Sh}_{K_{p}}(G, X)$ and $\left[h, g_{p}, g^{p}\right]$ be a representative of $x$ where $h \in G(\mathbb{R}), g_{p} \in G\left(\mathbb{Q}_{p}\right)$ and $g^{p} \in G\left(\mathbb{A}_{f}^{p}\right)$. We have to show that we can choose this representative so that $h \in G(\mathbb{R})^{+}$and $g_{p} \in K_{p}$.

By the real approximation theorem, $G(\mathbb{Q})$ is dense in $G(\mathbb{R})$, so we may assume $h \in G(\mathbb{R})^{+}$. By $(2.2 .6)$ below, we may write $g_{p}=g_{0} g_{p}^{\prime}$ with $g_{0} \in G(\mathbb{Q})^{+}=G(\mathbb{Q}) \cap$ $G(\mathbb{R})^{+}$and $g_{p} \in K_{p}$. Then the triple $\left[g_{0}^{-1} h, g_{p}^{\prime}, g_{0}^{-1} g^{p}\right]$ represents $x$ and has the required property.

Lemma (2.2.6). Let $H$ be a connected reductive group over $\mathbb{Z}_{(p)}$. Then we have $H\left(\mathbb{Z}_{p}\right) \cdot H(\mathbb{Q})^{+}=H\left(\mathbb{Q}_{p}\right)$, where $H(\mathbb{Q})^{+}=H(\mathbb{Q}) \cap H(\mathbb{R})^{+}$.

Proof. Suppose first that $H$ is a torus over $\mathbb{Z}_{(p)}$. Let $H(\mathbb{R})^{c} \subset H(\mathbb{R})$ denote the maximal compact subgroup. Then

$$
H(\mathbb{Q}) \cdot\left[H(\mathbb{R})^{c} \times H\left(\mathbb{Z}_{p}\right)\right]=H(\mathbb{R}) \times H\left(\mathbb{Q}_{p}\right)
$$

by a result of Colliot-Thélène and Suresh CSu, 2.1,2.2]. Hence

$$
H(\mathbb{Q})^{+} \cdot\left[H(\mathbb{R})^{c} \times H\left(\mathbb{Z}_{p}\right)\right]=H(\mathbb{R})^{+} \times H\left(\mathbb{Q}_{p}\right),
$$

and the lemma follows for $H$ a torus.

In general, let $T \subset H$ be a maximal torus such that $T_{\mathbb{Q}_{p}}$ contains a maximal split torus in $H_{\mathbb{Q}_{p}}$. Such a torus can be constructed using the same argument as in the proof of (2.2.4): Take a maximal torus $T_{p, \mathbb{Z}_{p}} \subset H_{\mathbb{Z}_{p}}$ which is the centralizer of a maximal split torus in $H_{\mathbb{Z}_{p}}=H \otimes_{\mathbb{Z}_{(p)}} \mathbb{Z}_{p}$. Let $\lambda_{p} \in$ Lie $H_{\mathbb{Z}_{p}}$ be a regular element whose centralizer in $H_{\mathbb{Z}_{p}}$ is $T_{p, \mathbb{Z}_{p}}$ and define $T$ to be the centralizer of an element $\lambda \in$ Lie $H$ which is sufficiently close to $\lambda_{p}$.

Let $\tilde{H}$ be the universal cover of $H^{\text {der }}$. Then by the strong approximation theorem, $\tilde{H}\left(\mathbb{Z}_{p}\right) \cdot \tilde{H}(\mathbb{Q})^{+}=\tilde{H}\left(\mathbb{Q}_{p}\right)$, so $H\left(\mathbb{Z}_{p}\right) \cdot H(\mathbb{Q})^{+}$contains the image of $\tilde{H}\left(\mathbb{Q}_{p}\right)$. Since $H\left(\mathbb{Q}_{p}\right) / \tilde{H}\left(\mathbb{Q}_{p}\right)$ is abelian,

$$
H\left(\mathbb{Z}_{p}\right) \cdot H(\mathbb{Q})^{+}=H\left(\mathbb{Z}_{p}\right) H(\mathbb{Q})^{+} H\left(\mathbb{Z}_{p}\right) \supset H\left(\mathbb{Z}_{p}\right) T\left(\mathbb{Q}_{p}\right) H\left(\mathbb{Z}_{p}\right)=H\left(\mathbb{Q}_{p}\right),
$$


where the inclusion follows from the case of a torus considered above, and the final equality follows from the Cartan decomposition [Ti, 3.3.3].

(2.3) Integral models. We retain the notation introduced above, so that $i$ : $(G, X) \hookrightarrow\left(\mathrm{GSp}, S^{ \pm}\right)$, and $K=K_{p} K^{p}$ with $K_{p} \subset G\left(\mathbb{Q}_{p}\right)$ hyperspecial and equal to $G_{\mathbb{Z}_{p}}\left(\mathbb{Z}_{p}\right)$ for a reductive group $G_{\mathbb{Z}_{p}}$ over $\mathbb{Z}_{p}$. We will need the following.

Lemma (2.3.1). Let $W$ be a $\mathbb{Q}_{p}$-vector space and $i: G_{\mathbb{Q}_{p}} \hookrightarrow \mathrm{GL}(W)$ a closed embedding of algebraic groups. If $p=2$ assume that $G_{\mathbb{Q}_{p}}^{\text {ad }}$ has no factors of type $B 11$ Suppose that $G_{\mathbb{Z}_{p}}$ is a reductive group over $\mathbb{Z}_{p}$ with generic fibre $G_{\mathbb{Q}_{p}}$.

Then there exists a $\mathbb{Z}_{p}$-lattice $W_{\mathbb{Z}_{p}} \subset W$ such that $i$ is induced by a closed embedding $i_{\mathbb{Z}_{p}}: G_{\mathbb{Z}_{p}} \hookrightarrow \operatorname{GL}\left(W_{\mathbb{Z}_{p}}\right)$.

Proof. Let $\mathbb{Z}_{p}^{\text {ur }}$ denote a strict henselisation of $\mathbb{Z}_{p}$, and write $\mathbb{Q}_{p}^{\text {ur }}=\mathbb{Z}_{p}^{\text {ur }}[1 / p]$. Write $W^{\text {ur }}=W \otimes_{\mathbb{Z}_{p}} \mathbb{Z}_{p}^{\text {ur }}$ and $G_{\mathbb{Z}_{p}^{\text {ur }}}=G_{\mathbb{Z}_{p}} \otimes_{\mathbb{Z}_{p}} \mathbb{Z}_{p}^{\text {ur }}$. Then $G_{\mathbb{Z}_{p}}\left(\mathbb{Z}_{p}^{\text {ur }}\right)$ is a bounded subgroup of $G_{\mathbb{Q}_{p}}\left(\mathbb{Q}_{p}^{\text {ur }}\right)$ in the sense that any regular function on $G_{\mathbb{Z}_{p}^{\text {ur }}}^{\text {is bounded on } G_{\mathbb{Z}_{p}^{\text {ur }}}}$ ( $\left.\mathbb{Z}_{p}^{\text {ur }}\right)$ Ti, $3.2,3.4,3.8]$.

Let $L \subset W^{\text {ur }}$ be any $\mathbb{Z}_{p}^{\text {ur }}$-lattice. The translates $g \cdot L$ for $g \in G_{\mathbb{Z}_{p}^{\text {ur }}}\left(\mathbb{Z}_{p}^{\text {ur }}\right)$ are contained in a common $\mathbb{Z}_{p}^{\text {ur }}$-lattice in $W^{\text {ur }}$. Indeed, otherwise there exists $v \in L$ and a linear form $\lambda: W^{\text {ur }} \rightarrow \mathbb{Q}_{p}^{\text {ur }}$ such that the function $g \mapsto \lambda(g \cdot v)$ is unbounded, which contradicts the boundedness of $G_{\mathbb{Z}_{p}^{\text {ur }}}\left(\mathbb{Z}_{p}^{\text {ur }}\right)$.

Let $\Gamma=\operatorname{Gal}\left(\mathbb{Q}_{p}^{\text {ur }} / \mathbb{Q}_{p}\right)$. Since $\Gamma$ is a compact group, the above shows that the translates $\gamma \cdot L$ for $\gamma \in G_{\mathbb{Z}_{p}^{\text {ur }}}\left(\mathbb{Z}_{p}^{\text {ur }}\right) \rtimes \Gamma$ are contained in a common $\mathbb{Z}_{p}^{\text {ur }}$-lattice. Hence

$$
W_{\mathbb{Z}_{p}^{\text {ur }}}=\sum_{\gamma} \gamma \cdot L
$$

is a lattice in $W^{\text {ur }}$, where $\gamma$ runs over elements of $G_{\mathbb{Z}_{p}^{\text {ur }}}\left(\mathbb{Z}_{p}^{\text {ur }}\right) \rtimes \Gamma$. Since $W_{\mathbb{Z}_{p}^{\text {ur }}}$ is $G_{\mathbb{Z}_{p}^{\text {ur }}}$-stable, $i$ induces a map of algebraic groups over $\mathbb{Z}_{p}^{\text {ur }}$,

$$
i_{\mathbb{Z}_{p}^{\text {ur }}}: G_{\mathbb{Z}_{p}^{\text {ur }}} \rightarrow \mathrm{GL}\left(W_{\mathbb{Z}_{p}^{\text {ur }}}\right)
$$

by $[\mathrm{BT}, 1.7 .6]$. Since $W_{\mathbb{Z}_{p}^{\text {ur }}}$ is $\Gamma$-stable it arises from a $\mathbb{Z}_{p}$-lattice $W_{\mathbb{Z}_{p}} \subset W$ by étale descent. The map $i_{\mathbb{Z}_{p}^{\text {ur }}}$ is compatible with the descent data on the source and target, as this can be checked on generic fibres, so it descends to a map $i_{\mathbb{Z}_{p}}: G_{\mathbb{Z}_{p}} \rightarrow$ $\mathrm{GL}\left(W_{\mathbb{Z}_{p}}\right)$. Finally our hypotheses when $p=2$ insure that $i_{\mathbb{Z}_{p}}$ is a closed embedding as $i$ is [PY, 1.3] (cf. Va 2, 3.1.2.1]).

(2.3.2) Now assume that if $p=2$, then $G$ has no factors of type $B$. By (2.3.1) there is a lattice $V_{\mathbb{Z}} \subset V$ such that $i$ is induced by an embedding $G_{\mathbb{Z}_{p}} \hookrightarrow \operatorname{GL}\left(V_{\mathbb{Z}_{p}}\right)$. Fix such a $V_{\mathbb{Z}}$. Since $G_{\mathbb{Z}_{p}}$ has generic fibre $G \otimes_{\mathbb{Q}} \mathbb{Q}_{p}$, flat base change implies that the closure of $G$ in $\operatorname{GL}\left(V_{\mathbb{Z}_{(p)}}\right)$ is a reductive subgroup $G_{\mathbb{Z}_{(p)}}$ with $G_{\mathbb{Z}_{(p)}} \otimes_{\mathbb{Z}_{(p)}} \mathbb{Z}_{p}=G_{\mathbb{Z}_{p}}$. By (1.3.2), $G_{\mathbb{Z}_{(p)}}$ is defined by a finite collection of tensors $\left(s_{\alpha}\right) \subset V_{\mathbb{Z}_{(p)}}^{\otimes}$, which we now fix.

Since $K_{p}$ leaves $V_{\mathbb{Z}_{(p)}}$ stable by construction, $K$ leaves $V_{\widehat{\mathbb{Z}}}$ stable provided $K^{p}$ is small enough. Fix such a $K^{p}$. Let $K_{p}^{\prime} \subset \operatorname{GSp}\left(\mathbb{Q}_{p}\right)$ denote the stabilizer of $V_{\mathbb{Z}_{p}}$. This is a maximal compact subgroup of $\operatorname{GSp}\left(\mathbb{Q}_{p}\right)$ but is not, in general, hyperspecial. By (2.1.2) we may choose $K^{\prime}=K_{p}^{\prime} K^{\prime p}$ so that $i$ induces an embedding

$$
\mathrm{Sh}_{K}(G, X) \hookrightarrow \operatorname{Sh}_{K^{\prime}}\left(\mathrm{GSp}, S^{ \pm}\right) .
$$

\footnotetext{
${ }^{11}$ This restriction, which arises from the necessary restriction in the result of Prasad-Yu PY] 1.3] used in the proof, is one of the reasons for the restrictions in our results when $p=2$.
} 
After replacing $K^{\prime p}$ by an open subgroup containing $K^{p}$, we may also assume that $K^{\prime}$ leaves $V_{\widehat{\mathbb{Z}}}$ stable.

(2.3.3) Recall that the interpretation of $\mathrm{Sh}_{K^{\prime}}\left(\mathrm{GSp}, S^{ \pm}\right)$as a moduli space of polarized abelian varieties gives rise to a natural model $\mathscr{S}_{K^{\prime}}\left(\mathrm{GSp}, S^{ \pm}\right)$of this scheme over $\mathbb{Z}$. For simplicity, we describe this only over $\mathbb{Z}_{(p)}$, as this is all we will need.

After scaling the lattice $V_{\mathbb{Z}} \subset V_{\mathbb{Q}}$, we may assume that $\psi$ induces an inclusion $V_{\mathbb{Z}} \stackrel{\psi}{\rightarrow} V_{\mathbb{Z}}^{*}$ into the dual lattice $V_{\mathbb{Z}}^{*} \subset V_{\mathbb{Q}}$. Let $d=\left|V_{\mathbb{Z}}^{*} / V_{\mathbb{Z}}\right|$ and write $2 g=\operatorname{dim}_{\mathbb{Q}} V$. For a $\mathbb{Z}_{(p)}$-scheme $T$ and an abelian scheme $\mathcal{B}$ over $T$ we set

$$
\widehat{V}^{p}(\mathcal{B})=\varliminf_{p \nmid n} \mathcal{B}[n]
$$

viewed as an étale local system on $T$. Denote by $\underline{\operatorname{Isom}}\left(V_{\widehat{\mathbb{Z}}^{p}}, \widehat{V}^{p}(\mathcal{A})\right)$ the (pro-)étale sheaf on $T$ consisting of isomorphisms $V_{\widehat{\mathbb{Z}}^{p}} \stackrel{\sim}{\longrightarrow} \widehat{V}^{p}(\mathcal{A})$ which are compatible with the pairings induced by $\psi$ and $\lambda$ up to a $\widehat{\mathbb{Z}}^{p \times}$-scalar. Here $\widehat{\mathbb{Z}}^{p}=\prod_{\ell \neq p} \mathbb{Z}_{\ell}$.

We denote by $\mathcal{A}_{g, d, K^{\prime}}(T)$ the set of isomorphism classes of triples $\left(\mathcal{A}, \lambda, \varepsilon^{p}\right)$ consisting of an abelian scheme equipped with a polarization $\lambda: \mathcal{A} \rightarrow \mathcal{A}^{*}$ of degree $d$, and a section

$$
\varepsilon^{p} \in \Gamma\left(T, \underline{\operatorname{Isom}}\left(V_{\widehat{\mathbb{Z}}^{p}}, \widehat{V}^{p}(\mathcal{A})\right) / K^{p}\right) .
$$

If $K^{\prime p}$ is sufficiently small, then $\mathcal{A}_{g, d, K^{\prime}}$ is representable by a quasi-projective $\mathbb{Z}_{(p)}$-scheme, which we denote by the same symbol [MFK, Thm. 7.9]. There is a natural embedding of $\mathbb{Z}_{(p)}$-schemes $\operatorname{Sh}_{K^{\prime}}\left(\mathrm{GSp}, S^{ \pm}\right) \hookrightarrow \mathcal{A}_{g, d, K^{\prime}}$, and we define $\mathscr{S}_{K^{\prime}}\left(\mathrm{GSp}, S^{ \pm}\right)$as the closure of $\mathrm{Sh}_{K^{\prime}}\left(\mathrm{GSp}, S^{ \pm}\right)$in $\mathcal{A}_{g, d, K^{\prime}}$. For any ring $R$ we will denote by $\mathscr{S}_{K^{\prime}}\left(\mathrm{GSp}, S^{ \pm}\right)_{R}$ the base change of $\mathscr{S}_{K^{\prime}}\left(\mathrm{GSp}, S^{ \pm}\right)$to $R$.

Let $\mathcal{O}$ denote the ring of integers of $E=E(G, X)$. Let $v \mid p$ be a prime of $E$, and denote by $\mathcal{O}_{(v)}$ the localization of $\mathcal{O}$ at $v$, and by $\mathcal{O}_{v}$ the $v$-adic completion of $\mathcal{O}_{(v)}$. Denote by $\mathscr{S}_{K}^{-}(G, X)$ the closure of $\operatorname{Sh}_{K}(G, X)$ in $\mathscr{S}_{K^{\prime}}\left(\mathrm{GSp}, S^{ \pm}\right)_{\mathcal{O}_{(v)}}$.

From now on we make the following assumption when $p=2$.

(2.3.4) If $p=2$, then $G$ has no factors of type $B$, and the abelian variety over any characteristic $p$ point of $\mathscr{S}_{K}^{-}(G, X)$ has connected $p$-divisible group.

These restrictions in the results below arise from those in (1.4.3) and (2.3.1).

Proposition (2.3.5). Let $x \in \mathscr{S}_{K}^{-}(G, X)$ be a closed point with residue field of characteristic $p$, and write $\widehat{U}_{x}$ for the completion of $\mathscr{S}_{K}^{-}(G, X)$ at $x$. Then the irreducible components of $\widehat{U}_{x}$ are formally smooth over $\mathcal{O}_{(v)}$.

Proof. By (2.2.2) the tensors $s_{\alpha, \mathrm{dR}} \in \mathcal{V}^{\otimes}$ are defined over $E$, and for $F / E$ finite and $y \in \operatorname{Sh}_{K}(G, X)(F)$, the tensors $s_{\alpha, \text { ét, } y}$ are $\operatorname{Gal}(\bar{E} / F)$-invariant by (2.2.2).

Let $k$ be the residue field of $x$, write $W=W(k)$ and denote by $\mathscr{G}_{0}$ the $p$-divisible group of the abelian variety corresponding to $x$. Let $F / E$ be a finite extension and $\tilde{x} \in \mathscr{S}_{K}^{-}(G, X)(F)$ a point specializing to $x$. By (1.4.3) the $\operatorname{Gal}(\bar{E} / F)$-invariant

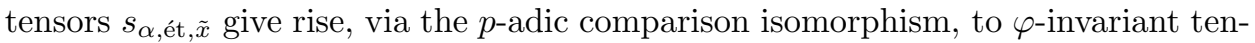
sors $\left(s_{\alpha, 0}\right) \subset \mathbb{D}\left(\mathscr{G}_{0}\right)(W)^{\otimes}$, which define a reductive subgroup $G_{W} \subset \operatorname{GL}\left(\mathbb{D}\left(\mathscr{G}_{0}\right)(W)\right)$

\footnotetext{
${ }^{12}$ One can give an explicit description of the image of this embedding and its closure as in RZ $\S 6]$, by considering triples $\left(\mathcal{A}, \lambda, \varepsilon^{p}\right)$ with $\lambda$ satisfying a more precise condition than merely being of degree $d$.
} 
such that the filtration on $\mathbb{D}\left(\mathscr{G}_{0}\right)(W) \otimes_{W} k$ is induced by a cocharacter $\mu_{0}: \mathbb{G}_{m} \rightarrow$ $G_{W} \otimes_{W} k$. Applying the theory of (1.5.4) we obtain a quotient $R_{G_{W}}$ of the versal deformation ring $R$ of $\mathscr{G}_{0}$.

Extend the valuation $v$ to $\bar{E}$. Let $\widehat{U}_{x}^{\prime}$ be the completion of $\mathscr{S}_{K^{\prime}}\left(\mathrm{GSp}, S^{ \pm}\right)$at $x$, and let $j: \widehat{U}_{x}^{\prime} \rightarrow$ Spf $R$ be a map defining the $p$-divisible group over $\widehat{U}_{x}^{\prime}$ which arises from the universal family of polarized abelian schemes over $\mathscr{S}_{K^{\prime}}\left(\mathrm{GSp}, S^{ \pm}\right)$. Then $j$ is an embedding, since a polarization on a deformation of $\mathscr{G}_{0}$ is determined by its restriction to $\mathscr{G}_{0}$. Let $Z \subset \widehat{U}_{x}$ be the irreducible component containing (the image of) $\tilde{x}$. We claim that the composite

$$
Z \hookrightarrow \widehat{U}_{x} \hookrightarrow \widehat{U}_{x}^{\prime} \hookrightarrow \operatorname{Spf} R
$$

factors through $\operatorname{Spf} R_{G_{W}}$. By (1.5.11) it suffices to check that for any finite extension $F^{\prime} / F$ in $\bar{E}$ and $\tilde{x}^{\prime} \in \operatorname{Sh}_{K}(G, X)\left(F^{\prime}\right)$ lying in $Z\left(F_{v}^{\prime}\right)$, the tensor $s_{\alpha, \text { ét, } \tilde{x}^{\prime}}$ maps to $s_{\alpha, 0}$ under the $p$-adic comparison isomorphism for the abelian variety $\mathcal{A}_{\tilde{x}^{\prime}, \bar{E}}$.

A result of Blasius and Wintenberger [B] asserts that the $p$-adic comparison isomorphism takes $s_{\alpha, \text { ét, } \tilde{x}^{\prime}}$ to $s_{\alpha, \mathrm{dR}, \tilde{x}^{\prime}}$, so it suffices to check that the isomorphism

$$
H_{\text {cris }}^{1}\left(\mathcal{A}_{x} / W\right) \otimes_{F^{\prime}} F_{v}^{\prime} \stackrel{\sim}{\longrightarrow} H_{\mathrm{dR}}^{1}\left(\mathcal{A}_{\tilde{x}^{\prime}}\right) \otimes_{F^{\prime}} F_{v}^{\prime}
$$

takes $s_{\alpha, 0}$ to $s_{\alpha, \mathrm{dR}, \tilde{x}^{\prime}}$, or equivalently that the composite

$$
H_{\mathrm{dR}}^{1}\left(\mathcal{A}_{\tilde{x}}\right) \otimes_{F} F_{v}^{\prime} \stackrel{\sim}{\longrightarrow} H_{\text {cris }}^{1}\left(\mathcal{A}_{x} / W\right) \otimes_{F^{\prime}} F_{v}^{\prime} \stackrel{\sim}{\longrightarrow} H_{\mathrm{dR}}^{1}\left(\mathcal{A}_{\tilde{x}^{\prime}}\right) \otimes_{F^{\prime}} F_{v}^{\prime}
$$

takes $s_{\alpha, \mathrm{dR}, \tilde{x}}$ to $s_{\alpha, \mathrm{dR}, \tilde{x}^{\prime}}$. Now the relative crystalline cohomology of the universal abelian variety over $\mathscr{S}_{K^{\prime}}\left(\mathrm{GSp}, S^{ \pm}\right)_{\mathcal{O}_{v}}$ is an isocrystal on $\mathscr{S}_{K^{\prime}}\left(\mathrm{GSp}, S^{ \pm}\right)_{\mathcal{O}_{v}}$, BO, $\S 2]$ and the composite in (2.3.6) is given by parallel transport with respect to the Gauss-Manin connection [BO, 2.9]. Since the generic fibre $Z_{\eta}$ of $Z$ is connected and $\left.s_{\alpha, \mathrm{dR}}\right|_{Z_{\eta}}$ is parallel, (2.3.6) sends $s_{\alpha, \mathrm{dR}, \tilde{x}}$ to $s_{\alpha, \mathrm{dR}, \tilde{x}^{\prime}}$ as required, and this proves the claim.

Since $Z$ and $R_{G_{W}}$ both have relative dimension $\mathrm{rk}_{W} \operatorname{gr}^{-1} \operatorname{Lie} G_{W}$ over $W$, this shows that $Z \stackrel{\sim}{\longrightarrow} \operatorname{Spf} R_{G_{W}}$. As $\tilde{x}$ was an arbitrary point of $\mathscr{S}_{K}^{-}(G, X)$ lifting $x$, this proves the proposition.

(2.3.7) We are now ready to prove the first main theorem of this paper. To explain it we recall the extension property of integral canonical models. This was formulated by Milne [Mi 1, §2], in order to give a characterization of the integral models for non-proper Shimura varieties whose existence had been conjectured by Langlands. We follow, in part, the treatment in [Mo, §3].

A scheme or pro-scheme $X$ over $\mathcal{O}_{(v)}$ is said to have the extension property if for any regular, formally smooth $\mathcal{O}_{(v)}$-scheme $S$, a map $S \otimes E \rightarrow X$ extends to $S$.

For each $K^{p} \subset G\left(\mathbb{A}_{f}^{p}\right)$ sufficiently small, we now fix $K^{\prime p} \subset \operatorname{GSp}\left(\mathbb{A}_{f}^{p}\right)$ so that there is an embedding

$$
\operatorname{Sh}_{K}(G, X) \hookrightarrow \operatorname{Sh}_{K^{\prime}}\left(\mathrm{GSp}, S^{ \pm}\right) .
$$

If $p=2$, then we continue to assume that the condition (2.3.4) holds.

Theorem (2.3.8). For $K=K_{p} K^{p}$, let $\mathscr{S}_{K}(G, X)$ denote the normalization of $\mathscr{S}_{K}^{-}(G, X)$, and set

$$
\mathscr{S}_{K_{p}}(G, X)=\lim _{K^{p}} \mathscr{S}_{K_{p} K^{p}}(G, X),
$$


where $K^{p} \subset G\left(\mathbb{A}_{f}^{p}\right)$ runs over sufficiently small compact open subgroups of $G\left(\mathbb{A}_{f}^{p}\right)$. Then

(1) $\mathscr{S}_{K_{p}}(G, X)$ is an inverse limit of smooth $\mathcal{O}_{(v)}$-schemes with finite étale transition maps, whose restriction to $E$ may be $G\left(\mathbb{A}_{f}^{p}\right)$-equivariantly identified with $\operatorname{Sh}_{K_{p}}(G, X)$.

(2) $\mathscr{S}_{K_{p}}(G, X)$ has the extension property, and in particular depends only on $(G, X)$ and $K_{p}$, and not on the symplectic embedding $i$.

Proof. The first claim follows directly from (2.3.5). For the second, suppose that $S$ is regular and formally smooth over $\mathcal{O}_{(v)}$. A morphism $S \otimes E \rightarrow \mathscr{S}_{K_{p}^{\prime}}\left(\mathrm{GSp}, S^{ \pm}\right)$can be extended to the height 1 primes by the argument of [Mi 1, Prop. 2.13] and then to all of $S$ by a result of Faltings $[\mathrm{Mo}, 3.6] 13$ Hence a morphism $S \otimes E \rightarrow \mathrm{Sh}_{K_{p}}(G, X)$ extends to a map $S \rightarrow \mathscr{S}_{K_{p}}^{-}(G, X)$ and this map lifts to $\mathscr{S}_{K_{p}}(G, X)$ since $S$ is formally smooth. This proves (2).

Corollary (2.3.9). Let $\mathcal{V}^{\circ}$ be the vector bundle on $\mathscr{S}_{K_{p}}(G, X)$ obtained by pulling back the de Rham cohomology of the universal abelian scheme over $\mathscr{S}_{K_{p}^{\prime}}\left(\mathrm{GSp}, S^{ \pm}\right)$. Then the sections $s_{\alpha, \mathrm{dR}} \in \mathcal{V}^{\otimes}$ extend to $G\left(\mathbb{A}_{f}^{p}\right)$-invariant sections of $\mathcal{V}^{\circ \otimes}$ over $\mathcal{O}_{(v)}$.

Proof. It suffices to show that for $K^{p}$ sufficiently small, and any closed point $x \in$ $\mathscr{S}_{K}(G, X)$ of characteristic $p$, the $s_{\alpha, \mathrm{dR}}$ extend to sections of $\mathcal{V}^{\circ, \otimes}$ over the formal neighbourhood of $x, \widehat{N}_{x} \subset \mathscr{S}_{K}(G, X)$.

To see this we use the notation of the proof of (2.3.5), which shows that $\widehat{N}_{x}$ may be identified with $\operatorname{Spf} R_{G_{W}}$. As in $\S 1.5$, let $\mathscr{G}_{R_{G_{W}}}$ denote the tautological deformation of $\mathscr{G}$ to $R_{G_{W}}$. By the construction in (1.5.4), the tensors $s_{\alpha, 0} \in \mathbb{D}\left(\mathscr{G}_{0}\right)(W)^{\otimes}$ lift to parallel tensors $s_{\alpha, \mathrm{dR}}^{\prime}$ in $\mathbb{D}\left(\mathscr{G}_{R_{G_{W}}}\right)\left(R_{G_{W}}\right)^{\otimes}$. The proof of (2.3.5) shows that $s_{\alpha, \mathrm{dR}}$ and $s_{\alpha, \mathrm{dR}}^{\prime}$ have the same specialization at a Zariski dense set of closed points of Spec $R_{G_{W}}[1 / p]$. Hence $s_{\alpha, \mathrm{dR}}=s_{\alpha, \mathrm{dR}}^{\prime}$.

\section{Integral CANONICAL MOdEls of ABELIAN TyPe}

(3.1) Twisting abelian varieties. In this subsection we deduce the existence of integral canonical models for Shimura varieties of abelian type from the results of $\S 2$.

We begin with a construction which twists an abelian variety by a torsor. Let $Z$ be an affine group scheme of finite type over $\mathbb{Q}$. We will write $\mathcal{O}_{Z}$ for the Hopf algebra of $Z$. Let $\mathcal{P}$ be a $Z$-torsor, and $\mathcal{O}_{\mathcal{P}}$ the ring of functions on $\mathcal{P}$.

Lemma (3.1.1). Let $V$ be a $\mathbb{Q}$-vector space equipped with the structure of an $\mathcal{O}_{Z}$ comodule. Then

(1) $V$ is a union of finite dimensional $\mathcal{O}_{Z}$-comodules.

(2) The natural map

$$
\left(V \otimes_{\mathbb{Q}} \mathcal{O}_{\mathcal{P}}\right)^{Z} \otimes_{\mathbb{Q}} \mathcal{O}_{\mathcal{P}} \stackrel{\sim}{\longrightarrow} V \otimes_{\mathbb{Q}} \mathcal{O}_{\mathcal{P}}
$$

is an isomorphism.

Proof. The first claim is in Wa, 3.3]. This shows that it is enough to check (2) for finite dimensional comodules, which is well known.

\footnotetext{
${ }^{13}$ This is stated in $\mathrm{Mo}$ for $p>2$, but when the second condition in (2.3.4) holds the same proof works for $p=2$.
} 
(3.1.2) Let $S$ be a scheme and $\mathcal{A} / S$ an abelian $S$-scheme. We will consider $\mathcal{A}$ in the category of abelian schemes up to isogeny, which is obtained from the category of abelian $S$-schemes by tensoring the Hom groups by $\otimes \mathbb{Q}$. An object in this category will be called an abelian scheme up to isogeny. An isomorphism in this category will be called a quasi-isogeny. For $T$ an $S$-scheme we set $\mathcal{A}(T)=\operatorname{Hom}_{S}(T, \mathcal{A}) \otimes_{\mathbb{Z}} \mathbb{Q}$.

Denote by $\underline{\operatorname{Aut}}_{\mathbb{Q}}(\mathcal{A})$ the $\mathbb{Q}$-group whose points in a $\mathbb{Q}$-algebra $R$ are given by

$$
\underline{\operatorname{Aut}}_{\mathbb{Q}}(\mathcal{A})(R)=\left(\left(\operatorname{End}_{S} \mathcal{A}\right) \otimes_{\mathbb{Z}} R\right)^{\times} .
$$

Let $Z$ and $\mathcal{P}$ be as above, and suppose that we are given a map of $\mathbb{Q}$-groups $Z \rightarrow$ Aut $_{\mathbb{Q}}(\mathcal{A})$. We define a pre-sheaf $\mathcal{A}^{\mathcal{P}}$ in the fppf topology of $S$ by setting

$$
\mathcal{A}^{\mathcal{P}}(T)=\left(\mathcal{A}(T) \otimes_{\mathbb{Q}} \mathcal{O}_{\mathcal{P}}\right)^{Z} .
$$

Lemma (3.1.3). $\mathcal{A}^{\mathcal{P}}$ is a sheaf, represented by an abelian scheme up to isogeny.

Proof. Using (3.1.1) one sees that $V \mapsto\left(V \otimes_{\mathbb{Q}} \mathcal{O}_{\mathcal{P}}\right)^{Z}$ is an exact functor, which implies that $\mathcal{A}^{\mathcal{P}}$ is a sheaf.

Let $E / \mathbb{Q}$ be a finite Galois extension such that $\mathcal{P}$ admits an $E$-valued point $\tilde{\gamma}$. Specializing the map in (3.1.1) by $\tilde{\gamma}$ yields an isomorphism of abelian sheaves

$$
\mathcal{A}^{\mathcal{P}} \otimes_{\mathbb{Q}} E \stackrel{\sim}{\longrightarrow} \mathcal{A} \otimes_{\mathbb{Q}} E .
$$

Now $\operatorname{Gal}(E / \mathbb{Q})$ acts on the left-hand side via its action on $E$. Transferring this action to the right-hand side of (3.1.3), we see that $\mathcal{A}^{\mathcal{P}}=\left(\mathcal{A} \otimes_{\mathbb{Q}} E\right)^{\mathrm{Gal}(E / \mathbb{Q})}$ so that $\mathcal{A}^{\mathcal{P}}$ is the kernel of a map $\alpha$ of abelian schemes up to isogeny. Such a kernel is represented by an abelian scheme up to isogeny, which can be constructed by realizing $\alpha$ as a map of abelian schemes and taking the connected component of the identity of the kernel.

(3.1.4) Keeping the above assumptions, denote by $\mathcal{A}^{*}$ the dual abelian scheme. There is a natural isomorphism $\alpha:$ End ${ }_{S} \mathcal{A} \stackrel{\sim}{\longrightarrow}\left(\text { End }_{S} \mathcal{A}^{*}\right)^{\text {op }}$ and hence an isomorphism

$$
\underline{\operatorname{Aut}}_{\mathbb{Q}}(\mathcal{A}) \stackrel{\sim}{\longrightarrow} \underline{\operatorname{Aut}}_{\mathbb{Q}}\left(\mathcal{A}^{*}\right) ; \quad g \mapsto \alpha(g)^{-1} .
$$

In particular we have a map $Z \rightarrow \underline{\operatorname{Aut}}_{\mathbb{Q}}\left(\mathcal{A}^{*}\right)$.

Let $c: Z \rightarrow \mathbb{G}_{m}$ be a character. We will denote by $\mathcal{A}(c)$ the abelian scheme $\mathcal{A}$ equipped with the map $Z \rightarrow{\underline{\text { uut }_{\mathbb{Q}}}}_{\mathcal{A}}$ obtained by multiplying the map in (3.1.2) by $c$.

Let $\lambda: \mathcal{A} \rightarrow \mathcal{A}^{*}$ be a weak polarization [De 1, 4.4]. We say that $\lambda$ is a $c$ polarization if the induced map $\mathcal{A} \rightarrow \mathcal{A}^{*}(c)$ is compatible with $Z$-actions.

Lemma (3.1.5). There is a natural isomorphism $\left(\mathcal{A}^{*}\right)^{\mathcal{P}} \stackrel{\sim}{\longrightarrow} \mathcal{A}^{\mathcal{P} *}$. If $\lambda: \mathcal{A} \rightarrow \mathcal{A}^{*}$ is a c-polarization, then there is a unique weak polarization $\lambda^{\mathcal{P}}: \mathcal{A}^{\mathcal{P}} \rightarrow \mathcal{A}^{\mathcal{P} *}$ such that the diagram

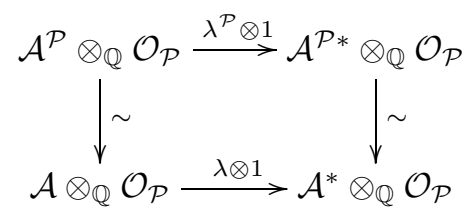

commutes up to an element of $\mathcal{O}_{\mathcal{P}}^{\times}$. Here the map on the right is obtained by composing $\mathcal{A}^{\mathcal{P} *} \stackrel{\sim}{\longrightarrow}\left(\mathcal{A}^{*}\right)^{\mathcal{P}}$ with the isomorphism of (3.1.1)(2). 
Proof. Let $\operatorname{Ext}^{1}\left(\mathcal{A}, \mathbb{G}_{m}\right)$ denote the sheaf in the fppf topology of $S$ whose value on an $S$-scheme $T$ is the group of extensions of $\mathcal{A}$ by $\mathbb{G}_{m}$, viewed as fppf sheaves on Spec $T$. Then there is a canonical isomorphism $\mathcal{A}^{*} \stackrel{\sim}{\longrightarrow} \underline{\operatorname{Ext}}^{1}\left(\mathcal{A}, \mathbb{G}_{m}\right)$ GRR, VIII $\S 3]$.

Since the functor $V \mapsto\left(V \otimes \mathcal{O}_{\mathcal{P}}\right)^{Z}$ of (3.1.1) is exact, we have

$$
\begin{aligned}
\underline{\operatorname{Ext}}^{1}\left(\mathcal{A}, \mathbb{G}_{m}\right) \otimes \mathcal{O}_{\mathcal{P}} & \stackrel{\sim}{\longrightarrow} \underline{\operatorname{Ext}}_{\mathcal{O}_{\mathcal{P}}}^{1}\left(\mathcal{A} \otimes \mathcal{O}_{\mathcal{P}}, \mathbb{G}_{m} \otimes \mathcal{O}_{\mathcal{P}}\right) \\
& \stackrel{\sim}{\longrightarrow} \underline{\operatorname{Ext}}_{\mathcal{O}_{\mathcal{P}}}^{1}\left(\mathcal{A}^{\mathcal{P}} \otimes \mathcal{O}_{\mathcal{P}}, \mathbb{G}_{m} \otimes \mathcal{O}_{\mathcal{P}}\right) \stackrel{\sim}{\longrightarrow} \underline{\operatorname{Ext}}^{1}\left(\mathcal{A}^{\mathcal{P}}, \mathbb{G}_{m}\right) \otimes \mathcal{O}_{\mathcal{P}} .
\end{aligned}
$$

Taking $Z$-invariants on both sides yields an isomorphism

$$
\left(\mathcal{A}^{*}\right)^{\mathcal{P}} \stackrel{\sim}{\longrightarrow}\left(\underline{\operatorname{Ext}}^{1}\left(\mathcal{A}, \mathbb{G}_{m}\right) \otimes \mathcal{O}_{\mathcal{P}}\right)^{Z} \stackrel{\sim}{\longrightarrow} \underline{\operatorname{Ext}}^{1}\left(\mathcal{A}^{\mathcal{P}}, \mathbb{G}_{m}\right) \otimes \mathcal{O}_{\mathcal{P}}^{Z} \stackrel{\sim}{\longrightarrow} \mathcal{A}^{\mathcal{P} *}
$$

Now let $\lambda: \mathcal{A} \rightarrow \mathcal{A}^{*}$ be a $c$-polarization, and let $f_{c} \in \mathcal{O}_{\mathcal{P}}^{\times}$be a function such that $Z$ acts on $f_{c}$ via $c$. Let $\tilde{\lambda}^{\mathcal{P}}: \mathcal{A}^{\mathcal{P}} \otimes_{\mathbb{Q}} \mathcal{O}_{\mathcal{P}} \rightarrow \mathcal{A}^{\mathcal{P} *}(c) \otimes_{\mathbb{Q}} \mathcal{O}_{\mathcal{P}}$ be the isomorphism making the diagram

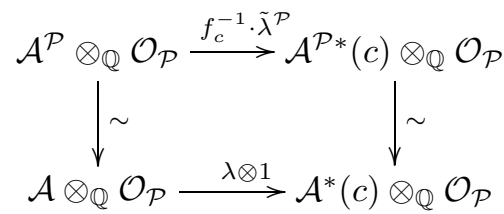

commute. Here the map on the right is obtained by twisting the composite

$$
\mathcal{A}^{\mathcal{P} *} \otimes_{\mathbb{Q}} \mathcal{O}_{\mathcal{P}} \stackrel{\sim}{\longrightarrow} \mathcal{A}^{* \mathcal{P}} \otimes_{\mathbb{Q}} \mathcal{O}_{\mathcal{P}} \stackrel{\sim}{\longrightarrow} \mathcal{A}^{*} \otimes_{\mathbb{Q}} \mathcal{O}_{\mathcal{P}}
$$

by $c$. By definition, the bottom map is $Z$-equivariant, as are the restrictions of the vertical maps to $\mathcal{A}^{\mathcal{P}}$ and $\mathcal{A}^{\mathcal{P} *}$, respectively. (Here we regard $\mathcal{A}^{\mathcal{P}}$ and $\mathcal{A}^{\mathcal{P} *}$ as equipped with the trivial $Z$-action, so $Z$ acts on $\mathcal{A}^{\mathcal{P} *}(c)$ via $c$.) Hence $\tilde{\lambda}^{\mathcal{P}}$ sends $\mathcal{A}^{\mathcal{P}}$ to $\mathcal{A}^{\mathcal{P} *}(c)$ and induces an isomorphism $\lambda^{\mathcal{P}}: \mathcal{A}^{\mathcal{P}} \stackrel{\sim}{\longrightarrow} \mathcal{A}^{\mathcal{P} *}(c)$.

Finally to see that $\lambda^{\mathcal{P}}: \mathcal{A}^{\mathcal{P}} \stackrel{\sim}{\longrightarrow} \mathcal{A}^{\mathcal{P} *}$ is a weak polarization, choose a finite extension $F / \mathbb{Q}$ such that $\mathcal{P}(F)$ is non-empty, and specialize (3.1.6) by some $F$ valued point. One checks easily that $\lambda^{\mathcal{P}}$ is a weak polarization if and only if the induced map

$$
\mathcal{A}^{\mathcal{P}} \otimes_{\mathbb{Q}} F \stackrel{\sim}{\longrightarrow} \mathcal{A}^{\mathcal{P} *} \otimes_{\mathbb{Q}} F
$$

is. The latter map is a weak polarization as $\lambda$ is.

(3.2) The action of $G^{\text {ad }}(\mathbb{Q})$. Let $(G, X)$ be a Shimura datum. Denote by $G^{\text {ad }}(\mathbb{R})_{1}$ the image of $G(\mathbb{R})$ in $G^{\text {ad }}(\mathbb{R})$, and set $G^{\text {ad }}(\mathbb{Q})_{1}=G^{\text {ad }}(\mathbb{Q}) \cap G^{\text {ad }}(\mathbb{R})_{1}$. Recall [De 3, §2] that there is an action of $G^{\text {ad }}(\mathbb{Q})_{1}$ on

$$
\operatorname{Sh}(G, X)=\lim _{K} \operatorname{Sh}_{K}(G, X)
$$

defined as follows. On complex points, $\gamma \in G^{\text {ad }}(\mathbb{Q})_{1}$ takes

$$
(h, g) \in G(\mathbb{Q}) \backslash X \times G\left(\mathbb{A}_{f}\right)
$$

to $\left(\gamma(h), \gamma g \gamma^{-1}\right)$, and this action is defined over $E=E(G, X)$ [De 3, 2.7.12].

Our first task is to give another description of this action in the case when $(G, X)$ is of Hodge type. Suppose that there is an embedding $(G, X) \hookrightarrow\left(\mathrm{GSp}, S^{ \pm}\right)$, where GSp is defined by a symplectic $\mathbb{Q}$-vector space $V=V_{\mathbb{Q}}$, as in $\S 2$. We fix compact open subgroups $K \subset G\left(\mathbb{A}_{f}\right)$ and $K^{\prime} \subset \operatorname{GSp}\left(\mathbb{A}_{f}\right)$ such that there is an induced embedding of $E$-schemes:

$$
\mathrm{Sh}_{K}(G, X) \hookrightarrow \mathrm{Sh}_{K^{\prime}}\left(\mathrm{GSp}, S^{ \pm}\right) .
$$


Let $\mathcal{A}$ denote the abelian scheme over the $E$-scheme $\operatorname{Sh}_{K}(G, X)$, obtained by pulling back the universal abelian scheme on $\operatorname{Sh}_{K^{\prime}}\left(\mathrm{GSp}, S^{ \pm}\right)$. If $x \in \operatorname{Sh}_{K}(G, X)(\mathbb{C})$, then a lifting of $x$ to $X \times G\left(\mathbb{A}_{f}\right)$ determines an isomorphism $V_{\mathbb{Q}} \stackrel{\sim}{\longrightarrow} H_{1}\left(\mathcal{A}_{x}(\mathbb{C}), \mathbb{Q}\right)$. For any two liftings these isomorphisms differ by an element of $G(\mathbb{Q})$ acting on $V_{\mathbb{Q}}$. Hence the composite

$$
Z=Z(G) \hookrightarrow G \hookrightarrow \mathrm{GL}\left(H_{1}\left(\mathcal{A}_{x}(\mathbb{C}), \mathbb{Q}\right)\right)
$$

depends only on $x$.

The following lemma is presumably well known.

Lemma (3.2.2). There exists an embedding $Z \hookrightarrow{\underline{\text { uut }_{\mathbb{Q}}}}_{\mathcal{A}}$ which induces the embedding (3.2.1) for any $x \in \operatorname{Sh}_{K}(G, X)(\mathbb{C})$.

Proof. We will write End $\mathcal{A}(\mathbb{C})$ for the endomorphisms of $\mathcal{A}(\mathbb{C})$ viewed as a family of complex tori over $\operatorname{Sh}_{K}(G, X)(\mathbb{C})$, and we set End $\mathbb{Q} \mathcal{A}(\mathbb{C})=$ End $\mathcal{A}(\mathbb{C}) \otimes_{\mathbb{Z}} \mathbb{Q}$.

Let $\underline{\text { uut }}_{\mathbb{Q}} \mathcal{A}(\mathbb{C})$ denote the $\mathbb{Q}$-group such that for a $\mathbb{Q}$-algebra $R$ we have

$$
\left(\underline{\operatorname{Aut}}_{\mathbb{Q}} \mathcal{A}(\mathbb{C})\right)(R)=\left(\operatorname{End}_{\mathbb{Q}} \mathcal{A}(\mathbb{C}) \otimes_{\mathbb{Q}} R\right)^{\times} .
$$

Then $\underline{A u t}_{\mathbb{Q}} \mathcal{A}$ is a closed subgroup of $\underline{\operatorname{Aut}}_{\mathbb{Q}} \mathcal{A}(\mathbb{C})$, and a point of $\underline{\operatorname{Aut}}_{\mathbb{Q}} \mathcal{A}(\mathbb{C})$ lies on Aut $_{\mathbb{A}} \mathcal{A}$ if and only if the corresponding ( $R$-valued) endomorphism of $\mathcal{A}(\mathbb{C})$ is induced by an endomorphism of the abelian scheme $\mathcal{A}$ over the $E$-scheme $\operatorname{Sh}_{K}(G, X)$.

For $x \in \mathrm{Sh}_{K}(G, X)(\mathbb{C})$, the image of the composite

$$
G \stackrel{(3.2 .1)}{\rightarrow} \operatorname{GL}\left(H^{1}\left(\mathcal{A}_{x}(\mathbb{C}), \mathbb{Q}\right)\right) \rightarrow \operatorname{End}_{\mathbb{Q}}\left(H^{1}\left(\mathcal{A}_{x}(\mathbb{C}), \mathbb{Q}\right)\right)
$$

depends only on $x$. Its commutant is a subalgebra of End $\mathbb{Q}(\mathbb{C})$. In particular we see that there is an inclusion $Z \subset \underline{\text { Aut }}_{\mathbb{Q}} \mathcal{A}(\mathbb{C})$ which induces the embedding (3.2.1) for any complex point $x$.

To see that this makes $Z$ a subgroup of Aut $_{\mathbb{Q}} \mathcal{A}$, note that under the action of $G\left(\mathbb{A}_{f}\right)$ on the tower $\lim _{K} \operatorname{Sh}_{K}(G, X), Z(\mathbb{Q})$ acts trivially. Hence we see that

$$
Z(\mathbb{Q}) \subset\left(\underline{\text { Aut }}_{\mathbb{Q}} \mathcal{A}\right)(\mathbb{Q}) .
$$

Let $Z^{0} \subset Z$ denote the connected component of the identity. As $Z^{0}(\mathbb{Q})$ is Zariski dense in $Z^{0}$ (for example using the real approximation theorem), we see that $Z^{0} \subset$ Aut $_{\mathbb{Q}} \mathcal{A}$.

Now suppose that $z \in Z(R)$ for some finite $\mathbb{Q}$-algebra $R$. Fix an embedding $\bar{E} \subset \mathbb{C}$. Since an $R$-valued endomorphism of $\mathcal{A}(\mathbb{C})$ is determined by its action on the $l$-adic Tate module $\lim _{n} \mathcal{A}\left[l^{n}\right]$, for any prime $l, z$ is induced by an automorphism of $\mathcal{A}$ over $\bar{E}$. We have to check that $\sigma^{*}(z)=z$ for every $\sigma \in \operatorname{Gal}(\bar{E} / E)$. Let $y \in \operatorname{Sh}_{K}(G, X)\left(E_{y}\right)$ be a special point defined over its reflex field $E_{y}$ and associated to a maximal torus $T \subset G$. The observation of the previous paragraph applied to $T$ implies that for $\tau \in \operatorname{Gal}\left(\bar{E} / E_{y}\right), \tau^{*}(z)=z$ over the connected component of $\operatorname{Sh}_{K}(G, X)$ containing $y$. Since $G\left(\mathbb{A}_{f}\right)$ acts transitively on the connected components of $\operatorname{Sh}(G, X)$, this implies that $\tau^{*}(z)=z$.

Finally, by [De 1, 5.1] and its proof, $y$ may be chosen so that $E_{y}$ is linearly disjoint from any finite extension of $E$. This implies that the subgroups $\operatorname{Gal}\left(\bar{E} / E_{y}\right)$ generate $\operatorname{Gal}(\bar{E} / E)$ (cf. [De 3, 2.6.3]).

(3.2.4) We will need a description of the points of $\operatorname{Sh}\left(\mathrm{GSp}, S^{ \pm}\right)$in terms of abelian varieties up to isogeny. This is slightly different from that sketched in 
$\S 2$ but is more convenient for describing the actions of $\operatorname{GSp}\left(\mathbb{A}_{f}\right)$ and $G^{\text {ad }}(\mathbb{Q})$ on $\operatorname{Sh}(G, X)$.

For an $E$-scheme $T$, and an abelian scheme $\mathcal{B}$ over $T$, we set

$$
\widehat{V}(\mathcal{B})=\lim _{n} \mathcal{B}[n]
$$

viewed as a local system on $T$. Let $\widehat{V}(\mathcal{B})_{\mathbb{Q}}=\widehat{V}(\mathcal{B}) \otimes_{\mathbb{Z}} \mathbb{Q}$. Note that $\mathcal{B} \mapsto \widehat{V}(\mathcal{B})_{\mathbb{Q}}$ is functorial for quasi-isogenies.

Recall [De 1, 4.20] that $\mathrm{Sh}_{K^{\prime}}\left(\mathrm{GSp}, S^{ \pm}\right)(T)$ is in canonical bijection with the set of isomorphism classes of triples $\left(\mathcal{A}_{x}, \lambda, \varepsilon_{K^{\prime}}\right)$, consisting of an abelian scheme up to isogeny $\mathcal{A}_{x}$ over $T$, equipped with a weak polarization $\lambda$, and a section

$$
\varepsilon_{K^{\prime}} \in \Gamma\left(T, \underline{\operatorname{Isom}}\left(V_{\mathbb{A}_{f}}, \widehat{V}\left(\mathcal{A}_{x}\right)_{\mathbb{Q}}\right) / K^{\prime}\right) .
$$

Here, as in (2.3.3), $\underline{\operatorname{Isom}}\left(V_{\mathbb{A}_{f}}, \widehat{V}\left(\mathcal{A}_{x}\right)_{\mathbb{Q}}\right)$ denotes the (pro)-étale sheaf of isomorphisms $V_{\mathbb{A}_{f}}=V \otimes \mathbb{Q} \mathbb{A}_{f} \stackrel{\sim}{\longrightarrow} \widehat{V}\left(\mathcal{A}_{x}\right)_{\mathbb{Q}}$ which is compatible with the pairings induced by $\psi$ and $\lambda$, up to a $\mathbb{A}_{f}^{\times}$-scalar.

Now suppose $x \in \operatorname{Sh}_{K}(G, X)(T)$. Then for any finite index subgroup $K_{1}^{\prime} \subset K^{\prime}$ containing $K$, there is an embedding

$$
\mathrm{Sh}_{K}(G, X) \hookrightarrow \mathrm{Sh}_{K_{1}^{\prime}}\left(\mathrm{GSp}, S^{ \pm}\right)
$$

so $x$ gives rise to a point of $\mathrm{Sh}_{K_{1}^{\prime}}\left(\mathrm{GSp}, S^{ \pm}\right)(T)$, and hence to a section

$$
\varepsilon_{K_{1}^{\prime}} \in \Gamma\left(T, \underline{\operatorname{Isom}}\left(V_{\mathbb{A}_{f}}, \widehat{V}\left(\mathcal{A}_{x}\right)_{\mathbb{Q}}\right) / K_{1}^{\prime}\right) .
$$

Passing to the limit over all such $K_{1}^{\prime}$, we obtain a section

$$
\varepsilon \in \Gamma\left(T, \underline{\operatorname{Isom}}\left(V_{\mathbb{A}_{f}}, \widehat{V}\left(\mathcal{A}_{x}\right)_{\mathbb{Q}}\right) / K\right) .
$$

Now let $\gamma \in G^{\text {ad }}(\mathbb{Q})$ and let $\mathcal{P} \subset G$ be the fibre of $\gamma$. By (3.2.2) we may apply the construction of (3.1) to $\mathcal{A}_{x}$ and obtain an abelian scheme (up to isogeny) $\mathcal{A}_{x}^{\mathcal{P}}$ over $T$. Moreover by (3.1.5), $\lambda$ induces a weak polarization $\lambda^{\mathcal{P}}: \mathcal{A}^{\mathcal{P}} \stackrel{\sim}{\longrightarrow} \mathcal{A}^{\mathcal{P} *}$.

Lemma (3.2.5). Let $F$ be a finite Galois extension of $\mathbb{Q}$ such that $\mathcal{P}(F)$ is nonempty, and let $\tilde{\gamma} \in \mathcal{P}(F)$. For $\sigma \in \operatorname{Gal}(F / \mathbb{Q})$ let $c_{\tilde{\gamma}}(\sigma)=\sigma(\tilde{\gamma}) \tilde{\gamma}^{-1} \in Z(F)$, and let

$$
\iota_{\tilde{\gamma}}: \mathcal{A}_{x}^{\mathcal{P}} \otimes_{\mathbb{Q}} F \stackrel{\sim}{\longrightarrow} \mathcal{A}_{x} \otimes_{\mathbb{Q}} F
$$

be the isomorphism obtained by specializing the map of (3.1.1)(2) at $\tilde{\gamma}$. Then

(1) $\sigma \circ \iota_{\tilde{\gamma}} \circ \sigma^{-1}=c_{\tilde{\gamma}}(\sigma)^{-1} \cdot \iota_{\tilde{\gamma}}$.

(2) The composite (defined étale locally on $T$ )

$$
\begin{gathered}
V \otimes_{\mathbb{Q}} \mathbb{A}_{f} \otimes_{\mathbb{Q}} F \stackrel{\tilde{\gamma}^{-1}}{\rightarrow} V \otimes_{\mathbb{Q}} \mathbb{A}_{f} \otimes_{\mathbb{Q}} F \stackrel{\varepsilon}{\rightarrow} \widehat{V}\left(\mathcal{A}_{x}\right)_{\mathbb{Q}} \otimes_{\mathbb{Q}} F \stackrel{i \tilde{\gamma}^{-1}}{\rightarrow} \widehat{V}\left(\mathcal{A}_{x}^{\mathcal{P}}\right)_{\mathbb{Q}} \otimes_{\mathbb{Q}} F \\
\text { is } \operatorname{Gal}(F / \mathbb{Q}) \text {-invariant and induces a section } \\
\varepsilon^{\mathcal{P}} \in \Gamma\left(T, \underline{\operatorname{Isom}}\left(V_{\mathbb{A}_{f}}, \widehat{V}\left(\mathcal{A}_{x}^{\mathcal{P}}\right)_{\mathbb{Q}}\right) / \gamma K \gamma^{-1}\right) .
\end{gathered}
$$

Proof. Consider the commutative diagram

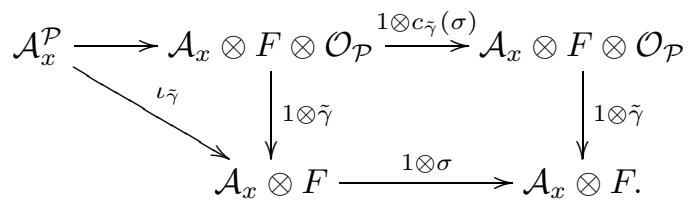


Since $\mathcal{A}_{x}^{\mathcal{P}}=\left(\mathcal{A}_{x} \otimes \mathcal{O}_{\mathcal{P}}\right)^{Z}$, we see that the composite of the maps in the top line is the composite

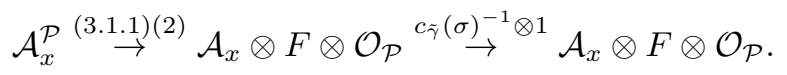

The formula in (1) now follows from the commutativity of the diagram. The claim in (2) follows from (1) by a simple calculation.

Lemma (3.2.6). Suppose that $\gamma \in G^{\mathrm{ad}}(\mathbb{Q})_{1}$, and let $K_{\gamma}^{\prime} \subset \mathrm{GSp}\left(\mathbb{A}_{f}\right)$ be a compact open subgroup containing $\gamma K \gamma^{-1}$ such that the induced map

$$
\operatorname{Sh}_{\gamma K \gamma^{-1}}(G, X) \rightarrow \operatorname{Sh}_{K_{\gamma}^{\prime}}\left(\mathrm{GSp}, S^{ \pm}\right)
$$

is a closed embedding. Then the map

$$
\operatorname{Sh}_{K}(G, X) \rightarrow \operatorname{Sh}_{K_{\gamma}^{\prime}}\left(\mathrm{GSp}, S^{ \pm}\right)
$$

given on T-valued points by the assignment

$$
\left(\mathcal{A}_{x}, \lambda, \varepsilon\right) \mapsto\left(\mathcal{A}_{x}^{\mathcal{P}}, \lambda^{\mathcal{P}}, \varepsilon^{\mathcal{P}}\right)
$$

factors through $\mathrm{Sh}_{\gamma K \gamma^{-1}}(G, X)$. The induced map

$$
\operatorname{Sh}_{K}(G, X) \rightarrow \operatorname{Sh}_{\gamma K \gamma^{-1}}(G, X)
$$

is the one attached to $\gamma$ in (3.2).

Proof. It suffices to check both statements on $\mathbb{C}$-valued points. Thus we may assume that $T=\operatorname{Spec} \mathbb{C}$. Recall that the point $(h, g) \in \mathrm{GSp}(\mathbb{Q}) \backslash S^{ \pm} \times \mathrm{GSp}\left(\mathbb{A}_{f}\right) / K$ attached to a triple $\left(\mathcal{A}_{x}, \lambda, \varepsilon\right)$ as above may be computed as follows: Choose an isomorphism $\alpha: H_{1}\left(\mathcal{A}_{x}(\mathbb{C}), \mathbb{Q}\right) \stackrel{\sim}{\longrightarrow} V_{\mathbb{Q}}$ respecting the polarizations induced by $\lambda$ and $\psi$ up to a $\mathbb{Q}^{\times}$-scalar. The Hodge decomposition then gives a grading of $V_{\mathbb{C}}$ which determines the cocharacter $h$, while the element $g$ is given by the composite

$$
V_{\mathbb{A}_{f}} \stackrel{\varepsilon}{\rightarrow} \widehat{V}\left(\mathcal{A}_{x}\right)_{\mathbb{Q}} \stackrel{\sim}{\longrightarrow} H_{1}\left(\mathcal{A}_{x}(\mathbb{C}), \mathbb{Q}\right) \otimes_{\mathbb{Q}} \mathbb{A}_{f} \stackrel{\alpha \otimes 1}{\rightarrow} V_{\mathbb{A}_{f}} .
$$

The resulting element of $\mathrm{GSp}(\mathbb{Q}) \backslash S^{ \pm} \times \operatorname{GSp}\left(\mathbb{A}_{f}\right) / K$ does not depend on the choice of $\alpha$.

Now let $F / \mathbb{Q}$ be a finite Galois extension and $\tilde{\gamma} \in \mathcal{P}(F)$. The same argument as in $(3.2 .5)$ shows that there exists an isomorphism $\alpha^{\mathcal{P}}: H_{1}\left(\mathcal{A}_{x}^{\mathcal{P}}(\mathbb{C}), \mathbb{Q}\right) \stackrel{\sim}{\longrightarrow} V_{\mathbb{Q}}$ such that the diagram

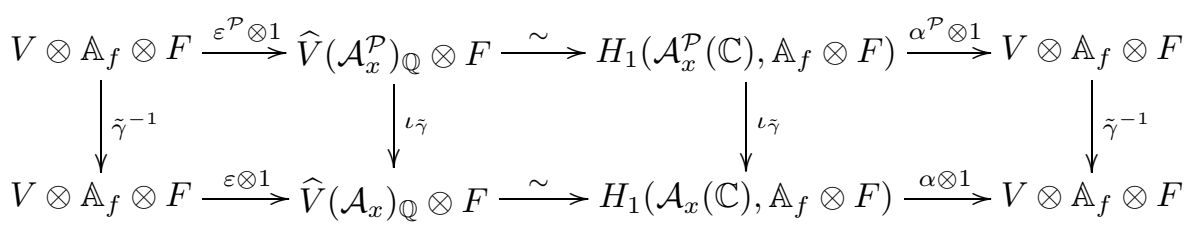

commutes. By (3.1.5), $\tilde{\gamma}^{-1} \circ\left(\alpha^{\mathcal{P}} \otimes 1\right)$ is compatible with polarizations up to an $F^{\times}$-scalar, so $\alpha^{\mathcal{P}}$ is compatible with polarizations up to a $\mathbb{Q}^{\times}$-scalar. Hence the maps obtained by taking $\operatorname{Gal}(F / \mathbb{Q})$-invariants in the top line may be used to compute the point of $\operatorname{GSp}(\mathbb{Q}) \backslash S^{ \pm} \times \operatorname{GSp}\left(\mathbb{A}_{f}\right) / K_{\gamma}^{\prime}$ corresponding to $\left(\mathcal{A}_{x}^{\mathcal{P}}, \lambda^{\mathcal{P}}, \varepsilon^{\mathcal{P}}\right)$. The commutativity of the diagram shows that this point is $\gamma(x)$. 
(3.3) Connected Shimura varieties. We now recall the relationship between Shimura varieties and connected Shimura varieties. This is a variant of [De 3, §2], which was already sketched in [Mo, §3].

(3.3.1) We recall the notation of [De 3, 2.0]. Let $H$ be a group equipped with an action of a group $\Delta$, and $\Gamma \subset H$ a $\Delta$-stable subgroup. Suppose given a $\Delta$ equivariant map $\varphi: \Gamma \rightarrow \Delta$, where $\Delta$ acts on itself by inner automorphisms, and suppose that for $\gamma \in \Gamma, \varphi(\gamma)$ acts on $H$ as inner conjugation by $\gamma$. Then the elements of the form $\left(\gamma, \varphi(\gamma)^{-1}\right)$ form a normal subgroup of the semi-direct product $H \rtimes \Delta$. We denote by $H *_{\Gamma} \Delta$ the quotient of $H \rtimes \Delta$ by this subgroup.

Converting the left action of $G^{\text {ad }}(\mathbb{Q})_{1}$ on $\operatorname{Sh}(G, X)$ into a right action, one obtains a right action of $G\left(\mathbb{A}_{f}\right) / Z(\mathbb{Q})^{-} *_{G(\mathbb{Q}) / Z(\mathbb{Q})} G^{\text {ad }}(\mathbb{Q})_{1}$ on $\operatorname{Sh}(G, X)$, where $Z(\mathbb{Q})^{-}$ denotes the closure of $Z(\mathbb{Q})$ in $G\left(\mathbb{A}_{f}\right)$.

For a subgroup $H \subset G(\mathbb{R})$, denote by $H_{+}$the preimage in $H$ of $G^{\text {ad }}(\mathbb{R})^{+}$, the connected component of the identity in $G^{\text {ad }}(\mathbb{R})$. As usual, we write $G^{\text {ad }}(\mathbb{Q})^{+}=$ $G^{\text {ad }}(\mathbb{Q}) \cap G^{\text {ad }}(\mathbb{R})^{+}$. Since $G(\mathbb{Q})$ is dense in $G(\mathbb{R})$ we have

$$
G\left(\mathbb{A}_{f}\right) / Z(\mathbb{Q})^{-} *_{G(\mathbb{Q}) / Z(\mathbb{Q})} G^{\mathrm{ad}}(\mathbb{Q})_{1} \stackrel{\sim}{\longrightarrow} G\left(\mathbb{A}_{f}\right) / Z(\mathbb{Q})^{-} *_{G(\mathbb{Q})_{+} / Z(\mathbb{Q})} G^{\mathrm{ad}}(\mathbb{Q})^{+} .
$$

We denote this group by $\mathscr{A}(G)$.

Let $G(\mathbb{Q})_{+}^{-}$denote the closure of $G(\mathbb{Q})_{+}$in $G\left(\mathbb{A}_{f}\right)$ and set

$$
\mathscr{A}(G)^{\circ}=G(\mathbb{Q})_{+}^{-} / Z(\mathbb{Q})^{-} *_{G(\mathbb{Q})_{+} / Z(\mathbb{Q})} G^{\mathrm{ad}}(\mathbb{Q})^{+} .
$$

This group depends only on $G^{\text {der }}$ and not on $G$; it is equal to the completion of $G^{\text {ad }}(\mathbb{Q})^{+}$with respect to the topology whose open sets are images of congruence subgroups in $G^{\text {der }}(\mathbb{Q})$ De 3, 2.1.15].

(3.3.2) Now let $G_{\mathbb{Z}_{(p)}}$ be a reductive group over $\mathbb{Z}_{(p)}$ with generic fibre $G$. To avoid overloading notation we will usually write $G\left(\mathbb{Z}_{(p)}\right)$ and $G\left(\mathbb{Z}_{p}\right)$ for $G_{\mathbb{Z}_{(p)}}\left(\mathbb{Z}_{(p)}\right)$ and $G_{\mathbb{Z}_{(p)}}\left(\mathbb{Z}_{p}\right)$.

Let $Z_{\mathbb{Z}_{(p)}}$ be the center of $G_{\mathbb{Z}_{(p)}}$ and $G_{\mathbb{Z}_{(p)}}^{\text {ad }}=G_{\mathbb{Z}_{(p)}} / Z_{\mathbb{Z}_{(p)}}$. Write $G\left(\mathbb{Z}_{(p)}\right)_{+}=$ $G\left(\mathbb{Z}_{(p)}\right) \cap G(\mathbb{Q})_{+}$and $G^{\text {ad }}\left(\mathbb{Z}_{(p)}\right)^{+}=G^{\text {ad }}\left(\mathbb{Z}_{(p)}\right) \cap G^{\text {ad }}(\mathbb{Q})^{+}$. We denote by $Z\left(\mathbb{Z}_{(p)}\right)^{-}$ the closure of $Z\left(\mathbb{Z}_{(p)}\right)$ in $Z\left(\mathbb{A}_{f}^{p}\right)$, and by $G\left(\mathbb{Z}_{(p)}\right)_{+}^{-}$the closure of $G\left(\mathbb{Z}_{(p)}\right)_{+}$in $G\left(\mathbb{A}_{f}^{p}\right)$. Set

and

$$
\mathscr{A}\left(G_{\mathbb{Z}_{(p)}}\right)=G\left(\mathbb{A}_{f}^{p}\right) / Z\left(\mathbb{Z}_{(p)}\right)^{-} *_{G\left(\mathbb{Z}_{(p)}\right)+/ Z\left(\mathbb{Z}_{(p)}\right)} G^{\text {ad }}\left(\mathbb{Z}_{(p)}\right)^{+}
$$

$$
\mathscr{A}\left(G_{\mathbb{Z}_{(p)}}\right)^{\circ}=G\left(\mathbb{Z}_{(p)}\right)_{+}^{-} / Z\left(\mathbb{Z}_{(p)}\right)^{-} *_{G\left(\mathbb{Z}_{(p)}\right)_{+} / Z\left(\mathbb{Z}_{(p)}\right)} G^{\mathrm{ad}}\left(\mathbb{Z}_{(p)}\right)^{+} .
$$

As in (3.3.1), using [De 3, 2.0.12], one sees that the latter group depends only on $G_{\mathbb{Z}_{(p)}}^{\text {der }}$ and not on $G_{\mathbb{Z}_{(p)}}$.

Now let $G_{2, \mathbb{Z}_{(p)}}$ be a reductive group over $\mathbb{Z}_{(p)}$, equipped with a central isogeny $\psi: G_{\mathbb{Z}_{(p)}}^{\text {der }} \rightarrow G_{2, \mathbb{Z}_{(p)}}^{\text {der }}$. We denote by $Z_{2, \mathbb{Z}_{(p)}}$ the center of $G_{2, \mathbb{Z}_{(p)}}$ and by $G_{2}$ and $Z_{2}$ the generic fibres of $G_{2, \mathbb{Z}_{(p)}}$, and $Z_{2, \mathbb{Z}_{(p)}}$, respectively.

Lemma (3.3.3). The natural map

$$
\mathscr{A}\left(G_{\mathbb{Z}_{(p)}}\right)^{\circ} \backslash \mathscr{A}\left(G_{2, \mathbb{Z}_{(p)}}\right) \rightarrow \mathscr{A}(G)^{\circ} \backslash \mathscr{A}\left(G_{2}\right) / G_{2}\left(\mathbb{Z}_{p}\right),
$$

where $G_{2}\left(\mathbb{Z}_{p}\right)$ acts on $\mathscr{A}\left(G_{2}\right)$ via right multiplication on $G_{2}\left(\mathbb{Q}_{p}\right)$, is an isomorphism of abelian groups.

Proof. We remind the reader that $\mathscr{A}(G)^{\circ}$ acts on $\mathscr{A}\left(G_{2}\right)$ via

$$
\mathscr{A}(G)^{\circ} \stackrel{\sim}{\longrightarrow} \mathscr{A}\left(G^{\text {der }}\right)^{\circ} \rightarrow \mathscr{A}\left(G_{2}^{\text {der }}\right)^{\circ} \rightarrow \mathscr{A}\left(G_{2}\right)
$$


and similarly for $\mathscr{A}\left(G_{\mathbb{Z}_{(p)}}\right)^{\circ}$. Recall that $\mathscr{A}\left(G_{\mathbb{Z}_{(p)}}\right)^{\circ} \backslash \mathscr{A}\left(G_{2, \mathbb{Z}_{(p)}}\right)$ and $\mathscr{A}(G)^{\circ} \backslash \mathscr{A}\left(G_{2}\right)$ are abelian groups since, as in [De 3, 2.5.1], the strong approximation theorem implies that $G(\mathbb{Q})_{+}^{-}\left(\operatorname{resp} . G\left(\mathbb{Z}_{(p)}\right)_{+}^{-}\right)$contains the image of $\tilde{G}\left(\mathbb{A}_{f}\right)\left(\operatorname{resp} . \tilde{G}\left(\mathbb{A}_{f}^{p}\right)\right)$, where $\tilde{G}$ is the universal cover of $G^{\text {der }}$. This shows that the map in the lemma is a morphism of abelian groups.

To check that (3.3.4) is surjective let $(g, \gamma) \in \mathscr{A}\left(G_{2}\right)$ with $g \in G_{2}\left(\mathbb{A}_{f}\right)$ and $\gamma \in G_{2}^{\text {ad }}(\mathbb{Q})^{+}$. By $(2.2 .6)$, after multiplying $(g, \gamma)$ on the right by an element of $G_{2}\left(\mathbb{Z}_{p}\right)$, we may assume that $g_{p}$, the $p$-component of $g$, is trivial. Then multiplying on the left by an element of $\mathscr{A}(G)^{\circ}$, we may assume in addition that $\gamma=1$. Then $(g, 1)$ is clearly in the image of (3.3.4).

Next we check injectivity. Let $(g, \gamma) \in \mathscr{A}\left(G_{2, \mathbb{Z}_{(p)}}\right)$, where we view $g \in G_{2}\left(\mathbb{A}_{f}\right)$ as an element having trivial $p$-component. Multiplying by an element of $\mathscr{A}\left(G_{\mathbb{Z}_{(p)}}\right)^{\circ}$, we may assume $\gamma=1$. Suppose that $(g, 1)$ is in the kernel of (3.3.4). Then there exists $\left(g_{1}, \gamma_{1}\right) \in \mathscr{A}(G)^{\circ}$ and $h \in G_{2}\left(\mathbb{Z}_{p}\right)$ such that

$$
(g, 1)=\left(g_{1}, \gamma_{1}\right) \cdot h=\left(g_{1}, \gamma_{1}\right)(h, 1)=\left(g_{1} \gamma_{1} h \gamma_{1}^{-1}, \gamma_{1}\right)
$$

in $\mathscr{A}\left(G_{2}\right)$. Hence $g=g_{1} \gamma_{1} h$ in $G_{2}\left(\mathbb{A}_{f}\right) / Z_{2}(\mathbb{Q})^{-}$.

Since $g_{1}$ may be approximated by elements of $G(\mathbb{Q})_{+}$, we may replace $\left(g_{1}, \gamma_{1}\right)$ by a pair which represents the same element of $\mathscr{A}(G)^{\circ}$ and such that $g_{1 p} \in G_{2}\left(\mathbb{Z}_{p}\right)$. Since $\gamma_{1}=g_{1}^{-1} g h^{-1}$, comparing $p$-components then shows that $\gamma_{1} \in G^{\text {ad }}\left(\mathbb{Z}_{(p)}\right)^{+}$.

Hence we have $(g, 1)=\left(g_{1} \gamma_{1} h \gamma_{1}^{-1}, \gamma_{1}\right)$ in

$$
\left[G_{2}\left(\mathbb{A}_{f}^{p}\right) \times G_{2}\left(\mathbb{Z}_{p}\right)\right] / Z_{2}\left(\mathbb{Z}_{(p)}\right)^{-} *_{G_{2}\left(\mathbb{Z}_{(p)}\right)_{+} / Z_{2}\left(\mathbb{Z}_{(p)}\right)} G_{2}^{\mathrm{ad}}\left(\mathbb{Z}_{(p)}\right)^{+} \subset \mathscr{A}\left(G_{2}\right) .
$$

Dividing both sides by $G_{2}\left(\mathbb{Z}_{(p)}\right)$ shows that $\left(g^{p}, 1\right)=\left(g_{1}^{p}, \gamma_{1}\right)$ in $\mathscr{A}\left(G_{2, \mathbb{Z}_{(p)}}\right)$.

(3.3.5) As in [De 3, 2.7], for a locally compact, totally disconnected group $\Gamma$, a scheme with continuous $\Gamma$-action is an inverse system of quasi-projective14 schemes $S=\lim _{K} S_{K}$ indexed by the compact open subgroups $K \subset \Gamma$, and equipped with a right action of $\Gamma$, induced by isomorphisms $\gamma: S_{K} \stackrel{\sim}{\longrightarrow} S_{\gamma^{-1} K \gamma}$, which are the identity if $\gamma \in K$, and such that $S_{K} /\left(K^{\prime} / K\right) \stackrel{\sim}{\longrightarrow} S_{K^{\prime}}$ for $K \subset K^{\prime} \subset \Gamma$ compact open. In particular the maps in the inverse limit are finite, so $S$ may also be viewed as a scheme.

If $\Gamma \rightarrow \Gamma^{\prime}$ is a map of locally compact, totally disconnected groups, with compact kernel, then $\Gamma$ acts on the scheme $\Gamma^{\prime} \times S$ by $\left(\gamma^{\prime}, s\right) \gamma=\left(\gamma^{-1} \gamma^{\prime}, s \gamma\right)$. The quotient scheme $S^{\prime}=\Gamma^{\prime} \times S / \Gamma$ is associated to a scheme with continuous $\Gamma^{\prime}$-action, $\lim S_{K^{\prime}}^{\prime}$, where $S_{K^{\prime}}^{\prime}=S^{\prime} / K^{\prime}$. The quotient $S_{K^{\prime}}^{\prime}$ also has an explicit description in terms of the $S_{K}$.

(3.3.6) As in $\S 2$, we let $E=E(G, X)$, and we fix an algebraic closure $\bar{E}$ of $E$. Let $E^{p} \subset \bar{E}$ be the maximal extension of $E$ which is unramified at primes dividing $p$. We set $K_{p}=G\left(\mathbb{Z}_{p}\right)$ and $K_{2, p}=G_{2}\left(\mathbb{Z}_{p}\right)$, and we write $K=K_{p} K^{p}$ for a compact open subgroup of $G\left(\mathbb{A}_{f}\right)$.

We now assume we are given a Shimura datum $\left(G_{2}, X_{2}\right)$ such that the isomorphism $G^{\text {ad }} \stackrel{\sim}{\longrightarrow} G_{2}^{\text {ad }}$ induces $\left(G^{\text {ad }}, X^{\text {ad }}\right) \stackrel{\sim}{\longrightarrow}\left(G_{2}^{\text {ad }}, X_{2}^{\text {ad }}\right)$. Here, as usual, $X^{\text {ad }}$ denotes the $G^{\text {ad }}(\mathbb{R})$-conjugacy class which contains the image of $X$.

\footnotetext{
${ }^{14}$ Over some base, which will be clear in applications.
} 
Fix a connected component $X^{+} \subset X$. For any compact open subgroup $K^{\prime} \subset$ $G\left(\mathbb{A}_{f}\right)$ we denote by $\operatorname{Sh}_{K^{\prime}}(G, X)^{+} \subset \operatorname{Sh}_{K^{\prime}}(G, X)$ the geometrically connected component which is the image of $X^{+} \times 1$. By (2.2.4), the geometrically connected components of $\operatorname{Sh}_{K}(G, X)$ are defined over $E^{p}$. In particular $\operatorname{Sh}_{K}(G, X)^{+}$is defined over $E^{p}$. We write

$$
\operatorname{Sh}_{K_{p}}(G, X)^{+}:=\lim _{K^{p}} \operatorname{Sh}_{K}(G, X)^{+}
$$

and $\operatorname{Sh}(G, X)^{+}$for the limit over all compact open subgroups of $G\left(\mathbb{A}_{f}\right)$.

Lemma (3.3.7). Let $\mathscr{E}\left(G_{\mathbb{Z}_{(p)}}^{\text {der }}\right) \subset \mathscr{A}\left(G_{\mathbb{Z}_{(p)}}\right) \times \operatorname{Gal}\left(E^{p} / E\right)$ denote the stabilizer of $\operatorname{Sh}_{K_{p}}(G, X)^{+} \subset \operatorname{Sh}_{K_{p}}(G, X)$ (viewed as $E^{p}$-schemes). Then

(1) $\mathscr{E}\left(G_{\mathbb{Z}_{(p)}}^{\text {der }}\right)$ is an extension of $\operatorname{Gal}\left(E^{p} / E\right)$ by $\mathscr{A}\left(G_{\mathbb{Z}_{(p)}}\right)^{\circ}$ which depends (as an extension) only on $G_{\mathbb{Z}_{(p)}}^{\mathrm{der}}$ and $X^{\mathrm{ad}}$, and not on $G_{\mathbb{Z}_{(p)}}$.

(2) There is a canonical isomorphism

$$
\mathscr{A}\left(G_{\mathbb{Z}_{(p)}}\right) * \mathscr{A}\left(G_{\mathbb{Z}_{(p)}}\right) \mathscr{E}\left(G_{\mathbb{Z}_{(p)}}^{\text {der }}\right) \stackrel{\sim}{\longrightarrow} \mathscr{A}\left(G_{\mathbb{Z}_{(p)}}\right) \times \operatorname{Gal}\left(E^{p} / E\right),
$$

where an element of $\mathscr{E}\left(G_{\mathbb{Z}_{(p)}}^{\text {der }}\right)$ acts on $\mathscr{A}\left(G_{\mathbb{Z}_{(p)}}\right)$ via conjugation by its image in $\mathscr{A}\left(G_{\mathbb{Z}_{(p)}}\right)$.

Proof. Consider the exact sequence

$$
0 \rightarrow \mathscr{A}(G)^{\circ} \rightarrow \mathscr{A}(G) \rightarrow \mathscr{A}(G)^{\circ} \backslash \mathscr{A}(G) \rightarrow 0 .
$$

The conjugacy class $X$ determines a map $r: \operatorname{Gal}(\bar{E} / E) \rightarrow \mathscr{A}(G) / \mathscr{A}(G)^{\circ}$ De 3, 2.5.9], and pulling (3.3.8) back by this map produces an extension $\mathscr{E}\left(G^{\text {der }}\right)$ of $\operatorname{Gal}(\bar{E} / E)$ by $\mathscr{A}(G)^{\circ}$. Clearly $\mathscr{A}(G) * \mathscr{A}(G)^{\circ} \mathscr{E}\left(G^{\text {der }}\right) \stackrel{\sim}{\longrightarrow} \mathscr{A}(G) \times \operatorname{Gal}(\bar{E} / E)$, and this isomorphism identifies $\mathscr{E}\left(G^{\text {der }}\right)$ with the stabilizer of $\operatorname{Sh}(G, X)^{+}$in $\mathscr{A}(G) \times$ $\operatorname{Gal}(\bar{E} / E)[$ De 3, 2.6.3].

Now let

$$
\tilde{\mathscr{A}}\left(G_{\mathbb{Z}_{(p)}}\right)=\left[G\left(\mathbb{A}_{f}^{p}\right) \times G\left(\mathbb{Z}_{p}\right)\right] / Z\left(\mathbb{Z}_{(p)}\right)^{-} *_{G\left(\mathbb{Z}_{(p)}\right)_{+} / Z\left(\mathbb{Z}_{(p)}\right)} G^{\mathrm{ad}}\left(\mathbb{Z}_{(p)}\right)^{+} \subset \mathscr{A}(G)
$$

and

$$
\tilde{\mathscr{A}}\left(G_{\mathbb{Z}_{(p)}}\right)^{\circ}=G\left(\mathbb{Z}_{(p)}\right)_{+}^{\sim} / Z\left(\mathbb{Z}_{(p)}\right)^{-} *_{G\left(\mathbb{Z}_{(p)}\right)+/ Z\left(\mathbb{Z}_{(p)}\right)} G^{\mathrm{ad}}\left(\mathbb{Z}_{(p)}\right)^{+} \subset \mathscr{A}(G)^{\circ},
$$

where $G\left(\mathbb{Z}_{(p)}\right) \sim$ denotes the closure of $G\left(\mathbb{Z}_{(p)}\right)_{+}$in $G\left(\mathbb{A}_{f}^{p}\right) \times G\left(\mathbb{Z}_{p}\right)$.

By (3.3.3) we have a bijection

$$
\tilde{\mathscr{A}}\left(G_{\mathbb{Z}_{(p)}}\right)^{\circ} \backslash \tilde{\mathscr{A}}\left(G_{\mathbb{Z}_{(p)}}\right) \stackrel{\sim}{\longrightarrow} \mathscr{A}(G)^{\circ} \backslash \mathscr{A}(G) .
$$

Hence (3.3.8) is obtained by pushout from the sequence

$$
0 \rightarrow \tilde{\mathscr{A}}\left(G_{\mathbb{Z}_{(p)}}\right)^{\circ} \rightarrow \tilde{\mathscr{A}}\left(G_{\mathbb{Z}_{(p)}}\right) \rightarrow \tilde{\mathscr{A}}\left(G_{\mathbb{Z}_{(p)}}\right)^{\circ} \backslash \tilde{\mathscr{A}}\left(G_{\mathbb{Z}_{(p)}}\right) \rightarrow 0,
$$

and pulling the latter sequence back by $r$ gives rise to an extension $\tilde{\mathscr{E}}\left(G_{\mathbb{Z}_{(p)}}^{\text {der }}\right)$ of $\operatorname{Gal}(\bar{E} / E)$ by $\tilde{\mathscr{A}}\left(G_{\mathbb{Z}_{(p)}}\right)^{\circ}$, which may be identified with the stabilizer of $\operatorname{Sh}(G, X)^{+} \subset$ $\operatorname{Sh}(G, X)$ in $\tilde{\mathscr{A}}\left(G_{\mathbb{Z}_{(p)}}\right) \times \operatorname{Gal}(\bar{E} / E)$. The same argument as in [De 3, 2.5.6] shows that this extension depends only on $G_{\mathbb{Z}_{(p)}}^{\text {der }}$.

Now the isomorphism $\tilde{\mathscr{A}}\left(G_{\mathbb{Z}_{(p)}}\right) / G\left(\mathbb{Z}_{p}\right) \stackrel{\sim}{\longrightarrow} \mathscr{A}\left(G_{\mathbb{Z}_{(p)}}\right)$ induces an isomorphism

$$
\tilde{\mathscr{A}}\left(G_{\mathbb{Z}_{(p)}}\right)^{\circ} /\left(\tilde{\mathscr{A}}\left(G_{\mathbb{Z}_{(p)}}\right)^{\circ} \cap G\left(\mathbb{Z}_{p}\right)\right) \stackrel{\sim}{\longrightarrow} \mathscr{A}\left(G_{\mathbb{Z}_{(p)}}\right)^{\circ},
$$


so the quotient $\tilde{\mathscr{E}}\left(G_{\mathbb{Z}_{(p)}}^{\text {der }}\right) /\left(\tilde{\mathscr{A}}\left(G_{\mathbb{Z}_{(p)}}\right)^{\circ} \cap G\left(\mathbb{Z}_{p}\right)\right)$ is an extension of $\operatorname{Gal}(\bar{E} / E)$ by $\mathscr{A}\left(G_{\mathbb{Z}_{(p)}}\right)^{\circ}$ which may be identified with the stabilizer of

$$
\operatorname{Sh}(G, X)^{+} /\left[\mathscr{A}(G)^{\circ} \cap G\left(\mathbb{Z}_{p}\right)\right]=\operatorname{Sh}_{K_{p}}(G, X)^{+} \subset \operatorname{Sh}_{K_{p}}(G, X)=\operatorname{Sh}(G, X) / G\left(\mathbb{Z}_{p}\right)
$$

in $\mathscr{A}\left(G_{\mathbb{Z}_{(p)}}\right) \times \operatorname{Gal}(\bar{E} / E)$.

Finally, since $\operatorname{Sh}_{K_{p}}(G, X)^{+}$is defined over $E^{p}, \operatorname{Gal}\left(\bar{E} / E^{p}\right) \subset \tilde{\mathscr{E}}\left(G_{\mathbb{Z}_{(p)}}^{\text {der }}\right)$, and the quotient $\operatorname{Gal}\left(\bar{E} / E^{p}\right) \backslash \tilde{\mathscr{E}}\left(G_{\mathbb{Z}_{(p)}}^{\text {der }}\right) /\left(\tilde{\mathscr{A}}\left(G_{\mathbb{Z}_{(p)}}\right)^{\circ} \cap G\left(\mathbb{Z}_{p}\right)\right)$ is the required extension of $\operatorname{Gal}\left(E^{p} / E\right)$ by $\mathscr{A}\left(G_{\mathbb{Z}_{(p)}}\right)^{\circ}$.

(3.3.9) Consider the right action of $\mathscr{E}\left(G_{\mathbb{Z}_{(p)}}^{\text {der }}\right)$ on $\mathscr{A}\left(G_{2, \mathbb{Z}_{(p)}}\right) \times \operatorname{Sh}_{K_{p}}(G, X)^{+}$ given by $(a, s) e=\left(\bar{e}^{-1} a \bar{e}, s e\right)$, where $\bar{e}$ denotes the image of $e$ in $\mathscr{A}\left(G_{2, \mathbb{Z}_{(p)}}\right)$. This extends uniquely to an action of the semi-direct product $\mathscr{A}\left(G_{2, \mathbb{Z}_{(p)}}\right) \rtimes \mathscr{E}\left(G_{\mathbb{Z}_{(p)}}^{\text {der }}\right)$ in which $\mathscr{A}\left(G_{2, \mathbb{Z}_{(p)}}\right)$ acts by $(a, s) \cdot a^{\prime}=\left(a a^{\prime}, s\right)$. This action descends to an action of $\mathscr{A}\left(G_{2, \mathbb{Z}_{(p)}}\right) * \mathscr{A}\left(G_{\mathbb{Z}_{(p)}}\right) \circ \mathscr{E}\left(G_{\mathbb{Z}_{(p)}}^{\text {der }}\right)$ on $\left[\mathscr{A}\left(G_{2, \mathbb{Z}_{(p)}}\right) \times \operatorname{Sh}_{K_{p}}(G, X)^{+}\right] / \mathscr{A}\left(G_{\mathbb{Z}_{(p)}}\right)^{\circ}$, where we use the notation of (3.3.5). Note that the kernel of

$$
\mathscr{A}\left(G_{\mathbb{Z}_{(p)}}\right)^{\circ} \stackrel{\sim}{\longrightarrow} \mathscr{A}\left(G_{\mathbb{Z}_{(p)}}^{\text {der }}\right)^{\circ} \rightarrow \mathscr{A}\left(G_{2, \mathbb{Z}_{(p)}}^{\text {der }}\right)^{\circ} \hookrightarrow \mathscr{A}\left(G_{2, \mathbb{Z}_{(p)}}\right)
$$

is easily seen to be finite, so the quotient makes sense.

Proposition (3.3.10). There is an isomorphism of $E^{p}$-schemes with continuous $G_{2}\left(\mathbb{A}_{f}^{p}\right)$-action

$$
\operatorname{Sh}_{K_{2, p}}\left(G_{2}, X_{2}\right) \stackrel{\sim}{\longrightarrow}\left[\mathscr{A}\left(G_{2, \mathbb{Z}_{(p)}}\right) \times \operatorname{Sh}_{K_{p}}(G, X)^{+}\right] / \mathscr{A}\left(G_{\mathbb{Z}_{(p)}}\right)^{\circ}
$$

which is compatible with the action of $\mathrm{Gal}\left(E^{p} / E\right)$ when the right-hand side is equipped with the action of

$$
\mathscr{A}\left(G_{2, \mathbb{Z}_{(p)}}\right) *_{\mathscr{A}\left(G_{\mathbb{Z}_{(p)}}\right)} \circ \mathscr{E}\left(G_{\mathbb{Z}_{(p)}}^{\text {der }}\right) \stackrel{\sim}{\longrightarrow} \mathscr{A}\left(G_{2, \mathbb{Z}_{(p)}}\right) \times \operatorname{Gal}\left(E^{p} / E\right) .
$$

Proof. We will use the notation introduced in the proof of (3.3.7). By De 3, 2.7.11, 2.7.13] there is an isomorphism of $\bar{E}$-schemes with continuous $G_{2}\left(\mathbb{A}_{f}\right)$-action

$$
\begin{aligned}
\operatorname{Sh}\left(G_{2}, X_{2}\right) \stackrel{\sim}{\longrightarrow}\left[\mathscr{A}\left(G_{2}\right) \times \operatorname{Sh}(G, X)^{+}\right] / \mathscr{A}(G)^{\circ} \\
\stackrel{\sim}{\longrightarrow}\left[\tilde{\mathscr{A}}\left(G_{2, \mathbb{Z}_{(p)}}\right) \times \operatorname{Sh}(G, X)^{+}\right] / \tilde{\mathscr{A}}\left(G_{\mathbb{Z}_{(p)}}\right)^{\circ}
\end{aligned}
$$

compatible with the action of $\operatorname{Gal}(\bar{E} / E)$ on both sides. Here the second isomorphism follows from (3.3.3). The $\operatorname{Gal}(\bar{E} / E)$ actions on the second and third terms are induced by the actions of $\mathscr{E}\left(G^{\text {der }}\right)$ and $\tilde{\mathscr{E}}\left(G_{\mathbb{Z}_{(p)}}^{\text {der }}\right)$, respectively, which are defined as in (3.3.9).

Dividing by $G_{2}\left(\mathbb{Z}_{p}\right)$ induces $\operatorname{Gal}(\bar{E} / E)$-compatible isomorphisms of $\bar{E}$-schemes with continuous $G_{2}\left(\mathbb{A}_{f}^{p}\right)$-action:

$$
\begin{aligned}
\operatorname{Sh}_{K_{2, p}}\left(G_{2}, X_{2}\right) \stackrel{\sim}{\longrightarrow}\left[\mathscr{A}\left(G_{2, \mathbb{Z}_{(p)}}\right)\right. & \left.\times \operatorname{Sh}(G, X)^{+} /\left(\mathscr{A}(G)^{\circ} \cap G\left(\mathbb{Z}_{p}\right)\right)\right] / \mathscr{A}\left(G_{\mathbb{Z}_{(p)}}\right)^{\circ} \\
& \stackrel{\sim}{\longrightarrow}\left[\mathscr{A}\left(G_{2, \mathbb{Z}_{(p)}}\right) \times \operatorname{Sh}_{K_{p}}(G, X)^{+}\right] / \mathscr{A}\left(G_{\mathbb{Z}_{(p)}}\right)^{\circ} .
\end{aligned}
$$

Finally, descending the first and last term to $E^{p}$, gives the isomorphism of the proposition. 
(3.4) Integral models II. Now assume that, if $p=2$, then $(G, X)$ satisfies the condition (2.3.4). In particular, we have the integral canonical model $\mathscr{S}_{K}(G, X)$ over $\mathcal{O}_{(v)}$, constructed in (2.3.8) as the normalization of the closure of

$$
\mathrm{Sh}_{K}(G, X) \hookrightarrow \mathscr{S}_{K^{\prime}}\left(\mathrm{GSp}, S^{ \pm}\right)
$$

We again denote by $(\mathcal{A}, \lambda)$ the pullback to $\mathscr{S}_{K}(G, X)$ of the universal polarized abelian scheme over $\mathscr{S}_{K^{\prime}}\left(\mathrm{GSp}, S^{ \pm}\right)$.

Lemma (3.4.1). The embedding of (3.2.2) induces an embedding

$$
Z \hookrightarrow \underline{\operatorname{Aut}}_{\mathbb{Q}} \mathcal{A} \text {. }
$$

Proof. This follows from the fact that any endomorphism of $\left.\mathcal{A}\right|_{\mathrm{Sh}_{K}(G, X)}$ extends to an endomorphism over $\mathscr{S}_{K}(G, X)$ [FC, I 2.7]. Note that this shows that the group Aut $_{\mathbb{Q}} \mathcal{A}$ in the statement of the lemma agrees with the one denoted by the same symbol in (3.1.2).

(3.4.2) Let $\mathcal{B}$ be an abelian scheme up to isogeny over an $\mathcal{O}_{(v)}$ scheme $T$. Using the notation of $(2.3 .3)$, we write

$$
\widehat{V}^{p}(\mathcal{B})_{\mathbb{Z}_{(p)}}=\widehat{V}^{p}(\mathcal{B}) \otimes_{\mathbb{Z}} \mathbb{Z}_{(p)}=\widehat{V}^{p}(\mathcal{B}) \otimes_{\mathbb{Z}} \mathbb{Q}
$$

Note that this is functorial for quasi-isogenies.

Now consider the category obtained from the category of abelian schemes over $T$, by tensoring the Hom groups by $\otimes \mathbb{Z}_{(p)}$. An object in this category will be called an abelian scheme up to prime to $p$ isogeny. An isomorphism in this category will be called a $p^{\prime}$-quasi-isogeny.

Now fix a $\mathbb{Z}$-lattice $V_{\mathbb{Z}} \subset V$ as in (2.3.2). By (2.3.3), any $x \in \mathrm{Sh}_{K^{\prime}}\left(\mathrm{GSp}, S^{ \pm}\right)(T)$ gives rise to a triple $\left(\mathcal{A}_{x}, \lambda, \varepsilon_{K^{\prime}}^{p}\right)$, consisting of an abelian scheme, a polarization and a section of $\Gamma\left(T, \underline{\operatorname{Isom}}\left(V_{\widehat{\mathbb{Z}}^{p}}, \widehat{V}^{p}\left(\mathcal{A}_{x}\right)\right) / K^{\prime p}\right)$. We will view $\mathcal{A}_{x}$ as an abelian scheme up to prime to $p$ isogeny, $\lambda$ as a weak polarization, and $\varepsilon_{K^{\prime}}^{p}$ as a section of $\Gamma\left(T, \underline{\operatorname{Isom}}\left(V_{\mathbb{A}_{f}^{p}}, \widehat{V}^{p}\left(\mathcal{A}_{x}\right)_{\mathbb{Z}_{(p)}}\right) / K^{\prime p}\right)$. (When viewed in this way, the triple depends, up to isomorphism, only on $V_{\mathbb{Z}_{(p)}}$ and not on $V_{\mathbb{Z}}$.)

If $x \in \operatorname{Sh}_{K}(G, X)(T)$, then, as in (3.2.4), we may promote $\varepsilon_{K^{\prime}}^{p}$ to a section

$$
\varepsilon^{p} \in \Gamma\left(T, \underline{\operatorname{Isom}}\left(V_{\mathbb{A}_{f}^{p}}, \widehat{V}^{p}\left(\mathcal{A}_{x}\right)_{\mathbb{Z}_{(p)}}\right) / K^{p}\right) .
$$

Fix a point $x$ as above. Let $\gamma \in G^{\text {ad }}(\mathbb{Q})$ and let $\mathcal{P}$ be the corresponding $Z$-torsor, as in (3.2.4).

Lemma (3.4.3). Fix a finite Galois extension $F / \mathbb{Q}$ with $\mathcal{P}(F)$ non-empty and let $\tilde{\gamma} \in \mathcal{P}(F)$. For $\sigma \in \operatorname{Gal}(F / \mathbb{Q})$, let $c_{\tilde{\gamma}}(\sigma)=\sigma(\tilde{\gamma}) \tilde{\gamma}^{-1} \in Z(F)$, and let

$$
\iota_{\tilde{\gamma}}: \mathcal{A}_{x}^{\mathcal{P}} \otimes_{\mathbb{Q}} F \stackrel{\sim}{\longrightarrow} \mathcal{A}_{x} \otimes_{\mathbb{Q}} F
$$

be the isomorphism obtained by specializing the map of (3.1.1)(2) at $\tilde{\gamma}$. Then

(1) $\sigma \circ \iota_{\tilde{\gamma}} \circ \sigma^{-1}=c_{\tilde{\gamma}}(\sigma)^{-1} \cdot \iota_{\tilde{\gamma}}$.

(2) The composite (defined étale locally on $T$ )

$$
\begin{gathered}
V \otimes_{\mathbb{Q}} \mathbb{A}_{f}^{p} \otimes_{\mathbb{Q}} F \stackrel{\tilde{\gamma}^{-1}}{\rightarrow} V \otimes_{\mathbb{Q}} \mathbb{A}_{f}^{p} \otimes_{\mathbb{Q}} F \stackrel{\varepsilon^{p}}{\rightarrow} \widehat{V}^{p}\left(\mathcal{A}_{x}\right) \otimes_{\mathbb{Q}} F \stackrel{{ }^{i} \bar{\gamma}^{-1}}{\rightarrow} \widehat{V}^{p}\left(\mathcal{A}_{x}^{\mathcal{P}}\right) \otimes_{\mathbb{Q}} F \\
\text { is } \operatorname{Gal}(F / \mathbb{Q}) \text {-invariant and induces a section } \\
\varepsilon^{p, \mathcal{P}} \in \Gamma\left(T, \underline{\operatorname{Isom}}\left(V_{\mathbb{A}_{f}^{p}}, \widehat{V}^{p}\left(\mathcal{A}_{x}^{\mathcal{P}}\right)_{\mathbb{Z}_{(p)}}\right) / \gamma K^{p} \gamma^{-1}\right) .
\end{gathered}
$$


Proof. It suffices to prove the lemma when $\mathcal{A}_{x}=\mathcal{A}$ is the universal abelian scheme over $\mathscr{S}_{K}(G, X)$. Since $\mathscr{S}_{K}(G, X)$ is flat over $\mathcal{O}_{(v)}$ it suffices to check (1) and (2) over $\operatorname{Sh}_{K}(G, X)$. In this case the lemma is a formal consequence of (3.2.5).

(3.4.4) In the following we will consider the triple $\left(\mathcal{A}_{x}^{\mathcal{P}}, \lambda^{\mathcal{P}}, \varepsilon^{p, \mathcal{P}}\right)$. Note that $\mathcal{A}_{x}^{\mathcal{P}}$ is defined only in the isogeny category of abelian $T$-schemes. If $\left(\mathcal{A}^{\prime}, \lambda^{\prime}, \varepsilon^{p \prime}\right)$ is a triple of the form discussed in (3.4.2), so that $\mathcal{A}^{\prime}$ is an abelian scheme up to prime to $p$ isogeny, we will say that $\left(\mathcal{A}^{\prime}, \lambda^{\prime}, \varepsilon^{p \prime}\right)$ is isogenous to $\left(\mathcal{A}_{x}^{\mathcal{P}}, \lambda^{\mathcal{P}}, \varepsilon^{p, \mathcal{P}}\right)$ if these triples become isomorphic when one considers $\mathcal{A}^{\prime}$ as an abelian scheme up to isogeny.

Lemma (3.4.5). The action of $\mathscr{A}(G)$ on $\operatorname{Sh}(G, X)$ induces an action of $\mathscr{A}\left(G_{\mathbb{Z}_{(p)}}\right)$ on $\mathscr{S}_{K_{p}}(G, X)$. More precisely, if $\left(h, \gamma^{-1}\right) \in \mathscr{A}\left(G_{\mathbb{Z}_{(p)}}\right)$, then the above action induces a map

$$
\left(h, \gamma^{-1}\right): \mathscr{S}_{K}(G, X) \rightarrow \mathscr{S}_{\gamma K \gamma^{-1}}(G, X)
$$

such that the triple attached to $\left(h, \gamma^{-1}\right)(x)$ is isogenous to $\left(\mathcal{A}_{x}^{\mathcal{P}}, \lambda^{\mathcal{P}}, \varepsilon^{p, \mathcal{P}} \circ \tilde{\gamma} h \tilde{\gamma}^{-1}\right)$, where we use the notation of (3.4.3).

Proof. Since $\gamma \in G\left(\mathbb{Z}_{(p)}\right)$, we have $\gamma K_{p} \gamma^{-1} \subset K_{p}$, so the action of $\mathscr{A}(G)$ on $\operatorname{Sh}(G, X)$ induces an action of $\mathscr{A}\left(G_{\mathbb{Z}_{(p)}}\right)$ on $\operatorname{Sh}_{K_{p}}(G, X)$. More precisely, $\left(h, \gamma^{-1}\right)$ induces a map

$$
\operatorname{Sh}_{K}(G, X) \rightarrow \operatorname{Sh}_{\gamma K \gamma^{-1}}(G, X) .
$$

The extension property implies that this map extends to a map

$$
\mathscr{S}_{K}(G, X) \rightarrow \mathscr{S}_{\gamma K \gamma^{-1}}(G, X) \text {. }
$$

By (3.2.6) and [FC] I 2.7], the triple attached to $\left(h, \gamma^{-1}\right)(x)$ is $\left(\mathcal{A}_{x}^{\mathcal{P}}, \lambda^{\mathcal{P}},\left(\varepsilon^{p} \circ h\right)^{\mathcal{P}}\right)$. As $\left(\varepsilon^{p} \circ h\right)^{\mathcal{P}}=\varepsilon^{p, \mathcal{P}} \circ \tilde{\gamma} h \tilde{\gamma}^{-1}$ the lemma follows.

Proposition (3.4.6). The group

$$
\Delta\left(G, G^{\mathrm{ad}}\right)=\operatorname{ker}\left(\mathscr{A}\left(G_{\mathbb{Z}_{(p)}}\right) \rightarrow \mathscr{A}\left(G_{\mathbb{Z}_{(p)}}^{\mathrm{ad}}\right)\right)
$$

acts freely on $\mathscr{S}_{K_{p}}(G, X)$.

Proof. Let $\left(h, \gamma^{-1}\right) \in \Delta\left(G, G^{\text {ad }}\right)$ with $h \in G\left(\mathbb{A}_{f}^{p}\right)$ and $\gamma \in G^{\text {ad }}\left(\mathbb{Z}_{(p)}\right)^{+}$. As usual, we denote by $\mathcal{P}$ the $Z$-torsor associated to $\gamma$, and we fix a Galois extension $F / \mathbb{Q}$ and a point $\tilde{\gamma} \in \mathcal{P}(F)$.

Let $x \in \mathscr{S}_{K_{p}}(G, X)(T)$, where $T$ is the spectrum of an algebraically closed field, and suppose that $\left(h, \gamma^{-1}\right)$ fixes $x$. Write $\left(\mathcal{A}_{x}, \lambda, \varepsilon^{p}\right)$ for the corresponding triple introduced in (3.4.2). Then by (3.4.5), for every compact open subgroup $K^{p} \subset G\left(\mathbb{A}_{f}^{p}\right)$ there exists a quasi-isogeny $\alpha=\alpha\left(K^{p}\right): \mathcal{A}_{x} \stackrel{\sim}{\longrightarrow} \mathcal{A}_{x}^{\mathcal{P}}$ respecting polarizations, and such that the left-hand square of the following diagram (defined étale locally on $T$ ) commutes modulo $K^{p}$ (that is, up to multiplication by an element of $K^{p}$ on the bottom left-hand corner):

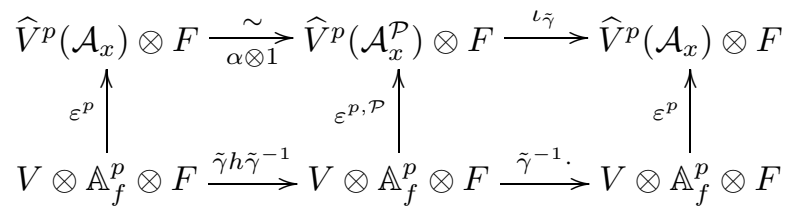

while the right square commutes by the definition of $\varepsilon^{p, \mathcal{P}}$. 
For $K^{p}$ sufficiently small, the map $\alpha\left(K^{p}\right)$ is unique. Hence if $K^{p}$ is sufficiently small, then $\alpha$ does not depend on $K^{p}$, and we may assume that (3.4.7) commutes.

Note that the composite of the maps in the lower row of (3.4.7) is $h \tilde{\gamma}^{-1}$. Since $\left(h, \gamma^{-1}\right) \in \Delta\left(G, G^{\text {ad }}\right)$, we have $h \tilde{\gamma}^{-1} \in Z\left(\mathbb{A}_{f}^{p} \otimes F\right)$. Hence

$$
\iota_{\tilde{\gamma}} \circ \alpha \in Z\left(\mathbb{A}_{f}^{p} \otimes F\right) \cap\left(\underline{\mathrm{Aut}}_{\mathbb{Q}} \mathcal{A}_{x}\right)(F)=Z(F) \subset\left(\underline{\mathrm{Aut}}_{\mathbb{Q}} \mathcal{A}_{x}\right)\left(\mathbb{A}_{f}^{p} \otimes F\right) .
$$

Hence $h \tilde{\gamma}^{-1} \in Z(F)$. Writing

$$
\tilde{\gamma}=\left(h^{-1} \tilde{\gamma}\right) \cdot\left(\tilde{\gamma}^{-1} h \tilde{\gamma}\right)
$$

and noting that $\tilde{\gamma}^{-1} h \tilde{\gamma}$ is $\operatorname{Gal}(F / \mathbb{Q})$-invariant, we see that the cocycle $c_{\tilde{\gamma}}(\sigma)$ is trivial.

It follows that $\mathcal{P}$ is trivial. Let $\mathcal{P}_{\mathbb{Z}_{(p)}}$ denote the fibre of $G_{\mathbb{Z}_{(p)}} \rightarrow G_{\mathbb{Z}_{(p)}}^{\text {ad }}$ over $\gamma$. Then $\mathcal{P}_{\mathbb{Z}_{(p)}}$ is a $Z_{\mathbb{Z}_{(p)}}$-torsor with generic fibre $\mathcal{P}$. It follows from (3.4.8) below that $\mathcal{P}_{\mathbb{Z}_{(p)}}$ is the trivial torsor, so that we may assume that $\tilde{\gamma} \in G\left(\mathbb{Z}_{(p)}\right)_{+}$, and after replacing $h$ by $h \tilde{\gamma}^{-1}$, that $\tilde{\gamma}=1$.

In particular, we may identify $\mathcal{A}_{x}$ and $\mathcal{A}_{x}^{\mathcal{P}}$ and regard the latter as an abelian scheme up to prime to $p$ isogeny. Since $(h, 1)$ fixes $x$, there exists a $p^{\prime}$-quasi-isogeny $\alpha: \mathcal{A}_{x} \stackrel{\sim}{\longrightarrow} \mathcal{A}_{x}$ making the left square in (3.4.7) commute 15 Moreover, we have already observed that $h \in Z(\mathbb{Q})$ (as we can now take $F=\mathbb{Q}$ above).

It remains to show that, via (3.4.1), an element $z \in Z(\mathbb{Q})$ induces a $p^{\prime}$-isogeny $\mathcal{A}_{x} \rightarrow \mathcal{A}_{x}$ if and only if $z \in Z\left(\mathbb{Z}_{(p)}\right)$. Since the degree of an isogeny of abelian schemes is a locally constant function, it suffices to check this over the generic fibre of $\mathscr{S}_{K}(G, X)$, and then on complex points. Then $z$ induces a $p^{\prime}$-isogeny if and only if

$$
z \in Z(\mathbb{Q}) \cap K_{p}^{\prime}=Z(\mathbb{Q}) \cap K_{p}=Z(\mathbb{Q}) \cap G\left(\mathbb{Z}_{(p)}\right)=Z\left(\mathbb{Z}_{(p)}\right) .
$$

Lemma (3.4.8). Let $\mathcal{P}_{\mathbb{Z}_{(p)}}$ be a $Z_{\mathbb{Z}_{(p)}}$-torsor. If $\mathcal{P}=\left.\mathcal{P}_{\mathbb{Z}_{(p)}}\right|_{\text {Spec } \mathbb{Q}}$ is a trivial $Z$ torsor, then $\mathcal{P}_{\mathbb{Z}_{(p)}}$ is a trivial $Z_{\mathbb{Z}_{(p)}}$-torsor.

Proof. In order not to overload notation, we write $Z$ for $Z_{\mathbb{Z}_{(p)}}$. By [DG, XII, Thm. 4.7], $Z$ is of multiplicative type and hence is an extension of a finite flat multiplicative group scheme by a torus $Z^{0} \subset Z$. Now consider the commutative diagram

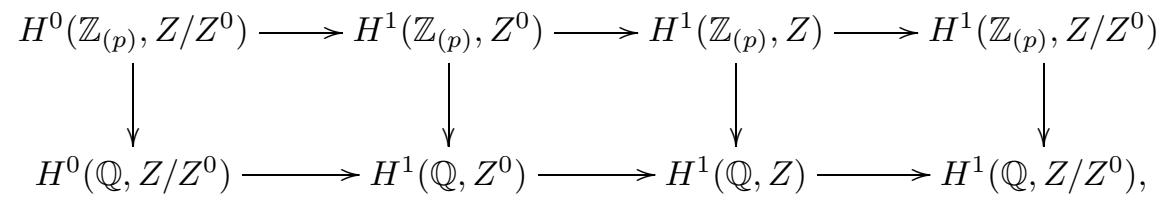

where the $H^{*}$ means cohomology in the fppf topology of the spectrum of the indicated ring.

For a finite flat $\mathbb{Z}_{(p)}$-scheme the $\mathbb{Z}_{(p)}$-points are in bijection with the $\mathbb{Q}$-points. Hence the map on the left is a bijection and the map on the right is an injection. To prove the lemma, we have to show that the third map is an injection. By the five lemma it suffices to prove that the second map is an injection.

To see this, note that the kernel of this map is the cokernel of

$$
Z^{0}(\mathbb{Q}) / Z^{0}\left(\mathbb{Z}_{(p)}\right) \rightarrow H_{(p)}^{1}\left(\mathbb{Z}_{(p)}, Z^{0}\right),
$$

\footnotetext{
${ }^{15}$ Note that when $\tilde{\gamma} \neq 1$, it does not make sense to require that $\alpha: \mathcal{A}_{x} \stackrel{\sim}{\longrightarrow} \mathcal{A}_{x}^{\mathcal{P}}$ be a $p^{\prime}$-quasiisogeny, since $\mathcal{A}_{x}^{\mathcal{P}}$ is defined only up to isogeny.
} 
where $H_{(p)}^{1}$ denotes cohomology with support in $(p)$. By Lang's lemma we have $H^{1}\left(\mathbb{Z}_{p}, Z^{0}\right)=0$, so that

$$
H_{(p)}^{1}\left(\mathbb{Z}_{(p)}, Z^{0}\right)=H_{(p)}^{1}\left(\mathbb{Z}_{p}, Z^{0}\right) \stackrel{\sim}{\longrightarrow} Z^{0}\left(\mathbb{Q}_{p}\right) / Z^{0}\left(\mathbb{Z}_{p}\right) .
$$

Finally, by $(2.2 .6), Z^{0}(\mathbb{Q}) / Z^{0}\left(\mathbb{Z}_{(p)}\right) \rightarrow Z^{0}\left(\mathbb{Q}_{p}\right) / Z^{0}\left(\mathbb{Z}_{p}\right)$ is a surjection and the lemma follows.

(3.4.9) We are now ready to prove the main theorem of this section. Let $\left(G_{2}, X_{2}\right)$ be as in (3.3.6). Thus, $G_{2, \mathbb{Z}_{(p)}}$ is a reductive $\mathbb{Z}_{(p)}$-group equipped with a central isogeny $G_{\mathbb{Z}_{(p)}}^{\text {der }} \rightarrow G_{2, \mathbb{Z}_{(p)}}^{\text {der }}$ which induces an isomorphism $\left(G^{\text {ad }}, X^{\text {ad }}\right) \stackrel{\sim}{\longrightarrow}\left(G_{2}^{\text {ad }}, X_{2}^{\text {ad }}\right)$, and $K_{2, p}=G_{2, \mathbb{Z}_{(p)}}\left(\mathbb{Z}_{p}\right) \subset G_{2}\left(\mathbb{Q}_{p}\right)$ is a hyperspecial subgroup. We will denote by $E_{2}=E\left(G_{2}, X_{2}\right) \subset E$ the reflex field of $\left(G_{2}, X_{2}\right)$, and by $\mathcal{O}_{2}$ its ring of integers.

Theorem (3.4.10). Let $v \mid p$ be a prime of $\mathcal{O}_{2}$. Then the tower of $E_{2}$-schemes $\mathrm{Sh}_{K_{2, p}}\left(G_{2}, X_{2}\right)$ admits a $G_{2}\left(\mathbb{A}_{f}^{p}\right)$-equivariant extension to a tower of $\mathcal{O}_{2,(v)}$-schemes

$$
\mathscr{S}_{K_{2, p}}\left(G_{2}, X_{2}\right)=\lim _{K_{2}^{p}} \mathscr{S}_{K_{2, p} K_{2}^{p}}\left(G_{2}, X_{2}\right)
$$

which has the extension property.

Proof. For each $K=K_{p} K^{p}$ with $K^{p} \subset G\left(\mathbb{A}_{f}^{p}\right)$ a compact open subgroup, let $K^{\prime}=K_{p}^{\prime} K^{\prime p}$ be as in (2.3.7), and denote by $\mathscr{S}_{K}(G, X)$ the normalization of the closure of

$$
\mathrm{Sh}_{K}(G, X) \hookrightarrow \mathscr{S}_{K^{\prime}}\left(\mathrm{GSp}, S^{ \pm}\right)_{\mathcal{O}_{(p)}} .
$$

Note that the $\mathcal{O}_{(v)}$-scheme denoted by the same symbol in (2.3.8) is the localization of $\mathscr{S}_{K}(G, X)$ at $(v)$. By $(2.3 .8)$,

$$
\mathscr{S}_{K_{p}}(G, X)=\lim _{K^{p}} \mathscr{S}_{K}(G, X)
$$

is a $G\left(\mathbb{A}_{f}^{p}\right)$-equivariant tower of smooth $\mathcal{O}_{(p)}$-schemes having the extension property. In particular, the action of $\mathscr{A}\left(G_{\mathbb{Z}_{(p)}}\right)$ on $\operatorname{Sh}_{K_{p}}(G, X)$ extends to $\mathscr{S}_{K_{p}}(G, X)$, as already noted in (3.4.5).

As in (3.3.6), let $E^{p} \subset \bar{E}$ be the maximal subfield in which $p$ is unramified, and write $\mathcal{O}_{(p)}^{p}$ for its ring of integers localized at $(p)$. Denote by $\mathscr{S}_{K}(G, X)^{+}$the closure of $\operatorname{Sh}_{K}(G, X)^{+}$in $\mathscr{S}_{K}(G, X) \otimes_{\mathcal{O}_{(p)}} \mathcal{O}_{(p)}^{p}$. Set

$$
\mathscr{S}_{K_{p}}(G, X)^{+}=\varliminf_{K^{p}} \mathscr{S}_{K}(G, X)^{+} .
$$

The action of $\mathscr{A}\left(G_{\mathbb{Z}_{(p)}}\right) \times \operatorname{Gal}\left(E^{p} / E\right)$ on $\mathscr{S}_{K_{p}}(G, X) \otimes_{E} E^{p}$ induces an action of $\mathscr{E}\left(G_{\mathbb{Z}_{(p)}}^{\text {der }}\right)$ on $\mathscr{S}_{K_{p}}(G, X)^{+}$. Write

$$
\mathscr{S}_{K_{2, p}}\left(G_{2}, X_{2}\right)=\left[\mathscr{A}\left(G_{2, \mathbb{Z}_{(p)}}\right) \times \mathscr{S}_{K_{p}}(G, X)^{+}\right] / \mathscr{A}\left(G_{\mathbb{Z}_{(p)}}\right)^{\circ} .
$$

Since $\operatorname{ker}\left(\mathscr{A}\left(G_{\mathbb{Z}_{(p)}}\right)^{\circ} \rightarrow \mathscr{A}\left(G_{2, \mathbb{Z}_{(p)}}\right)\right)$ is a subgroup of $\Delta\left(G, G^{\text {ad }}\right)$, (3.4.6) implies that (3.4.11) is a tower of smooth $\mathcal{O}_{(p)}^{p}$-schemes. The $\mathscr{E}\left(G_{\mathbb{Z}_{(p)}}^{\text {der }}\right)$-action on $\mathscr{S}_{K_{p}}(G, X)^{+}$ induces a $\mathscr{A}\left(G_{2, \mathbb{Z}_{(p)}}\right) \times \operatorname{Gal}\left(E^{p} / E\right)$-action on (3.4.11). By (3.3.10), the generic fibre of (3.4.11) may be $\mathscr{A}\left(G_{2, \mathbb{Z}_{(p)}}\right) \times \operatorname{Gal}\left(E^{p} / E\right)$-equivariantly identified with the $E^{p}$ scheme $\operatorname{Sh}_{K_{2, p}}\left(G_{2}, X_{2}\right)$. Hence (3.4.11) descends to a tower of smooth $\mathcal{O}_{(p)}$-schemes, again denoted $\mathscr{S}_{K_{2, p}}\left(G_{2}, X_{2}\right)$ whose generic fibre may be $G_{2}\left(\mathbb{A}_{f}^{p}\right)$-equivariantly identified with $\operatorname{Sh}_{K_{2, p}}(G, X)$. That this tower has the extension property follows from the argument in [Mo, Prop. 3.21.4]. 
Finally, since $\operatorname{Sh}_{K_{2, p}}\left(G_{2}, X_{2}\right)$ has a canonical model defined over $E_{2}$, the tower of $E$-schemes $\operatorname{Sh}_{K_{2, p}}\left(G_{2}, X_{2}\right)$ is equipped with a descent datum relative to $E / E_{2}$. The extension property implies that this induces a descent datum on $\mathscr{S}_{K_{2, p}}\left(G_{2}, X_{2}\right)$ relative to $\mathcal{O}_{(p)} / \mathcal{O}_{2,(p)}$. Since descent for a quasi-projective scheme relative to a finite flat morphism is effective, $\mathscr{S}_{K_{2, p}}\left(G_{2}, X_{2}\right)$ descends to a $G_{2}\left(\mathbb{A}_{f}^{p}\right)$-equivariant tower of smooth $\mathcal{O}_{2,(p)}$-schemes having the extension property.

(3.4.12) Let $\left(G_{2}, X_{2}\right)$ be a Shimura datum of abelian type and $K_{2}=K_{2, p} K_{2}^{p}$ $\subset G_{2}\left(\mathbb{A}_{f}\right)$ a compact open subgroup with $K_{2, p}$ hyperspecial. By $(3.4 .10)$, $\operatorname{Sh}_{K_{2, p}}\left(G_{2}, X_{2}\right)$ has a canonical integral model at any prime $v \mid p$ of $E\left(G_{2}, X_{2}\right)$, provided $K_{2, p}$ can be constructed starting with a hyperspecial subgroup of $G\left(\mathbb{Q}_{p}\right)$, where $(G, X)$ is a Shimura datum of Hodge type as in (3.3.6). It remains to show that we can always choose $(G, X)$ so that this condition is satisfied 16

To do this we recall Deligne's classification of Shimura data of abelian type.

Lemma (3.4.13). Let $(H, Y)$ be a Shimura datum of abelian type with $H$ an adjoint group. Then there exists a central isogeny $H^{\sharp} \rightarrow H$ such that

(1) If $(G, X)$ is a Shimura datum of Hodge type such that $\left(G^{\mathrm{ad}}, X^{\mathrm{ad}}\right)$ is isomorphic to $(H, Y)$, then $G^{\text {der }}$ is a quotient of $H^{\sharp}$.

(2) There exists a Shimura datum of Hodge type $(G, X)$ such that $\left(G{ }^{\text {ad }}, X^{\text {ad }}\right)$ is isomorphic to $(H, Y)$ and $G^{\text {der }}=H^{\sharp}$.

(3) If $H$ is quasi-split and unramified at a prime $p$, then $(G, X)$ in (2) can be chosen so that $G$ is quasi-split and unramified at $p$.

Proof. The first two claims are implicit in De 3], but we explain how to reduce them to the results proved by Deligne in loc. cit.

Let $(H, Y)=\left(H_{1}, Y_{1}\right) \times \cdots \times\left(H_{r}, Y_{r}\right)$ with $H_{i}$ adjoint and $\mathbb{Q}$-simple. For each $i=1, \ldots, r$, let $H_{i, v}$, for $v=1, \ldots, j_{i}$, denote the simple factors of $H_{i}$ over $\mathbb{R}$. Write $\tilde{H}_{i, v}$ (resp. $\tilde{H}_{i}$, resp. $\tilde{H}$ ) for the universal cover of $H_{i, v}$, (resp. $H_{i}$, resp. $H$ ). Let $H_{i}^{\sharp}$ be the cover of $H_{i}$ constructed in [De 3, 2.3.7, 2.3.8]. Then $H_{i}^{\sharp}$ satisfies (1) with $\left(H_{i}, Y_{i}\right)$ in place of $(H, Y)$. (See the remark at the end of [De 3, 2.3.7].) We fix $h \in Y$ and a fractional lifting $\tilde{h}: \mathbb{S} \rightarrow \tilde{H}_{\mathbb{R}}$ of $h$. We denote by $\tilde{h}_{i, v}: \mathbb{S} \rightarrow \tilde{H}_{i, v}$ the components of $\tilde{h}$. Finally we denote by $\tilde{\mu}_{h}$ and $\tilde{\mu}_{h_{i, v}}$ the fractional cocharacters attached to $\tilde{h}$ and $\tilde{h}_{i, v}$, respectively [De 3, 1.2 .4 ].

Suppose that $(G, X)$ is as in (1) and consider an embedding of Shimura data $(G, X) \hookrightarrow\left(\mathrm{GSp}(V), S^{ \pm}\right)$. We think of $V$ as an $\tilde{H}$-representation via $\tilde{H} \rightarrow G^{\text {der }} \subset G$. Let $W \subset V_{\mathbb{C}}$ be an irreducible $\tilde{H}$-subrepresentation. Write $W=\bigotimes_{i, v} W_{i, v}$, where $W_{i, v}$ is an irreducible complex representation of $\tilde{H}_{i, v}$. If $W_{i, v}$ is non-trivial, let $w_{i, v}$ be the highest weight of $W_{i, v}$ as an $\tilde{H}_{i, v}$-representation, and for each $i, v$, let $S_{i, v}$ be the set of $w_{i, v}$ with $W_{i, v}$ non-trivial as $W$ runs over irreducible factors of $V_{\mathbb{C}}$. For each $i$ the absolute Galois group of $\mathbb{Q}$ permutes the $H_{i, v}$ transitively. If a Galois element sends $H_{i, v}$ to $H_{i, v^{\prime}}$, then it maps $S_{i, v}$ bijectively onto $S_{i, v^{\prime}}$. Note that the $S_{i, v}$ are non-empty since $V$ is a faithful representation of $G$.

As in $\left[\right.$ De 3, 1.3.5], $\tilde{\mu}_{h}$ acting on $W$ has two weights, $a, a+1$, for some $a \in \mathbb{Q}$. Hence for each $W$ there is at most one pair $(i, v)$ such that the action of $\tilde{\mu}_{h_{i, v}}$ on $W_{i, v}$ is non-trivial. For such a pair the weights of $\tilde{\mu}_{h_{i, v}}$ on $W_{i, v}$ have the form $a, a+1$, and hence $w_{i, v}$ is a fundamental weight which appears in the table of [De 3,

\footnotetext{
${ }^{16}$ At least when $p>2$. When $p=2$, whether $(G, X)$ can be made to satisfy (2.3.4) depends on $\left(G_{2}, X_{2}\right)$.
} 
1.3.9]. For each $i$ at least one of the factors $H_{i, v}$ is non-compact. Hence the remark on transitivity of the Galois action shows that all the $S_{i, v}$ consist of fundamental weights which appear in loc. cit.

Now for each $i$ let $\mathscr{S}_{i}$ be the union of the $S_{i, v}, v=1, \ldots, j_{i}$. where we now consider the elements of $S_{i, v}$ as fundamental weights of $H_{i}$. Then the set $\mathscr{S}_{i}$ satisfies 17 the conditions (b), (c), (d) of De 3, 2.3.7]: It is a non-empty, Galois stable, set of fundamental weights of $H_{i}$ such that any element which is a fundamental weight for a non-compact $H_{i, v}$ appears in De 3. 1.3.9]. Hence, by definition, the action of $\tilde{H}_{i}$ on $V$ factors through $H_{i}^{\sharp}$.

This proves that $H^{\sharp}$ satisfies (1), and (2) follows from De 3. 2.3.10]. Finally, to see (3), note that $H_{i}$ has the form $\operatorname{Res}_{F / \mathbb{Q}} H_{i}^{s}$ with $F$ a totally real field, and $H_{i}^{s}$ absolutely simple [De 3, 2.3.4]. If $H$ is quasi-split and unramified at $p$, then $p$ is unramified in $F$. Now (3) follows from the proof of [De 3, 2.3.10] since the field $K$ in loc. cit can be chosen to be an extension of $F$ which is unramified at primes above $p$.

Corollary (3.4.14). Let $\left(G_{2}, X_{2}\right)$ be a Shimura datum of abelian type, and $K_{2}=$ $K_{2, p} K_{2}^{p} \subset G_{2}\left(\mathbb{A}_{f}\right)$ compact open with $K_{2, p}$ hyperspecial. If $p>218$ and $v \mid p$ is a prime of the ring of integers $\mathcal{O}_{2} \subset E\left(G_{2}, X_{2}\right)$, then the tower of $E_{2}$-schemes $\mathrm{Sh}_{K_{2, p}}\left(G_{2}, X_{2}\right)$ admits a $G_{2}\left(\mathbb{A}_{f}^{p}\right)$-equivariant extension to a tower of $\mathcal{O}_{2,(v)}$-schemes

$$
\mathscr{S}_{K_{2, p}}\left(G_{2}, X_{2}\right)=\lim _{K_{2}^{p}} \mathscr{S}_{K_{2, p} K_{2}^{p}}\left(G_{2}, X_{2}\right)
$$

which has the extension property.

Proof. Let $(G, X)$ be a Shimura datum of Hodge type and $G^{\text {der }} \rightarrow G_{2}^{\text {der }}$ a central isogeny inducing an isomorphism $\left(G^{\text {ad }}, X^{\text {ad }}\right) \stackrel{\sim}{\longrightarrow}\left(G_{2}^{\text {ad }}, X_{2}^{\text {ad }}\right)$. By $(3.4 .13)(1), G^{\text {der }}$, and hence $G_{2}^{\text {der }}$, is a quotient of $G^{\text {ad\# }}$. Hence by $(3.4 .13)(3)$, we may assume that $G$ is quasi-split and unramified at $p$.

Now let $G_{2, \mathbb{Z}_{(p)}}$ be a reductive group over $\mathbb{Z}_{(p)}$ with generic fibre $G_{2}$, and such that $K_{2, p}=G_{2, \mathbb{Z}_{(p)}}\left(\mathbb{Z}_{p}\right)$. The central isogeny $G^{\text {der }} \rightarrow G_{2}^{\text {der }}$ extends uniquely to a central isogeny $G_{\mathbb{Z}_{(p)}}^{\text {der }} \rightarrow G_{2, \mathbb{Z}_{(p)}}^{\text {der }}$, of reductive groups over $\mathbb{Z}_{(p)}$.

Let $Z \subset G$ denote the center of $G$. Since $G$ admits an extension to a reductive group over $\mathbb{Z}_{(p)}, Z$ admits an extension to a flat $\mathbb{Z}_{(p)}$-group $Z_{\mathbb{Z}_{(p)}}$ of multiplicative type. The kernel of $G^{\text {der }} \times Z \rightarrow G$ is a finite, multiplicative $\mathbb{Z}_{(p) \text {-group, and }}$ extends to a finite flat, multiplicative subgroup $C \subset G_{\mathbb{Z}_{(p)}}^{\text {der }} \times Z_{\mathbb{Z}_{(p)}}$. The quotient $G_{\mathbb{Z}_{(p)}}=G_{\mathbb{Z}_{(p)}}^{\text {der }} \times Z_{\mathbb{Z}_{(p)}} / C$ is a reductive group over $\mathbb{Z}_{(p)}$ with generic fibre $G$, and derived group $G_{\mathbb{Z}_{(p)}}^{\text {der }}$.

Thus the assumptions introduced at the end of (3.3.2) and in (3.3.6) are satisfied, and we may apply (3.4.10) to conclude the corollary.

(3.4.15) If $p=2$, then the conclusion of the corollary still holds provided the pair $(G, X)$ with $G$ quasi-split and unramified at $p$, which was constructed in the proof of (3.4.14), can be chosen so as to satisfy the conditions (2.3.4).

\footnotetext{
${ }^{17}$ In fact, to be strictly compatible with Deligne's notation we should take $\mathscr{S}_{i}=\{\{w\}: w \in$ $\left.S_{i, v}, v=1, \ldots j_{i}\right\}$

${ }^{18}$ But see (3.4.15) below.
} 


\section{REFERENCES}

[Bl] D. Blasius, A p-adic property of Hodge classes on abelian varieties, Motives (Seattle 1991), 293-308, Proc. Sympos. Pure Math. 55, Part 2, Amer. Math. Soc., pp. 293-308, 1994. MR:1265557 (95j:14022)

[BO] P. Berthelot, A. Ogus, F-isocrystals and de Rham cohomology. I, Invent. Math. 72 (1983), 159-199. MR700767 (85e:14025)

[Br] C. Breuil, Schémas en groupes et corps des normes (unpublished), 1998, 13 pages.

[BT] F. Bruhat, J. Tits, Groupes réductifs sur un corps local. II. Schémas en groupes. Existence d'une donnée radicielle valuée, Pub. Math. IHES 60 (1984), 197-376. MR756316 (86c:20042)

[CS] J.-L. Colliot-Thélène, J.-J. Sansuc, Fibrés quadratiques et composantes connexes réelles, Math. Ann. 244 (1979), 105-134. MR550842 (81c:14010)

[Ch] C. Chevalley, Deux théorèmes d'arithmétique, J. Math. Soc. Japan 3 (1951), 36-44. MR0044570 (13:440a)

[CSu] J.-L. Colliot-Thélène, V. Suresh, Quelques questions d'approximation faible pour les tores algébriques, Ann. Inst. Fourier 57 (2007), 273-288. MR2316239 (2008b:11047)

[De 1] P. Deligne, Travaux de Shimura, Exp. 389, Séminaire Bourbaki (1970/1971), Lecture Notes in Math. 244, Springer, pp. 123-165, 1971. MR0498581 (58:16675)

[De 2] P. Deligne, Hodge cycles on abelian varieties, Hodge cycles, motives, and Shimura varieties, Lecture Notes in Math. 900, pp. 9-100, Springer-Verlag, 1982. MR0654325 (84m:14046)

[De 3] P. Deligne, Variétés de Shimura: interprétation modulaire, et techniques de construction de modèles canoniques, Automorphic forms, representations and $L$-functions (Corvallis 1977), Proc. Sympos. Pure Math. XXXIII, Amer. Math. Soc., pp. 247-289, 1979. MR546620 (81i:10032)

[De 4] P. Deligne, Équations différentielles à points singuliers réguliers, Lecture Notes in Math. 163, Springer, 1970. MR0417174 (54:5232)

[DG] M. Demazure, A. Grothendieck, Schémas en groupes I, II, III, Lecture Notes in Math. 151153, Springer, 1962-1970. MR0274458 (43:223a) MR0274459 (43:223b) MR0274460 $(43: 223 \mathrm{c})$

[Fa] G. Faltings, Integral crystalline cohomology over very ramified valuation rings, J. Amer. Math. Soc. 12 (1999), 117-144. MR1618483 (99e:14022)

[FC] G. Faltings, C.-L. Chai, Degeneration of Abelian varieties, Ergeb. der Math. 22, Springer, 1990. MR1083353 (92d:14036)

[Fo] J.-M. Fontaine, Représentations p-adiques des corps locaux, Grothendieck Festschrift II, Prog. Math. 87, Birkhäuser, pp. 249-309, 1991. MR.1106901 (92i:11125)

[GRR] A. Grothendieck, M. Raynaud, D. Rim, Groupes de monodromie en géométrie algébrique (SGA 7 I), Lecture Notes in Math. 288, Springer, 1972. MR0354656 (50:7134)

[Ki 1] M. Kisin, Crystalline representations and F-crystals, Algebraic geometry and number theory, Progr. Math 253, Birkhäuser Boston, pp. 459-496, 2006. MR2263197|(2007j:11163)

[Ki 2] M. Kisin, Potentially semi-stable deformation rings, J. Amer. Math. Soc. 21 (2008), 513546. MR2373358 (2009c:11194)

[Ki 3] M. Kisin, Modularity of 2-adic Barsotti-Tate representations, Invent. Math. 178 (2009), 587-634. MR2551765

[Ki 4] M. Kisin, Integral canonical models of Shimura varieties (Journées Arithmétiques 2007), J. Th. Nombres Bordeaux 21 (2009), 301-312. MR.2541427

[Ko] R. Kottwitz, Points on some Shimura varieties over finite fields, J. Amer. Math. Soc. 5 (1992), 373-444. MR1124982 (93a:11053)

[La] R. Langlands, Some contemporary problems with origins in the Jugendtraum, Mathematical developments arising from Hilbert problems (De Kalb 1974), Proc. Sympos. Pure Math. XXVIII, Amer. Math. Soc., pp. 401-418, 1976. MR0437500 (55:10426)

[Me] W. Messing, The crystals associated to Barsotti-Tate groups, with applications to Abelian schemes, Lecture Notes in Math. 264, Springer, 1972. MR0347836 (50:337)

[MFK] D. Mumford, J. Fogarty, F. Kirwan, Geometric Invariant Theory, Ergeb. der Math. 34, Springer, 1994. MR:1304906 (95m:14012)

[Mi 1] J. Milne, The points on a Shimura variety modulo a prime of good reduction, The zeta functions of Picard modular surfaces, 1992, pp. 151-253. MR1155229 (94g:11041) 
[Mi 2] J. Milne, Canonical models of (mixed) Shimura varieties and automorphic vector bundles, Automorphic forms, Shimura varieties. and $L$-functions I (Ann Arbor 1988), Perspectives in Math. 10, pp. 284-414, 1990. MR1044823 (91a:11027)

[Mi 3] J. Milne, On the conjecture of Langlands and Rapoport, available at www.arxiv.org (2007).

[Mi 4] J. Milne, Shimura varieties and motives, Motives (Seattle 1991), Proc. Sympos. Pure Math. 55, Part 2, Amer. Math. Soc., pp. 447-523, 1994. MR.1265562 (95c:11076)

[Mo] B. Moonen, Models of Shimura varieties in mixed characteristics, Galois representations in arithmetic algebraic geometry (Durham, 1996), London Math. Soc. Lecture Note Ser. 254, Cambridge Univ. Press, pp. 267-350, 1998. MR.1696489 (2000e:11077)

[PY] G. Prasad, J.-K. Yu, On quasi-reductive group schemes. With an appendix by Brian Conrad, J. Algebraic Geom. 15 (2006), 507-549. MR2219847(2007c:14047)

[RZ] M. Rapoport, T. Zink, Period space for p-divisible groups, Ann. Math. Studies 141, Princeton University Press, 1996. MR.1393439 (97f:14023)

[Sa] N. Saavedra Rivano, Catégories Tannakiennes, Lecture Notes in Math. 265, Springer, 1972. MR0338002 (49:2769)

[Sp] T. Springer, Reductive groups, Automorphic forms, representations and $L$-functions (Corvallis, 1977), Proc. Sympos. Pure Math. XXXIII, Amer. Math. Soc., pp. 1-27, 1979. MR.546587 (80h:20062)

[Ti] J. Tits, Reductive groups over local fields, Automorphic forms, representations and $L$ functions (Corvallis, 1977), Proc. Sympos. Pure Math. XXXIII, Amer. Math. Soc., pp. 2969, 1979. MR.546588 (80h:20064)

[Va 1] A. Vasiu, Integral Canonical Models of Shimura Varieties of Preabelian Type, Asian J. Math. 3 (1999), 401-518. MR1796512 (2002b:11087)

[Va 2] A. Vasiu, Integral Canonical Models of Shimura Varieties of Preabelian Type. (Fully corrected version.), available at www.arxiv.org (2003), 135 pages.

[Va 3] A. Vasiu, A motivic conjecture of Milne, available at www.arxiv.org (2003), 46 pages.

[Va 4] A. Vasiu, Good reductions of Shimura varieties of Hodge type in arbitrary unramified mixed characteristic I, available at www.arxiv.org (2007), 48 pages.

[Va 5] A. Vasiu, Good reductions of Shimura varieties of Hodge type in arbitrary unramified mixed characteristic II, available at www.arxiv.org (2007), 33 pages.

[Wa] W. Waterhouse, Introduction to affine group schemes, Graduate Texts in Mathematics, Springer, 1997. MR.547117 (82e:14003)

[Zi 1] T. Zink, The display of a formal p-divisible group, Cohomologies p-adiques et application arithmétiques I, Astérisque 278, Soc. Math. France, pp. 127-248, 2002. MR.1922825 (2004b:14083)

[Zi 2] T. Zink, Windows for displays of p-divisible groups, Moduli of abelian varieties (Text 1999), Prog. in Math. 195, Birkhäuser, pp. 491-518, 2001. MR.1827031(2002c:14073)

Department of Mathematics, University of Chicago, Chicago, Illinois 60637

Current address: Department of Mathematics, Harvard University, 1 Oxford Street, Cambridge, Massachusetts 02138

E-mail address: kisin@math.harvard.edu 